Comment. Math. Helv. 76 (2001) 218-262

(C) 2001 Birkhäuser Verlag, Basel

$0010-2571 / 01 / 020218-45 \$ 1.50+0.20 / 0$

Commentarii Mathematici Helvetici

\title{
Structures de contact sur les variétés fibrées en cercles au- dessus d'une surface
}

\author{
Emmanuel Giroux
}

Résumé. Soit $\mathrm{V}$ une variété fibrée en cercles au-dessus d'une surface $\mathrm{S}$ de genre $g>0$. Cet article fournit, pour les structures de contact sur $\mathrm{V}$, les analogues de résultats bien connus pour les feuilletages dûs à J. Milnor, J. Wood, W. Thurston, S. Matsumoto et É. Ghys. Dans la partie 1 , on démontre que $\mathrm{V}$ porte une structure de contact transversale aux fibres si et seulement si le nombre d'Euler de la fibration vaut au plus $2 g-2$. Dans la partie 2, on établit le fait suivant pour toute structure de contact $\xi$ sur $\mathrm{V}$ : ou bien $\xi$ est isotope à une structure transversale aux fibres, ou bien il existe, dans un revêtement fini de $\mathrm{V}$, une courbe legendrienne isotope aux fibres le long de laquelle $\xi$ définit la même trivialisation normale que la projection sur S. Dans la partie 3, on classifie les structures de contact transversales aux fibres à isotopie et conjugaison près. Dans la partie 4 , on étudie les structures de contact tendues quelconques sur $\mathrm{V}$; on montre que les structures virtuellement vrillées forment un nombre fini de classes d'isotopie tandis que les classes d'isotopie des structures universellement tendues sont en bijection avec les classes d'isotopie des multi-courbes essentielles sur S.

Mathematics Subject Classification (2000). 57M50, 57R17, 53D35, 53D10.

Mots-clés. Fibration en cercles, nombre d'Euler, structure de contact, tendue, vrillée.

Dans cet article, on essaie d'analyser le comportement global des structures de contact sur les variétés fibrées en cercles au-dessus d'une surface close. Plusieurs études antérieures motivent et guident ce travail. Tout d'abord, sur les fibrés principaux en cercles, les structures de contact invariantes admettent, à isotopie équivariante près, une classification remarquable $[\mathrm{Lu}]$. D'autre part, de nombreux résultats sur les représentations du groupe fondamental d'une surface dans $\mathrm{PSL}_{2}(\mathbf{R})$ et les homéomorphismes du cercle [EHN, Gh1, Gh2, Ma, Mi, Wo] contribuent à mettre à jour la structure topologique et dynamique des feuilletages de codimension 1 sur les variétés de dimension 3 fibrées en cercles (voir par exemple [Le, Th1, Th2]). Or, en dimension 3, les structures de contact sont, avec les fibrés tangents des feuilletages de codimension 1 , les seuls champs de plans localement homogènes et les développements parallèles des deux théories ont fait apparaître de nombreux traits communs.

Soit $\mathrm{V}$ une variété connexe et orientée, fibrée en cercles au-dessus d'une surface $\mathrm{S}$ close, orientable et de caractéristique d'Euler $\chi(\mathrm{S})$ négative ou nulle. 
Avant de présenter rapidement les principaux résultats de ce travail, on rappelle que $\mathrm{V}$, en tant que variété lisse orientée, est identifiée par le nombre d'Euler $\chi(\mathrm{V}, \mathrm{S})$ de la fibration $\pi: \mathrm{V} \rightarrow \mathrm{S}$ (cf. section 1.B). On rappelle aussi qu'une structure de contact (directe) sur $\mathrm{V}$ est un champ de plans défini localement comme le noyau d'une 1 -forme $\alpha$ dont le produit extérieur avec $d \alpha$ est une forme volume positive pour l'orientation choisie. Les plus grosses sous-variétés intégrales d'une structure de contact sont ainsi des courbes et portent le nom de courbes legendriennes.

Dans la partie 1, on démontre que $\mathrm{V}$ admet une « connexion strictement convexe », c'est-à-dire une structure de contact (directe) transversale aux fibres, si et seulement si le nombre d'Euler $\chi(\mathrm{V}, \mathrm{S})$ est inférieur ou égal à $-\chi(\mathrm{S})$ (théorème 1.1). Cette inégalité est un reliquat de l'inégalité de Milnor-Wood [Mi, Wo], laquelle peut en retour être interprétée comme suit : il existe sur $\mathrm{V}$ une connexion plate - i.e. un feuilletage transversal aux fibres - si et seulement si cohabitent sur $\mathrm{V}$ des structures de contact directes et indirectes transversales aux fibres.

Dans la partie 2, on donne une caractérisation géométrique des structures de contact qui sont isotopes à des connexions. Précisément, on prouve que toute structure de contact orientable $\xi$ sur $\mathrm{V}$ satisfait l'alternative exclusive suivante (théorème 2.3) : ou bien $\xi$ est isotope à une connexion, ou bien il existe, dans un revêtement fini de $(\mathrm{V}, \xi)$, une courbe legendrienne isotope à la fibre et le long de laquelle $\xi$ ne tourne pas, i.e. détermine la même trivialisation normale que la fibration $\pi$. Ce résultat est la version de contact d'un théorème démontré par W. Thurston dans [Th2] (voir aussi [Le]), selon lequel un feuilletage sur V est isotope à une connexion (plate) si et seulement si la fibre n'est pas isotope à une courbe tracée sur une feuille.

Dans la partie 3, on classifie les structures de contact transversales aux fibres à isotopie et conjugaison près (théorème 3.1 ). On montre en particulier que deux connexions strictement convexes peuvent ne pas être isotopes. La formule suivante résume bien la situation : il y a deux sortes de structures de contact transversales aux fibres, celles qui sont tangentes aux fibres et les autres. Ces dernières existent dès que $\chi(\mathrm{V}, \mathrm{S})<-\chi(\mathrm{S})$ et appartiennent à une même classe d'isotopie. Les premières, en revanche, n'existent que si $n \chi(\mathrm{V}, \mathrm{S})=-\chi(\mathrm{S})$ pour un certain entier $n \geq 1$ et leur classification se ramène à celle des revêtements fibrés à $n$ feuillets de $\mathrm{V}$ au-dessus de la variété $\mathbf{S}(\mathrm{TS})$ des droites orientées tangentes à $\mathrm{S}$; on montre ainsi que, pour $\chi(\mathrm{S})<0$, elles forment autant de classes de conjugaison qu'il y a de diviseurs de $n$.

Dans la partie 4, on tente de classer les structures de contact qui ne sont pas isotopes à des connexions. Comme le sort des structures de contact vrillées est scellé par un théorème de Y. Eliashberg [El1], on s'intéresse aux structures de contact tendues. Celles-ci présentent des comportements très différents selon qu'elles sont virtuellement vrillées ou universellement tendues (cf. définition 2.1). Les structures de contact virtuellement vrillées sur $\mathrm{V}$ constituent un nombre fini 
de classes d'isotopie (théorème 4.12) borné par $1+\sup \{0,-\chi(\mathrm{S})-\chi(\mathrm{V}, \mathrm{S})-1\}$. En revanche, les structures de contact universellement tendues et non isotopes à des connexions forment une infinité de classes d'isotopie qui sont en bijection naturelle avec les classes d'isotopie de systèmes (non vides) de courbes essentielles sur S (théorème 4.4). En fait, ces structures sont toutes isotopes à des structures de contact invariantes (par une quelconque action libre du cercle qui définit la fibration) et leur classification à isotopie près coïncide avec la classification des structures invariantes à isotopie équivariante près.

En parallèle avec ce dernier résultat, on établit une inégalité de Bennequin semi-locale (proposition 4.10) qui conduit à la classification des structures de contact tendues et $\mathbf{R}$-invariantes sur le produit par $\mathbf{R}$ de toute surface $F$ close et orientable (théorème 4.5) : les classes d'isotopie de ces structures sont à nouveau en bijection avec les classes d'isotopie de systèmes de courbes essentielles sur F . L'intérêt de ce résultat tient au fait qu'une surface $\mathrm{F}$ plongée dans une variété de contact de dimension 3 possède génériquement un voisinage tubulaire trivialisé $U \cong \mathrm{F} \times \mathbf{R}$ dans lequel la structure de contact est $\mathbf{R}$-invariante [Gi1].

Je tiens à remercier ici Étienne Ghys, Jean-Pierre Otal et Bruno Sévennec avec qui j'ai eu de nombreuses discussions sur certains aspects de ce travail. D'autre part, François Lalonde et Dietmar Salamon m'ont offert l'occasion de présenter les résultats discutés dans ce texte à Montréal en juin 1995 et à Warwick en mars 1998; je les en remercie vivement. Enfin, alors que cet article était déjà soumis, Ko Honda a annoncé des résultats très voisins.

\section{Existence de structures de contact transversales}

\section{A. Comment contacter Milnor-Wood}

Soit V une variété orientée, fibrée en cercles au-dessus d'une surface close S . Le théorème ci-dessous relate ce qui reste de l'inégalité de Milnor-Wood [Mi, Wo] quand on cherche non pas des feuilletages transversaux aux fibres - connexions plates - mais des structures de contact (directes) transversales aux fibres - connexions « strictement convexes ».

Théorème 1.1. Soit $\mathrm{V}$ une variété connexe orientée, fibrée en cercles au-dessus d'une surface close $\mathrm{S}$. Pour que $\mathrm{V}$ porte une structure de contact transversale aux fibres, il faut et il suffit que le nombre d'Euler $\chi(\mathrm{V}, \mathrm{S})$ de la fibration $\mathrm{V} \rightarrow \mathrm{S}$ vérifie l'inégalité

$$
\begin{cases}\chi(\mathrm{V}, \mathrm{S}) \leq-\chi(\mathrm{S}) & \text { si } \chi(\mathrm{S}) \leq 0 \\ \chi(\mathrm{V}, \mathrm{S})<0 & \text { si } \chi(\mathrm{S})>0\end{cases}
$$

Remarques.

a) La définition du nombre d'Euler $\chi(\mathrm{V}, \mathrm{S})$, et en particulier de son signe en 
fonction de l'orientation de $\mathrm{V}$, est rappelée dans la section B. Pour une variété $\mathrm{V}$ fibrée en cercles orientés au-dessus d'une surface $\mathrm{S}$ orientée, $\chi(\mathrm{V}, \mathrm{S})$ coïncide avec le nombre de Chern du fibré en droites complexes associé et l'orientation de $\mathrm{V}$ choisie est la juxtaposition des orientations de la base et de la fibre. Ainsi, la variété des droites orientées tangentes à $\mathrm{S}$ a pour nombre d'Euler $-\chi(\mathrm{S})$ lorsqu'elle est munie de l'orientation induite par sa structure de contact canonique.

b) Le théorème 1.1 a été indépendamment obtenu (du moins pour une surface $\mathrm{S}$ orientable) par A. Sato et T. Tsuboi [ST]. La preuve qu'on donne ci-après consiste simplement à adapter les arguments de J. Wood [Wo]. Par ailleurs, vu comme espace total d'un fibré principal en cercles, $\mathrm{V}$ admet des connexions invariantes qui sont des structures de contact directes si et seulement si $\chi(\mathrm{V}, \mathrm{S})$ est strictement négatif $[\mathrm{Lu}]$.

\section{B. Nombre d'Euler et connexions}

On rappelle d'abord ce qu'est le nombre d'Euler $\chi(\mathrm{V}, \mathrm{S})$ de la fibration $\pi: \mathrm{V} \rightarrow$ $\mathrm{S}$. On trace sur $\mathrm{S}$ un bouquet $\mathrm{K}$ de $2-\chi(\mathrm{S})$ cercles ayant pour complémentaire un disque et on note $\mathrm{D}$ (resp. $\mathrm{W}$ ) le disque polygonal (resp. le tore plein polyédral orienté) qu'on obtient en découpant $\mathrm{S}$ (resp. V) le long de $\mathrm{K}$ (resp. $\pi^{-1}(\mathrm{~K})$ ). Au-dessus de $\mathrm{K}$, la fibration $\pi$ admet des sections et chacune d'elles détermine, sur le bord orienté de $\mathrm{W}$, une courbe $\mathrm{C}$ dont la classe d'isotopie est invariable. De même, le bord des disques méridiens de $\mathrm{W}$ est une courbe $\mathrm{B}$ bien définie à isotopie près. Le nombre d'Euler $\chi(\mathrm{V}, \mathrm{S})$ est l'intersection homologique $\mathrm{B} \cdot \mathrm{C}$ de $\mathrm{B}$ et $\mathrm{C}$, ces deux courbes étant orientées de manière à couper les fibres dans le même sens.

Si S est une surface orientée, de genre $g$, et si V est munie d'une connexion $\xi$ (champ de plans transversal aux fibres), le nombre d'Euler $\chi(\mathrm{V}, \mathrm{S})$ s'interprète comme suit.

L'holonomie de $\xi$ associe à chaque cercle orienté $\mathrm{K}_{i}$ du bouquet $\mathrm{K}, 1 \leq$ $i \leq 2 g$, un difféomorphisme $\phi_{i}$ de la fibre $\mathbf{S}^{1}=\mathbf{R} / \mathbf{Z}$ qui surplombe le sommet de $\mathrm{K}$ : c'est l'application de premier retour qu'on obtient en suivant les courbes intégrales de $\xi$ au-dessus de $\mathrm{K}_{i}$. De plus, chaque classe d'homotopie de sections de $\left.\pi\right|_{\mathrm{K}_{i}}$ détermine un relèvement $\tilde{\phi}_{i}$ de $\phi_{i}$ à $\mathbf{R}$ : elle trivialise en effet $\left.\pi\right|_{\mathrm{K}_{i}}$ si bien que le segment de courbe intégrale qui joint $0 \in \mathbf{S}^{1}=\mathbf{R} / \mathbf{Z}$ à $\phi_{i}(0)$ se projette en un chemin sur la fibre $\mathbf{S}^{1}$; on prend alors pour $\tilde{\phi}_{i}(0)$ l'extrémité dans $\mathbf{R}$ du relevé partant de 0 .

De même, l'holonomie de la connexion induite par $\xi$ sur W, encore notée $\xi$, associe au bord orienté de $\mathrm{D}$ un difféomorphisme du cercle qui, à conjugaison près, s'écrit comme un mot

$$
\phi=w\left(\phi_{1}, \phi_{1}^{-1}, \ldots, \phi_{2 g}, \phi_{2 g}^{-1}\right)
$$

dans lequel chaque $\phi_{i}$, tout comme son inverse, intervient une fois et une seule - le 
découpage de $\mathrm{S}$ dédouble chaque cercle orienté $\mathrm{K}_{i}$ en deux arêtes de $\partial \mathrm{D}$ ayant des orientations incompatibles. Les courbes $\mathrm{B}$ et $\mathrm{C}$, respectivement fournies par les sections de $\pi$ au-dessus de $\mathrm{D}$ et de $\mathrm{K}$, déterminent alors deux relèvements distincts $\tilde{\phi}_{\mathrm{D}}$ et $\tilde{\phi}_{\mathrm{K}}$ de $\phi$ à $\mathbf{R}$. Compte tenu de ce qui précède, ceux-ci vérifient les identités suivantes :

- $\tilde{\phi}_{\mathrm{K}}=w\left(\tilde{\phi}_{1}, \tilde{\phi}_{1}^{-1}, \ldots, \tilde{\phi}_{2 g}, \tilde{\phi}_{2 g}^{-1}\right)$ où les $\tilde{\phi}_{i}$ sont des relèvements quelconques des $\phi_{i}$

- $\tilde{\phi}_{\mathrm{D}}(t)-\tilde{\phi}_{\mathrm{K}}(t)=\chi(\mathrm{V}, \mathrm{S})$ pour tout réel $t$.

\section{Inégalités clés}

Proposition 1.2. Soit $\xi$ une connexion sur $\mathrm{V}$ et $\tilde{\phi}_{\mathrm{K}}, \tilde{\phi}_{\mathrm{D}}$ les difféomorphismes de $\mathbf{R}$ définis plus haut.

a) Quelle que soit la connexion $\xi$,

$$
-2 g \leq \tilde{\phi}_{\mathrm{K}}(t)-t \leq 2 g \text { pour tout réel } t .
$$

b) Si $\xi$ est une structure de contact directe,

$$
\tilde{\phi}_{\mathrm{D}}(t)-t<0 \text { pour tout réel } t \text {. }
$$

Démonstration.

a) C'est l'inégalité de J. Wood [Wo]. Soit $\tilde{\mathcal{H}}$ l'espace des homéomorphismes de $\mathbf{R}$ qui commutent avec la translation $t \mapsto t+1$. L'application $h: \tilde{\mathcal{H}} \rightarrow \mathbf{R}$ définie par

$$
h(\tilde{\psi})=\sup \{\tilde{\psi}(t)-t, t \in \mathbf{R}\}
$$

vérifie

$$
h\left(\tilde{\psi}_{1} \tilde{\psi}_{2}\right) \leq h\left(\tilde{\psi}_{1}\right)+h\left(\tilde{\psi}_{2}\right) \leq h\left(\tilde{\psi}_{1} \tilde{\psi}_{2}\right)+1 \text { pour tous } \tilde{\psi}_{1}, \tilde{\psi}_{2} \in \tilde{\mathcal{H}} .
$$

On en déduit la majoration

$$
h\left(\tilde{\phi}_{\mathrm{K}}\right) \leq \sum_{i=1}^{2 g}\left(h\left(\tilde{\phi}_{i}\right)+h\left(\tilde{\phi}_{i}^{-1}\right)\right) \leq 2 g
$$

et on obtient la minoration en observant que

$$
\inf \{\tilde{\psi}(t)-t, t \in \mathbf{R}\}=-\sup \left\{\tilde{\psi}^{-1}(t)-t, t \in \mathbf{R}\right\} .
$$

b) Il s'agit de feuilleter $\mathrm{W}=\mathrm{D} \times \mathbf{S}^{1}$ par des disques méridiens dont le bord orienté soit partout transversal à $\xi$ et pointe du même côté que les fibres orientées. Par approximation, on peut supposer que $\mathrm{D}$ est un carré $[0,1]^{2}$. On choisit une coordonnée $\theta$ sur la fibre orientée en $(0,0)$ et on l'étend à $W$ en la décrétant constante sur chaque courbe intégrale de $\xi$ qui revêt soit un segment vertical soit 
la base du carré. Dans les coordonnées $(x, y, \theta) \in[0,1]^{2} \times \mathbf{S}^{1}$, la connexion $\xi$ a pour équation

$$
d \theta-u(x, y, \theta) d x=0 \quad \text { où } \quad u(x, 0, \theta)=0 \text { pour tout }(x, \theta) \in[0,1] \times \mathbf{S}^{1} .
$$

La condition qui exprime alors que $\xi$ est une structure de contact directe par rapport à $d x \wedge d y \wedge d \theta$ s'écrit $\partial_{y} u<0$. Les niveaux de $\theta$ sont ainsi des méridiens dont le bord orienté a la transversalité souhaitée le long du côté $y=1$ - car $u(x, 1, \theta)<0$ pour tout $(x, \theta)$ - et est tangent à $\xi$ ailleurs. On peut donc les perturber comme voulu.

Pour une surface $\mathrm{S}$ orientable et une connexion $\xi$ qui est une structure de contact directe, la proposition ci-dessus établit l'inégalité

$$
\chi(\mathrm{V}, \mathrm{S})<2 g=2-\chi(\mathrm{S}),
$$

qui n'est le résultat désiré que si $\mathrm{S}$ est la sphère. Si $\mathrm{S}$ est le plan projectif, le passage au revêtement double permet aussi de conclure. Dans les autres cas, l'inégalité du théorème 1.1 s'obtient via l'astuce classique suivante. On prend un revêtement à $n$ feuillets de $\mathrm{S}$ par une surface connexe orientable $\mathrm{S}_{n}$ et on note $\mathrm{V}_{n} \rightarrow \mathrm{S}_{n}$ le rappel du fibré $\mathrm{V} \rightarrow \mathrm{S}$ au-dessus de $\mathrm{S}_{n}$. Les relations

$$
\chi\left(\mathrm{S}_{n}\right)=n \chi(\mathrm{S}), \quad \chi\left(\mathrm{V}_{n}, \mathrm{~S}_{n}\right)=n \chi(\mathrm{V}, \mathrm{S}) \quad \text { et } \quad \chi\left(\mathrm{V}_{n}, \mathrm{~S}_{n}\right)<2-\chi\left(\mathrm{S}_{n}\right)
$$

donnent

$$
\chi(\mathrm{V}, \mathrm{S})<\frac{2}{n}-\chi(\mathrm{S}) .
$$

Comme on peut choisir $n$ arbitrairement grand, on obtient

$$
\chi(\mathrm{V}, \mathrm{S}) \leq-\chi(\mathrm{S}) .
$$

Remarque. Si la connexion $\xi$ est plate au sens où elle s'intègre en un feuilletage, le difféomorphisme $\tilde{\phi}_{\mathrm{D}}$ est l'identité et les arguments qui précèdent démontrent l'inégalité classique de Milnor-Wood, à savoir

$$
|\chi(\mathrm{V}, \mathrm{S})| \leq \sup \{0,-\chi(\mathrm{S})\}
$$

Suite au travail de S. Altschuler [Al], W. Thurston a inventé le terme de feuilletact (foliatact dans [Th4] muté en confoliation dans [ET]) pour désigner un champ de plans dont toute équation de Pfaff $\alpha$ est telle que la 3 -forme $\alpha \wedge d \alpha$ ne change pas de signe. La preuve de la proposition 1.2-b montre que, si la connexion $\xi$ est un feuilletact direct $(\alpha \wedge d \alpha \geq 0)$, le difféomorphisme $\tilde{\phi}_{\mathrm{D}}$ vérifie

$$
\tilde{\phi}_{\mathrm{D}}(t)-t \leq 0 \text { pour tout réel } t,
$$

de sorte que le nombre d'Euler $\chi(\mathrm{V}, \mathrm{S})$ satisfait à l'inégalité

$$
\chi(\mathrm{V}, \mathrm{S}) \leq \sup \{0,-\chi(\mathrm{S})\} .
$$




\section{Construction de structures transversales}

Lemme 1.3. Soit $\mathrm{V}$ et $\mathrm{V}^{\prime}$ deux variétés connexes et orientées, fibrées en cercles au-dessus d'une surface close $\mathrm{S}$. Si $\chi\left(\mathrm{V}^{\prime}, \mathrm{S}\right) \leq \chi(\mathrm{V}, \mathrm{S})$ et si $\mathrm{V}$ porte une structure de contact directe et transversale aux fibres, alors $\mathrm{V}^{\prime}$ en admet une aussi.

Démonstration. On suppose pour simplifier que la surface $\mathrm{S}$ et les fibres sont orientées. La définition du nombre d'Euler donnée dans la section B montre que $\mathrm{V}^{\prime}$ s'obtient à partir de $\mathrm{V}$ par la chirurgie suivante : on retire à $\mathrm{V}$ la préimage $\mathrm{W} \simeq \mathbf{D}^{2} \times \mathbf{S}^{1}$ d'un disque de $\mathrm{S}$ et on recolle un autre tore plein $\mathrm{W}^{\prime}$ par un difféomorphisme $\partial \mathrm{W}^{\prime} \rightarrow \partial \mathrm{W}$ qui respecte les fibres orientées et envoie le bord de chaque disque méridien de $\mathrm{W}^{\prime}$ sur une courbe de type $\left(1, \chi(\mathrm{V}, \mathrm{S})-\chi\left(\mathrm{V}^{\prime}, \mathrm{S}\right)\right)$ dans le produit $\partial \mathrm{W}=\partial \mathbf{D}^{2} \times \mathbf{S}^{1}$.

Si $\mathrm{V}$ porte une structure de contact $\xi$ directe et transversale aux fibres, la proposition 1.2-b montre qu'on peut feuilleter $\mathrm{W}$ par des disques méridiens dont le bord orienté soit transversal à $\xi$ et pointe du même côté que les fibres orientées. Si $\chi\left(\mathrm{V}^{\prime}, \mathrm{S}\right) \leq \chi(\mathrm{V}, \mathrm{S})$, un tel feuilletage par disques méridiens existe aussi sur $\mathrm{W}^{\prime}$ et il est alors facile de prolonger à $\mathrm{W}^{\prime}$ la structure $\left.\xi\right|_{\mathrm{V} \backslash \mathrm{W}}$ pour obtenir sur $\mathrm{V}^{\prime}$ une structure de contact directe et transversale aux fibres.

Pour chaque surface $\mathrm{S}$, il reste donc à construire une structure de contact directe et transversale aux fibres sur la variété orientée $\mathrm{V}$ dont le nombre d'Euler $\chi(\mathrm{V}, \mathrm{S})$ est le plus grand autorisé par l'inégalité du théorème 1.1.

Pour la sphère $\mathbf{S}^{2}$, la structure de contact usuelle sur $\mathbf{S}^{3}$ est orthogonale aux cercles de Hopf et fournit l'exemple voulu. Pour toutes les autres surfaces (y compris $\mathbf{P}^{2}$ ), la variété $\mathrm{V}$ est celle des droites orientées tangentes à $\mathrm{S}$. Elle porte une structure de contact canonique $\xi_{\mathrm{S}}$ qui induit l'orientation pour laquelle $\chi(\mathrm{V}, \mathrm{S})=-\chi(\mathrm{S})$ mais qui est tangente aux fibres et non pas transversale. En effet, si $\delta$ est une droite orientée tangente à $\mathrm{S}$ en un point $q$, le plan $\xi_{\mathrm{S}}(q, \delta) \subset \mathrm{T}_{(q, \delta)} \mathrm{V}$ est l'image inverse de $\delta$ par la projection. En choisissant dans $\xi_{\mathrm{S}}(q, \delta)$, pour tout $(q, \delta)$, un vecteur non vertical dont la projection sur $\mathrm{S}$ donne l'orientation de $\delta$, on fabrique sur $\mathrm{V}$ un champ de vecteurs legendrien non singulier et transversal aux fibres. La condition de contact assure que, si on pousse $\xi_{\mathrm{S}}$ par le flot de ce champ pendant un bref instant, on obtient une structure transversale aux fibres. Cet argument démontre plus généralement le fait suivant :

Proposition 1.4. Soit V une variété connexe et orientée, fibrée en cercles audessus d'une surface orientable $\mathrm{S}$. Toute structure de contact tangente aux fibres et orientable le long des fibres est déformable en une structure de contact transversale aux fibres par une isotopie arbitrairement petite. 


\section{E. Autre construction par la géométrie hyperbolique}

Pour clore cette partie, voici une autre construction adaptée de la théorie des feuilletages. Soit $\mathrm{S}$ une surface close orientable, de genre $g \geq 1$, et $\mathrm{V}$ une variété connexe orientée fibrée en cercles au-dessus de $\mathrm{S}$. Si on prend sur $\mathrm{S}$ un bouquet de cercles $\mathrm{K}=\bigvee_{i=1}^{k} \mathrm{~K}_{i}$, de sommet $q$, et si on se donne $k$ difféomorphismes $\phi_{i}$ de la fibre $\mathbf{S}^{1}=\pi^{-1}(q)$, on peut fabriquer près de $\pi^{-1}(\mathrm{~K})$ une structure de contact transversale aux fibres dont l'holonomie au-dessus de chaque cercle $\mathrm{K}_{i}$ vaille $\phi_{i}$. En fait, toute structure de contact transversale aux fibres définie sur un petit voisinage de $\pi^{-1}(q)$ admet un tel prolongement. Ainsi, vu la discussion des sections $\mathrm{B}$ et $\mathrm{C}$, construire sur $\mathrm{V}$ une structure de contact transversale aux fibres revient à trouver $2 g$ difféomorphismes $\phi_{i}$ du cercle dont les relèvements $\tilde{\phi}_{i}$ à $\mathbf{R}$ vérifient

$$
\left(\prod_{i=1}^{g}\left[\tilde{\phi}_{2 i-1}, \tilde{\phi}_{2 i}\right]\right)(t)-t<-\chi(\mathrm{V}, \mathrm{S}) \quad \text { pour tout réel } t .
$$

L'argument ci-dessous, soufflé par É. Ghys et tout empreint de [Th1] (voir aussi $[\mathrm{EHN}]$ ), fournit $2 g$ éléments $\phi_{i}$ de $\mathrm{PSL}_{2}(\mathbf{R})$ dont les relèvements $\widetilde{\phi}_{i}$ vérifient

$$
\left(\prod_{i=1}^{g}\left[\tilde{\phi}_{2 i-1}, \tilde{\phi}_{2 i}\right]\right)(t)-t<\chi(\mathrm{S}) \quad \text { pour tout réel } t .
$$

Soit $\mathrm{P}$ un polygone convexe à $4 g$ côtés dans le plan hyperbolique $\mathbf{H}^{2}$. On suppose que les sommets de $\mathrm{P}$, numérotés $s_{1}, \ldots, s_{4 g}$ dans le sens des aiguilles d'une montre, vérifient

$$
\left\{\begin{array}{l}
\operatorname{dist}\left(s_{4 i-3}, s_{4 i-2}\right)=\operatorname{dist}\left(s_{4 i-1}, s_{4 i}\right) \\
\operatorname{dist}\left(s_{4 i-2}, s_{4 i-1}\right)=\operatorname{dist}\left(s_{4 i}, s_{4 i+1}\right)
\end{array} \quad \text { pour } 1 \leq i \leq g \text { et } s_{4 g+1}=s_{1} .\right.
$$

On colle alors isométriquement chaque arête orientée $\left[s_{4 i-3}, s_{4 i-2}\right]$ (resp. $\left.\left[s_{4 i-2}, s_{4 i-1}\right]\right)$ sur l'arête orientée $\left[s_{4 i}, s_{4 i-1}\right]$ (resp. $\left.\left[s_{4 i+1}, s_{4 i}\right]\right)$. On obtient ainsi une surface close orientable $\mathrm{S}$ de genre $g$ munie d'une métrique hyperbolique ayant une singularité conique en $s$, point image des sommets de $\mathrm{P}$. On pose ensuite $\mathrm{R}=\mathrm{S} \backslash\{s\}$, on choisit dans $\mathrm{R}$ un point de référence $r$, image d'un point $r_{*} \in \operatorname{Int} \mathrm{P}$ situé très près de $s_{1}$, et on note $(\tilde{\mathrm{R}}, \tilde{r})$ le revêtement universel de $(\mathrm{R}, r)$. Ces données déterminent une application développante $\mathrm{D}:(\tilde{\mathrm{R}}, \tilde{r}) \rightarrow$ $\left(\mathbf{H}^{2}, r_{*}\right)$ et une représentation d'holonomie $h: \pi_{1}(\mathrm{R}) \rightarrow \mathrm{PSL}_{2}(\mathbf{R})$.

Soit $\mathrm{C} \subset \mathrm{S}$ le cercle trigonométrique de centre $s$ passant par $r$ et $\gamma \in \pi_{1}(\mathrm{~S})$ sa classe d'homotopie. Le point $\mathrm{D}(\gamma \cdot \tilde{r})=h(\gamma)\left(r_{*}\right)$ est l'image de $r_{*}$ par la rotation hyperbolique de centre $s_{1}$ et d'angle la somme des angles intérieurs de $\mathrm{P}$, à savoir $(4 g-2) \pi-\operatorname{aire}(\mathrm{P})$ d'après la formule de Gauss-Bonnet. D'autre part, $h(\gamma)$ est le produit de $g$ commutateurs dans $\mathrm{PSL}_{2}(\mathbf{R})$. Pour les identifier, on note que l'image inverse de $\mathrm{C}$ dans $\mathrm{P}$ est formée de $4 g$ arcs de cercles qui, en partant de $r_{*}$, sont centrés successivement aux points $s_{1}, s_{4}, s_{3}, s_{2}, s_{5}, s_{8}, s_{7}, s_{6}, \ldots$. Soit 
alors $\phi_{2 i-1}$ et $\phi_{2 i}, 1 \leq i \leq g$, les éléments de $\operatorname{PSL}_{2}(\mathbf{R})$ caractérisés par les propriétés suivantes :

$$
\begin{aligned}
\phi_{2 i-1}\left(s_{4 i-1}\right) & =s_{4 i-2} & & \phi_{2 i}\left(s_{4 i-2}\right)=s_{4 i+1} \\
\phi_{2 i-1}\left(s_{4 i}\right) & =s_{4 i-3} & \phi_{2 i}\left(s_{4 i-3}\right) & =s_{4 i} .
\end{aligned}
$$

Par construction, $\prod_{i=1}^{g}\left[\phi_{2 i-1}, \phi_{2 i}\right]$ vaut bien $h(\gamma)$. Reste à déterminer le nombre de translation du produit des commutateurs des relèvements $\tilde{\phi}_{i}$. Pour cela, on regarde le cas limite où $\mathrm{P}$ est un polygone euclidien dans le plan tangent à $\mathbf{H}^{2}$ en un point $s_{0}$. Dans ce cas, les transformations $\phi_{i}$ sont toutes des rotations de centre $s_{0}$ et commutent donc. Par suite, $\prod_{i=1}^{g}\left[\tilde{\phi}_{2 i-1}, \tilde{\phi}_{i}\right]=\mathrm{id}$. Il en résulte que, dans le cas général, le nombre de translation vaut $-\frac{1}{2 \pi}$ aire $(\mathrm{P})$ et prend ainsi n'importe quelle valeur entre 0 et $(1-2 g)$. En particulier, pour obtenir une structure de contact sur le fibré $\mathbf{S}(\mathrm{TS})$ des droites orientées tangentes à $\mathrm{S}$, il faut partir d'un polygone d'aire supérieure à $(4 g-4) \pi$, i.e. d'une métrique ayant un atome de courbure positive en la singularité conique.

\section{Caractérisation des structures de contact transversales}

\section{A. Comment contacter Thurston}

V désigne toujours une variété connexe orientée, fibrée en cercles au-dessus d'une surface close S. Dans [Th2], W. Thurston met en évidence l'alternative exclusive suivante : si $\xi$ est un feuilletage de codimension 1 orientable sur $\mathrm{V}$, ou bien $\xi$ est isotope à un feuilletage transversal aux fibres, ou bien il existe une courbe simple tracée sur une feuille qui est isotope à la fibre. Dans le second cas, $\xi$ possède en fait un ensemble minimal vertical - à isotopie près - qui, pour peu que $\xi$ soit $\mathcal{C}^{2}$ et que $\mathrm{S}$ ne soit pas un tore, est nécessairement une feuille torique. Le résultat qui suit est un analogue de ce théorème pour les structures de contact. Son énoncé requiert un peu de terminologie.

Définition 2.1. Soit $\xi$ une structure de contact sur une variété $M$ de dimension 3 . On dit que $\xi$ est vrillée s'il existe un disque $\mathrm{D}$ plongé dans $\mathrm{M}$ qui est tangent à $\xi$ en tous les points de son bord - le disque D est lui-même appelé disque vrillé. On dit que $\xi$ est tendue si elle n'est pas vrillée.

Une structure vrillée sur M induit une structure encore vrillée sur tout revêtement de $M$ mais une structure tendue peut aussi induire une structure vrillée sur certains revêtements. On dira qu'une structure de contact $\xi$ sur M est virtuellement vrillée (resp. universellement tendue) si elle est tendue et si elle induit une structure vrillée (resp. tendue) sur un revêtement fini de M (resp. sur le revêtement universel de M ). Ces deux propriétés s'excluent mutuellement mais il n'est pas clair qu'elles soient exactement complémentaires l'une de l'autre. C'est cependant le cas si le groupe fondamental de $\mathrm{M}$ est résiduellement fini, donc par exemple pour $\mathrm{V}$. 
Le long d'une fibre de $\mathrm{V}$, les champs de vecteurs normaux dont la projection sur $\mathrm{S}$ est constante déterminent une classe d'homotopie canonique de sections non singulières du fibré normal. De plus, dès que $\mathrm{V}$ diffère de $\mathbf{S}^{2} \times \mathbf{S}^{1}$, cette classe d'homotopie est invariante par tout difféomorphisme de $\mathrm{V}$ isotope à l'identité qui préserve la fibre considérée. Du coup, le fibré normal de toute courbe fermée simple isotope à la fibre possède aussi une classe d'homotopie canonique de sections partout non nulles. Par abus de langage, lorsque $\mathrm{V} \simeq \mathbf{S}^{2} \times \mathbf{S}^{1}$, une courbe isotope à la fibre désigne dans la suite une courbe munie d'une isotopie qui l'amène sur une fibre.

Définition 2.2. Soit $\xi$ une structure de contact sur V. Pour toute courbe legendrienne $\mathrm{L}$ isotope à la fibre, on appelle enroulement de $\xi$ autour de $\mathrm{L}$ - ou enroulement de $\mathrm{L}-$ le nombre e $(\mathrm{L})$ de tours que $\xi$ fait le long de $\mathrm{L}$ par rapport au champ normal canonique. On appelle enroulement de $\xi$ et on note e $(\xi)$ le supremum des enroulements $\mathrm{e}(\mathrm{L})$ pour toutes les courbes legendriennes $\mathrm{L}$ isotopes aux fibres. Ce nombre est un entier si et seulement si la structure de contact $\xi$ est orientable le long des fibres.

Remarque. Dès lors que $\mathrm{V}$ n'est ni un espace lenticulaire ni un tore, un théorème de F. Waldhausen [Wa] assure que tous les difféomorphismes de $\mathrm{V}$ respectent la fibration $\pi: \mathrm{V} \rightarrow \mathrm{S}$, à isotopie près. L'enroulement des structures de contact sur $\mathrm{V}$ est alors invariant par conjugaison.

Enfin, l'énoncé ci-dessous tient tacitement compte du fait que tout revêtement fini de $\mathrm{V}$ fibre naturellement en cercles au-dessus d'un revêtement fini de $\mathrm{S}$.

Théorème 2.3. Soit V une variété connexe et orientée, fibrée en cercles audessus d'une surface close et orientable $\mathrm{S}$. Toute structure de contact $\xi$ sur $\mathrm{V}$ qui est orientable le long des fibres vérifie l'alternative exclusive suivante:

- ou bien $\xi$ est isotope à une structure de contact transversale aux fibres;

- ou bien il existe, dans un revêtement fini $(\tilde{\mathrm{V}}, \tilde{\xi})$ de $(\mathrm{V}, \xi)$, une courbe legendrienne isotope à la fibre de $\tilde{\mathrm{V}}$ et d'enroulement nul. Mieux, le passage à un revêtement fini n'est nécessaire que lorsque $\xi$ est virtuellement vrillée.

Ce théorème est une conséquence des deux propositions ci-dessous dont la démonstration occupe la suite de la partie 2 .

Proposition 2.4. Soit $\xi$ une structure de contact sur $\mathrm{V}$ orientable le long des fibres.

a) L'enroulement $\mathrm{e}(\xi)$ appartient à $\mathbf{Z} \cup\{+\infty\}$. En outre, pour tout entier $n \leq \mathrm{e}(\xi)$, il existe dans $\mathrm{V}$ une courbe legendrienne $\mathrm{L}$ isotope à la fibre et d'enroulement $\mathrm{e}(\mathrm{L})$ égal à $n$.

b) Si $\xi$ est vrillée, son enroulement $\mathrm{e}(\xi)$ est infini.

c) Si $\xi$ est transversale aux fibres, $\xi$ est universellement tendue et son enroulement $\mathrm{e}(\xi)$ est strictement négatif. 
Proposition 2.5. Soit $\xi$ une structure de contact sur $\mathrm{V}$ orientable le long des fibres. Si $\xi$ est universellement tendue et si son enroulement e $(\xi)$ est strictement négatif, $\xi$ est isotope à une structure de contact transversale aux fibres.

Démonstration du théorème 2.3. Soit $\xi$ une structure de contact sur V orientable le long des fibres. La proposition 2.4 montre tout d'abord que l'alternative envisagée pour $\xi$ est exclusive. Elle assure aussi que, si $\xi$ est vrillée (resp. virtuellement vrillée), il existe dans $\mathrm{V}$ (resp. dans un revêtement fini de $\mathrm{V}$ ) une courbe legendrienne isotope à la fibre et d'enroulement nul. Si $\xi$ est au contraire universellement tendue, de deux choses l'une : ou bien son enroulement e $(\xi)$ est strictement négatif et $\xi$ est isotope à une structure transversale aux fibres (proposition 2.5), ou bien $\mathrm{e}(\xi)$ est positif ou nul et il existe dans $\mathrm{V}$ une courbe legendrienne isotope à la fibre et d'enroulement nul (proposition 2.4).

\section{B. Estimations d'enroulement}

On démontre ici la proposition 2.4. Pour cela, on rappelle que l'invariant de Thurston-Bennequin $\mathrm{tb}(\mathrm{L})$ d'une courbe legendrienne $\mathrm{L}$ homologiquement nulle dans $(\mathrm{V}, \xi)$ est l'enlacement de $\mathrm{L}$ avec $\mathrm{L}+\nu$, où $\nu$ est un champ de vecteurs normal à $\xi$ le long de $\mathrm{L}$. L'enroulement est un cousin de cet invariant; en particulier, si $\chi(\mathrm{V}, \mathrm{S})= \pm 1$, toute courbe legendrienne L isotope à la fibre est homologiquement nulle et $\mathrm{e}(\mathrm{L})=\mathrm{tb}(\mathrm{L}) \pm 1$.

a) Comme $\xi$ est orientable le long des fibres, elle effectue un nombre entier de tours autour de chaque courbe legendrienne isotope à la fibre. ${ }^{1}$ Par suite, e $(\xi) \in$ $\mathbf{Z} \cup\{+\infty\}$. Soit maintenant $\mathrm{L}_{0}$ une courbe legendrienne isotope à la fibre et d'enroulement $\mathrm{e}\left(\mathrm{L}_{0}\right) \geq n, n \in \mathbf{Z}$. Soit d'autre part $\mathrm{L}_{1}$ un nœud legendrien topologiquement trivial contenu dans une boule disjointe de $\mathrm{L}_{0}$ et dont l'invariant de Thurston-Bennequin vaut $\operatorname{tb}\left(\mathrm{L}_{1}\right)=n-\mathrm{e}\left(\mathrm{L}_{0}\right)-1 \leq-1$. La somme connexe de $\mathrm{L}_{0}$ et $\mathrm{L}_{1}$ est une courbe legendrienne isotope à la fibre et d'enroulement $n$.

b) Soit $\mathrm{B} \subset \mathrm{V}$ une boule contenant un disque vrillé de $\xi$ et soit $\mathrm{L}_{0}$ une courbe legendrienne isotope à la fibre et disjointe de $\mathrm{B}$. Pour tout $n \geq 0$, il existe dans B un nœud legendrien topologiquement trivial dont l'invariant de ThurstonBennequin vaut $n$. La somme connexe de $\mathrm{L}_{0}$ avec ce nœud fournit une courbe legendrienne isotope à la fibre dont l'enroulement vaut $\mathrm{e}\left(\mathrm{L}_{0}\right)+n+1$. Par suite, $\mathrm{e}(\xi)=+\infty$.

c) Soit $\tilde{\xi}$ la structure induite par $\xi$ sur le revêtement universel $\tilde{\mathrm{V}}$ de V. Si S n'est pas une sphère, $\tilde{V}$ est difféomorphe à $\mathbf{R}^{3}$. Ainsi, à conjugaison près, $\tilde{\xi}$ est une structure de contact sur $\mathbf{R}^{2} \times \mathbf{R}$ transversale aux droites $\{*\} \times \mathbf{R}$ et invariante par les translations verticales entières. Comme pour la proposition 1.2-b, on

\footnotetext{
1 On observe au passage qu'une structure de contact transversale aux fibres est automatiquement (co) orientable le long des fibres.
} 
construit sur tout domaine $[-a-1, a+1]^{2} \times \mathbf{R}, a>0$, des coordonnées $(x, y, t)$ dans lesquelles la fibration est la projection $(x, y, t) \mapsto(x, y)$ et $\tilde{\xi}$ a pour équation $d t-u(x, y, t) d x=0$, où $u(x,-a, t)=0$ et $u(x, y, t+1)=u(x, y, t)$ quels que soient $(x, y, t)$.

Pour tout entier $n>0$, l'immersion $\phi_{n}:[-a, a]^{2} \times \mathbf{R} \rightarrow \mathbf{R}^{3}$, définie - en coordonnées cylindriques au but - par

$$
(x, y, t) \longmapsto\left(r=u(x, y, t)^{-1 / 2}, \theta=\frac{2 \pi t}{n}, z=\frac{2 \pi x}{n}\right),
$$

plonge $[-a, a]^{2} \times \mathbf{R} / n \mathbf{Z}$ dans $\mathbf{R}^{3}$ privé de l'axe des $z$ et envoie $\tilde{\xi}$ sur la structure d'équation $d z=r^{2} d \theta$. Par suite, $\tilde{\xi}$ est tendue en vertu du théorème de Bennequin.

Soit maintenant $\mathrm{L}$ une courbe legendrienne isotope à la fibre et $\tilde{\mathrm{L}}$ une préimage de $\mathrm{L}$ dans $\mathbf{R}^{2} \times \mathbf{R} / \mathbf{Z}$. Étant donné $a>0$ assez grand pour que $[-a, a]^{2} \times \mathbf{R} / \mathbf{Z}$ contienne $\tilde{\mathbf{L}}$, le plongement induit par $\phi_{1}$ sur $[-a, a]^{2} \times \mathbf{R} / \mathbf{Z}$ envoie les fibres $\{*\} \times \mathbf{R} / \mathbf{Z}$ sur des courbes deux à deux non enlacées mais qui enlacent une fois l'axe des $z$. La courbe legendrienne $\phi_{1}(\tilde{\mathrm{L}})$ est alors non nouée et son invariant de Thurston-Bennequin n'est autre que e( $(\tilde{\mathrm{L}})$. Il résulte donc de l'inégalité de Bennequin $[\mathrm{Be}]$ que $\mathrm{e}(\tilde{\mathrm{L}})=\mathrm{e}(\mathrm{L}) \leq-1$.

Si $\mathbf{S}$ est une sphère, $\tilde{V}$ est difféomorphe à la sphère $\mathbf{S}^{3}$ car $\mathbf{S}^{2} \times \mathbf{S}^{1}$ ne porte aucune structure de contact transversale aux fibres (théorème 1.1). Ainsi, à conjugaison près, $\tilde{\xi}$ est une structure de contact sur $\mathbf{S}^{3}$ transversale à la fibration de Hopf. Si on regarde $\mathbf{S}^{3}$ comme le bord de la boule unité dans $\mathbf{C}^{2}$ - les cercles de Hopf étant les traces des droites complexes passant par $\underset{\tilde{\xi}}{0}$-, la forme symplectique usuelle de $\mathbf{C}^{2}$ est positive sur $\tilde{\xi}$, ce qui entraîne que $\tilde{\xi}$ est tendue [El3].

Soit enfin $\mathrm{L}$ une courbe legendrienne isotope à la fibre et $\tilde{\mathrm{L}}$ une préimage de $\mathrm{L}$ dans $\tilde{\mathrm{V}} \simeq \mathbf{S}^{3}$. La courbe $\tilde{\mathrm{L}}$ est non nouée et, comme $\tilde{\xi}$ est tendue, l'inégalité de Bennequin assure que $\operatorname{tb}(\tilde{\mathrm{L}}) \leq-1$, donc que e $(\tilde{\mathrm{L}})=\operatorname{tb}(\mathrm{L})-1 \leq-2$. Or $\mathrm{e}(\tilde{\mathrm{L}})=|\chi(\mathrm{V}, \mathrm{S})| \mathrm{e}(\mathrm{L})$, donc $\mathrm{e}(\mathrm{L})$ est strictement négatif.

\section{Le cas des fibrés sur la sphère}

On démontre ici la proposition 2.5 lorsque $\mathrm{S}$ est une sphère. L'ingrédient clé est la classification des structures de contact tendues sur les espaces lenticulaires [Gi4, théorème 1.1]. Le point qui intervient ici est le suivant : tout espace lenticulaire porte une seule structure de contact universellement tendue, à isotopie près. ${ }^{2}$

$\mathrm{Si} \chi(\mathrm{V}, \mathrm{S})=0$, la variété $\mathrm{V}$ est difféomorphe à $\mathbf{S}^{2} \times \mathbf{S}^{1}$. Or, d'après [El2], $\mathbf{S}^{2} \times \mathbf{S}^{1}$ porte une unique structure de contact tendue qu'on peut voir par exemple comme le champ $\xi_{0}$ des droites complexes tangentes au bord de $X_{\varepsilon}$, où $X_{\varepsilon} \subset \mathbf{C}^{2}$ est le tube de rayon $\varepsilon<1$ autour du cercle unité $\mathbf{S}^{1} \times\{0\}$. On observe alors que le tore $\mathrm{T}=\mathbf{S}^{1} \times \varepsilon \mathbf{S}^{1}$ est contenu dans $\partial X_{\varepsilon}$ et que $\xi_{0}$ est parallèle à $\mathbf{C} \times\{0\}$

$\overline{2}$ Il s'agit ici de structures de contact orientables mais pas orientées. 
le long de $\mathrm{T}$. Par suite, chaque cercle $\mathbf{S}^{1} \times\{w\}, w \in \varepsilon \mathbf{S}^{1}$, est legendrien et d'enroulement nul. Aucune structure de contact tendue sur V n'a donc un enroulement strictement négatif.

Si $\chi(\mathrm{V}, \mathrm{S}) \neq 0$, le revêtement universel de $\mathrm{V}$ est difféomorphe à $\mathrm{S}^{3}$ et on peut voir $\mathrm{V}$ comme suit. On regarde $\mathbf{S}^{3}$ comme le bord orienté de la boule unité dans $\mathbf{C}^{2}$ et, pour tout $t \in \mathbf{R} / \mathbf{Z}$, on note $\phi_{t}^{+}$et $\phi_{t}^{-}$les transformations de Hopf définies par

$$
\left\{\begin{array}{l}
\phi_{t}^{+}(z, w)=\left(e^{2 i \pi t} z, e^{+2 i \pi t} w\right), \\
\phi_{t}^{-}(z, w)=\left(e^{2 i \pi t} z, e^{-2 i \pi t} w\right), \quad(z, w) \in \mathbf{S}^{3} .
\end{array}\right.
$$

La fibration $\mathbf{S}^{3} \rightarrow \mathbf{S}^{2}$ associée au flot $\phi_{t}^{+}$(resp. $\phi_{t}^{-}$) a pour nombre d'Euler -1 (resp. +1 ). Pour tout entier $n>0$, le quotient de $\mathbf{S}^{3}$ par $\phi_{1 / n}^{+}$(resp. $\left.\phi_{1 / n}^{-}\right)$a donc pour nombre d'Euler $-n$ (resp. $n$ ). En outre, ce quotient n'est autre que l'espace lenticulaire $\mathbf{L}_{n, 1}\left(\operatorname{resp} . \quad \mathbf{L}_{n, n-1}\right)$ et est difféomorphe à $\mathrm{V}$ si $\chi(\mathrm{V}, \mathrm{S})=-n($ resp. si $\chi(\mathrm{V}, \mathrm{S})=n)$.

Soit maintenant $\xi_{0}$ la structure de contact usuelle sur $\mathbf{S}^{3}$, i.e. le champ des droites complexes tangentes à $\mathbf{S}^{3}$. Comme chaque transformation $\phi_{t}^{ \pm}$est la restriction d'une application linéaire de $\mathbf{C}^{2}$, elle préserve $\xi_{0}$. Ainsi, $\xi_{0}$ induit sur chacun des espaces $\mathbf{L}_{n, 1}$ et $\mathbf{L}_{n, n-1}$ une structure de contact $\xi$ qui est universellement tendue (car $\xi_{0}$ est tendue d'après le théorème de Bennequin). D'autre part, les orbites du flot $\phi_{t}^{+}$sont transversales à $\xi_{0}$ de sorte que $\xi$ est transversale aux fibres sur $\mathbf{L}_{n, 1}$. En revanche, sur le tore invariant

$$
\left\{(z, w) \in \mathbf{S}^{3}|| z|=| w \mid\right\},
$$

les orbites du flot $\phi_{t}^{-}$sont tangentes à $\xi_{0}$ et d'enroulement nul. Par suite, $\xi$ n'a pas un enroulement strictement négatif. Ces observations terminent la démonstration puisque $\mathbf{L}_{n, 1}$ et $\mathbf{L}_{n, n-1}$ portent chacun une unique structure de contact universellement tendue, à isotopie près [Gi4, théorème 1.1 et lemme 4.1].

\section{Surfaces convexes}

On introduit ici quelques notions et résultats techniques qui seront utiles dans les démonstrations à venir.

Définition 2.6. Soit $F$ une surface, orientable et compacte, plongée dans une variété de contact $(\mathrm{M}, \xi)$ de dimension 3 . On dit que $\mathrm{F}$ est convexe si elle admet un voisinage tubulaire trivialisé $U \simeq \mathrm{F} \times \mathbf{R}$ dans lequel les translations verticales préservent $\xi$. Un tel voisinage $U$ sera dit homogène.

La convexité de $\mathrm{F}$ ne dépend que du germe de $\xi$ le long de $\mathrm{F}$, donc du feuilletage caractéristique $\xi \mathrm{F}$ de $\mathrm{F}$ formé des courbes intégrales du champ de droites $\xi \cap \mathrm{TF}$. Si F est close, elle se traduit explicitement comme suit. On dit 
qu'une multi-courbe $\Gamma \subset \mathrm{F}$ - union finie de courbes fermées, simples et disjointes scinde $\xi \mathrm{F}$ si, sur la surface compacte à bord $\mathrm{F}_{\Gamma}$ obtenue en découpant $\mathrm{F}$ le long de $\Gamma$, le feuilletage induit par $\xi \mathrm{F}$ est porté par un champ de vecteurs qui sort sur $\partial \mathrm{F}_{\Gamma}$ et qui dilate l'aire (i.e. une certaine forme d'aire sur $\mathrm{F}_{\Gamma}$ ). Lorsqu'une telle multi-courbe existe, elle est unique à isotopie près parmi les multi-courbes qui scindent.

Les résultats de [Gi1] montrent qu'une surface close $\mathrm{F} \subset(\mathrm{M}, \xi)$ est convexe si et seulement si son feuilletage $\xi \mathrm{F}$ est scindé. En particulier, si $U$ est un voisinage tubulaire et homogène d'une surface convexe $\mathrm{F}$, l'ensemble noté $\Gamma_{U}$ des points de $\mathrm{F}$ où $\xi$ est tangente aux fibres de $U$ est une multi-courbe qui scinde $\xi \mathrm{F}$.

Cette caractérisation permet de montrer que les surfaces closes convexes sont génériques. En outre, elles sont très maniables et certaines ont un réel intérêt géométrique :

Exemple. Suivant une suggestion de V. I. Arnold, on appellera surface clairaldienne toute surface compacte convexe $\mathrm{F} \subset(\mathrm{M}, \xi)$ qui est munie d'une fibration en cercles legendriens, au-dessus de l'intervalle ou du cercle. Topologiquement, une telle surface est donc un anneau, un tore ou une bouteille de Klein.

Si $\pi: \mathrm{V}_{0} \rightarrow \mathrm{S}_{0}$ est une fibration legendrienne, l'image inverse $\pi^{-1}(\mathrm{C})$ de toute courbe simple $\mathrm{C} \subset \mathrm{S}_{0}$ (fermée ou non) est une surface clairaldienne. En effet, tout flot local transversal à $\mathrm{C}$ dans $\mathrm{S}_{0}$ se relève naturellement dans $\mathrm{V}_{0}$ en un flot de contact transversal à $\pi^{-1}(\mathrm{C})$. Inversement, toute surface clairaldienne $\mathrm{F} \subset$ $(\mathrm{M}, \xi)$ est localement de ce type $\pi^{-1}(\mathrm{C})$, où $\mathrm{V}_{0}$ est un voisinage homogène quelconque de $\mathrm{F}$ et $\mathrm{S}_{0}$ un fibré en intervalles au-dessus de $\mathrm{C}$. Ainsi, toute surface clairaldienne orientable - difféomorphe à $\mathbf{S}^{1} \times \mathrm{C}$ où $\mathrm{C}$ est l'intervalle ou le cercle - possède un voisinage tubulaire homogène $U \simeq \mathbf{S}^{1} \times \mathbf{C} \times \mathbf{R}$ dans lequel $\mathrm{F}=\mathbf{S}^{1} \times \mathrm{C} \times\{0\}$ et $\xi$ a une équation de la forme

$$
\cos (n \pi x) d y-\sin (n \pi x) d t=0, \quad(x, y, t) \in \mathbf{S}^{1} \times \mathbf{C} \times \mathbf{R},
$$

où $n$ est un entier strictement positif, pair dès que $\xi$ est orientable. Le nombre $-n / 2$ n'est autre que l'enroulement de la structure autour des cercles legendriens qui fibrent $\mathrm{F}$.

Le lemme qui suit est une version relative d'un résultat établi dans [Gi1].

Lemme 2.7. Soit $\mathrm{F} \subset(\mathrm{M}, \xi)$ une surface convexe, $U$ un voisinage tubulaire de $\mathrm{F}$ homogène, $\mathrm{P}$ un compact de $\mathrm{M}$ dont l'intersection avec $\mathrm{F}$ est saturée par $\xi \mathrm{F}$ et $\sigma$ un feuilletage de $\mathrm{F}$ scindé par $\Gamma_{U}$ et égal à $\xi \mathrm{F}$ près de $\mathrm{P} \cap \mathrm{F}$. Il existe alors une isotopie de plongements $\phi_{t}: \mathrm{F} \rightarrow U, t \in[0,1]$, ayant les propriétés suivantes:

1) $\phi_{0}$ est l'inclusion;

2) pour tout $t \in[0,1]$, la surface $\phi_{t}(\mathrm{~F})$ est transversale aux fibres de $U$;

3) le feuilletage caractéristique $\xi \phi_{1}(\mathrm{~F})$ n'est autre que $\left(\phi_{1}\right)_{*} \sigma$;

4) l'intersection $\phi_{t}(\mathrm{~F}) \cap \mathrm{P}$ coïncide avec $\mathrm{F} \cap \mathrm{P}$ pour tout $t \in[0,1]$. 
Démonstration. La proposition II.3.6 de [Gi1] donne une isotopie $\bar{\phi}_{t}$ qui satisfait aux conditions 1-3) et laisse fixe un voisinage de $\mathrm{F} \cap \mathrm{P}$. Pour obtenir 4), on remarque qu'on garde 1-3) si on compose $\bar{\phi}_{t}$ au but par une isotopie de contact partant de l'identité et préservant la structure fibrée du tube $U$. On note alors $\left.\left.\delta_{s}: U \simeq \mathrm{F} \times \mathbf{R} \rightarrow U, s \in\right] 0,1\right]$, l'homothétie de rapport $s$ dans les fibres et on observe que, pour $s_{0}$ assez petit, toutes les surfaces $\delta_{s_{0}} \circ \bar{\phi}_{t}(\mathrm{~F}), t \in[0,1]$, coupent $\mathrm{P}$ exactement suivant $\mathrm{F} \cap \mathrm{P}$. D'autre part, comme $\xi$ est $\mathbf{R}$-invariante dans $U$, elle $\mathrm{y}$ admet une équation de Pfaff du type $\beta+u d t=0$, où $t$ décrit $\mathbf{R}$ et $\beta, u$ sont respectivement une 1 -forme et une fonction sur $\mathrm{F}$. Chaque structure $\xi_{s}=\left(\delta_{s}\right)_{*} \xi$ a ainsi pour équation $\beta+(u / s) d t=0$ et est donc encore $\mathbf{R}$-invariante. La méthode du chemin fournit alors une isotopie $\psi_{s}$ de $U, s \in\left[s_{0}, 1\right]$, qui est $\mathbf{R}$-équivariante, envoie $\xi_{s}$ sur $\xi=\xi_{1}$ et déplace les points horizontalement en respectant tous les feuilletages $\xi_{s}(\mathrm{~F} \times\{*\})=\xi(\mathrm{F} \times\{*\})$. En particulier, comme l'intersection $\mathrm{F} \cap \mathrm{P}$ est saturée par $\xi \mathrm{F}$, elle est préservée par l'isotopie $\psi_{s}$. On prend alors une fonction lisse $s:[0,1] \rightarrow] 0,1]$ qui vaut 1 en 0 mais devient vite très petite. L'isotopie

$$
\phi_{t}=\psi_{s(t)} \circ \delta_{s(t)} \circ \bar{\phi}_{t}: \mathrm{F} \longrightarrow U
$$

vérifie toutes les propriétés voulues.

\section{E. Redressement des tores}

Un ingrédient clé dans la thèse de W. Thurston [Th2] est le résultat suivant, dû indépendamment à $\mathrm{R}$. Roussarie [Ro] : dans une variété de dimension 3 munie d'un feuilletage de codimension 1 sans composantes de Reeb, tout tore incompressible plongé est isotope à une feuille ou à un tore transversal au feuilletage. Dans les variétés de contact, les techniques de [Gi1] permettent d'établir un fait analogue très utile pour démontrer le théorème 2.3 :

Lemme 2.8. Soit $\xi$ une structure de contact sur $\mathrm{V}$ et $\mathrm{R} \subset \mathrm{S}$ une sous-surface compacte, connexe et à bord non vide. Si l'enroulement $\mathrm{e}(\xi)$ de $\xi$ est strictement négatif, $\xi$ est isotope à une structure de contact $\xi^{\prime}$ pour laquelle, au-dessus de $\mathrm{R}$, toutes les fibres sont legendriennes et ont un enroulement égal $\grave{a} \mathrm{e}(\xi)$.

Démonstration. On regarde la surface $\mathrm{R}$ comme un voisinage régulier d'un bouquet de cercles $\mathrm{K}=\bigvee_{i=1}^{k} \mathrm{~K}_{i}$ dans $\mathrm{S}$ et on note $q$ le sommet de $\mathrm{K}$. Quitte à faire une première isotopie, on suppose que la fibre $\mathrm{L}$ au-dessus de $q$ est legendrienne et que son enroulement vaut $\mathrm{e}(\xi)$. Pour tout entier $n>0$, on peut trouver, sur un voisinage tubulaire $\mathrm{W}$ de $\mathrm{L}$, des coordonnées $(x, y, t) \in \mathbf{D}^{2} \times \mathbf{S}^{1}$ dans lesquelles $\mathrm{L}=\{0\} \times \mathbf{S}^{1}$ et $\xi$ a pour équation

$$
\cos (2 n \pi t) d x-\sin (2 n \pi t) d y=0 .
$$

La projection $\pi_{\mathrm{W}}: \mathrm{W} \rightarrow \mathbf{D}^{2}, \quad(x, y, t) \mapsto(x, y)$, est alors une fibration legendrienne et l'enroulement de $\xi$ autour des fibres de $\pi_{\mathrm{W}}$ vaut $-n$. Ainsi, pour 
$n=-\mathrm{e}(\xi)$ (qui est un entier strictement positif), $\pi_{\mathrm{W}}$ induit la même trivialisation normale de $\mathrm{L}$ que la fibration $\pi: \mathrm{V} \rightarrow \mathrm{S}$. Il existe donc une isotopie $\phi_{t}: \mathrm{V} \rightarrow \mathrm{V}, t \in[0,1]$, qui a les propriétés suivantes :

- $\phi_{0}=\mathrm{id}$;

- $\phi_{t}(\mathrm{~L})=\mathrm{L}$ pour tout $t \in[0,1]$;

- $\phi_{1}$ envoie chaque fibre de $\pi_{\mathrm{W}}$ sur une fibre de $\pi$.

Quitte à remplacer $\xi$ par $\left(\phi_{1}\right)_{*} \xi$, on suppose désormais que $\xi$ est tangente aux fibres de $\mathrm{V}$ au-dessus d'un voisinage compact $Q$ de $q$. On réduit $Q$ au besoin pour que $Q \cap \mathrm{K}$ soit connexe. Une version relative facile des résultats de [Gi1] permet alors de déformer $\xi$ par une $\mathcal{C}^{\infty}$-petite isotopie relative à $\mathrm{M}=\pi^{-1}(Q)$ pour que chaque anneau $\pi^{-1}\left(\mathrm{~K}_{i} \backslash Q\right), 1 \leq i \leq k$, soit convexe. On lisse ensuite chaque $\mathrm{K}_{i}$ dans $Q$ en une courbe $\mathrm{K}_{i}^{\prime}$. Les tores $\mathrm{F}_{i}=\pi^{-1}\left(\mathrm{~K}_{i}^{\prime}\right)$ sont alors convexes. On en prend des voisinages homogènes respectifs $U_{i}$ et on pose $\Gamma_{i}=\Gamma_{U_{i}}$ (cf. définition 2.6).

Assertion. L'intersection géométrique $\operatorname{Card}\left(\mathrm{L} \cap \Gamma_{i}\right)$ de $\mathrm{L}$ avec $\Gamma_{i}$ est égale au module $\left|[\mathrm{L}] \cdot\left[\Gamma_{i}\right]\right|$ de leur intersection algébrique - toutes les composantes connexes de $\Gamma_{i}$ étant orientées dans le même sens.

Preuve. L'enroulement $\mathrm{e}(\mathrm{L})$ se lit sur $\mathrm{F}_{i}$ comme - $(1 / 2) \operatorname{Card}\left(\mathrm{L} \cap \Gamma_{i}\right)$. Si l'assertion est fausse, $\mathrm{F}_{i}$ porte une courbe fermée simple $\mathrm{C}$ isotope à $\mathrm{L}$ qui intersecte $\Gamma_{i}$ moins que $\mathrm{L}$ (géométriquement). On peut alors construire sans peine sur $\mathrm{F}_{i}$ un feuilletage singulier $\sigma$ qui est scindé par $\Gamma_{i}$ et pour lequel la courbe $\mathrm{C}$ est saturée (voir [Gi1, exemple II.3.7]). Le lemme II.3.6 de [Gi1] (version absolue du lemme 2.7) fournit alors un plongement $\phi$ de $\mathrm{F}_{i}$ dans $U_{i}$ - isotope à l'inclusion dont l'image a pour feuilletage caractéristique $\phi_{*} \sigma$. L'enroulement de $\xi$ autour de la courbe legendrienne $\mathrm{L}^{\prime}=\phi(\mathrm{C})$ vaut alors

$$
\mathrm{e}\left(\mathrm{L}^{\prime}\right)=-\frac{1}{2} \operatorname{Card}\left(\mathrm{C} \cap \Gamma_{i}\right)>-\frac{1}{2} \operatorname{Card}\left(\mathrm{L} \cap \Gamma_{i}\right)=\mathrm{e}(\mathrm{L}),
$$

ce qui contredit le fait que $\mathrm{e}(\mathrm{L})$ est égal à $\mathrm{e}(\xi)$.

L'assertion ci-dessus permet de déformer $\xi$, par une isotopie relative à $M$ laissant les tores $\mathrm{F}_{i}$ invariants, de telle sorte que chaque fibre de $\left.\pi\right|_{\mathrm{F}_{i}}$ ait, avec $\Gamma_{i}$, une intersection géométrique égale au module de son intersection algébrique. Cette condition étant remplie, il existe sur $\mathrm{F}_{i}$ un feuilletage singulier $\sigma_{i}$ ayant les propriétés suivantes :

- $\sigma_{i}$ est scindé par $\Gamma_{i}$;

- $\sigma_{i}$ coïncide avec $\xi \mathrm{F}_{i}$ dans $\mathrm{F}_{i} \cap \mathrm{M}$;

- chaque fibre de $\left.\pi\right|_{F_{i}}$ est saturée par $\sigma_{i}$.

Le lemme 2.7 donne alors, pour $1 \leq i \leq k$, un plongement $\phi_{i}$ de $\mathrm{F}_{i}$ dans $U_{i}$ - isotope à l'inclusion - dont l'image a pour feuilletage caractéristique $\left(\phi_{i}\right)_{*} \sigma_{i}$ et a même intersection que $\mathrm{F}_{i}$ avec le compact

$$
\mathrm{P}_{i}=\mathrm{M} \cup \phi_{1}\left(\mathrm{~F}_{1}\right) \cup \cdots \cup \phi_{i-1}\left(\mathrm{~F}_{i-1}\right) \cup \mathrm{F}_{i+1} \cup \cdots \cup \mathrm{F}_{k} .
$$


Il existe donc un difféomorphisme $\phi$ de $\mathrm{V}$, isotope à l'identité, qui « prolonge » simultanément tous les plongements $\phi_{i}$. Par construction, la structure de contact $\phi^{*} \xi$ imprime le feuilletage $\sigma_{i}$ sur chaque tore $\mathrm{F}_{i}$ et est ainsi tangente aux fibres de $\pi$ au-dessus de $Q \cup \mathrm{K}$. Comme tous les tores $\mathrm{F}_{i}$ sont convexes (voire clairaldiens), il est facile de rendre la fibration $\pi$ legendrienne au-dessus de $\mathrm{R}$ par une ultime isotopie relative à $\pi^{-1}(Q \cup \mathrm{K})$.

\section{F. Structures de contact sur le tore plein}

La démonstration de la proposition 2.5 passe par une analyse des structures de contact tendues sur le tore plein $\mathrm{W}=\mathbf{D}^{2} \times \mathbf{S}^{1}$. Cette analyse est menée dans [Gi4] et on en présente ici quelques conclusions utiles. Pour cela, on rappelle qu'un feuilletage du tore $\mathbf{T}^{2}$ est une suspension s'il est non singulier et si toutes ses feuilles coupent une même courbe transversale fermée, simple et connexe. D'autre part, on observe qu'une structure de contact sur $\mathbf{D}^{2} \times \mathbf{S}^{1}$ est orientable si et seulement si elle l'est le long des fibres de la projection $\mathbf{D}^{2} \times \mathbf{S}^{1} \rightarrow \mathbf{D}^{2}$.

Lemme 2.9. Soit $\xi$ une structure de contact orientable et tendue sur $\mathrm{W}=\mathbf{D}^{2} \times$ $\mathbf{S}^{1}$. On suppose que le feuilletage caractéristique $\xi \partial \mathrm{W}$ est scindé par une multicourbe ayant $2 n$ composantes connexes et que ses éventuelles singularités forment des cercles lisses. Il existe alors $n$ anneaux disjoints $\mathrm{A}_{i}$ plongés dans $\mathrm{W}$ et ayant les propriétés suivantes:

- chaque composante de $\partial \mathrm{A}_{i}$ est une courbe de singularités ou une feuille fermée de $\xi \partial \mathrm{W}$;

- $\quad$ chaque feuilletage $\xi \mathrm{A}_{i}$ est constitué de cercles parallèles au bord.

Démonstration. Si le feuilletage $\xi \partial \mathrm{W}$ est une suspension, les anneaux $\mathrm{A}_{i}$ sont directement fournis par la proposition 3.15 de [Gi4] : dans la terminologie de cet article, ce sont les anneaux du feuillage d'une structure de contact élémentaire isotope à $\xi$ relativement au bord. On va maintenant adapter l'argument au cas où $\xi \partial \mathrm{W}$ est un feuilletage scindé dont les singularités forment des cercles. Dans ce cas, l'étude des surfaces convexes (voir les sections 2.B et 2.C de [Gi4]) montre que $\xi$ est isotope, relativement au bord, à une structure de contact $\xi^{\prime}$ ayant les propriétés suivantes :

- chaque tore $\mathrm{T}_{a}=a \mathbf{S}^{1} \times \mathbf{S}^{1}, 1 / 2 \leq a \leq 1$, est convexe dans $\left(\mathrm{W}, \xi^{\prime}\right)$;

- le feuilletage $\xi \mathrm{T}_{1 / 2}$ est une suspension;

- les singularités éventuelles de chaque feuilletage $\xi^{\prime} \mathrm{T}_{a}, 1 / 2<a \leq 1$, forment des cercles.

Dans $\mathrm{W}^{\prime}=(1 / 2) \mathbf{D}^{2} \times \mathbf{S}^{1}$, la proposition 3.15 de [Gi4] donne, comme avant, des anneaux $\mathrm{A}_{i}^{\prime}$ qui conviennent pour la restriction de $\xi^{\prime}$. D'autre part, dans $\mathrm{W} \backslash$ Int $\mathrm{W}^{\prime}$, l'union des feuilles fermées et des singularités de tous les feuilletages $\xi^{\prime} \mathrm{T}_{a}, 1 / 2 \leq a \leq 1$, forme $2 n$ anneaux disjoints qui complètent les $\mathrm{A}_{i}^{\prime}$ en les anneaux $\mathrm{A}_{i}$ cherchés. 
Proposition 2.10. Soit $\xi$ une structure de contact orientable et universellement tendue sur $\mathrm{W}=\mathbf{D}^{2} \times \mathbf{S}^{1}$. On suppose que le feuilletage caractéristique $\xi \partial \mathrm{W}$ est scindé par deux courbes et que ses singularités forment deux cercles lisses. La structure $\xi$ est alors isotope, relativement au bord, à une structure de contact qui est transversale à $\{0\} \times \mathbf{S}^{1}$ et imprime une suspension sur chaque tore a $\mathbf{S}^{1} \times \mathbf{S}^{1}$, $0<a<1$.

Démonstration. Soit $\sigma$ un feuilletage de $\partial \mathrm{W}$ scindé par deux courbes et dont les singularités forment deux cercles lisses. D'après le théorème 1.6 de [Gi4], complété par le lemme 3.13, les structures de contact universellement tendues sur W qui impriment $\sigma$ sur $\partial \mathrm{W}$ forment au plus deux classes d'isotopie relative au bord. Chaque classe est caractérisée par la classe d'isotopie de l'anneau que fournit le lemme 2.9. Autrement dit, il y a deux classes (resp. une) s'il y a dans W, à isotopie relative au bord près, deux anneaux (resp. un seul) qui s'appuient sur les cercles singuliers de $\sigma$. On exhibe ci-dessous des structures de contact universellement tendues explicites dans chaque classe et on constate qu'elles satisfont les propriétés requises.

Sur $\mathbf{R}^{2} \times \mathbf{S}^{1}$ muni de coordonnées cylindriques $(r, \theta, z), z \in \mathbf{R} / 2 \pi \mathbf{Z}$, l'équation de Pfaff $\left(1-r^{4}\right) d z+r^{2} d \theta=0$ définit une structure de contact $\zeta$ universellement tendue (l'équation de $\zeta$ définit sur $\mathbf{R}^{3}$ la structure de contact ordinaire). De plus, pour tout $r>0$, le feuilletage caractéristique du tore de rayon $r$ autour de $\{0\} \times \mathbf{S}^{1}$ est le feuilletage linéaire de pente $d z / d \theta=r^{2} /\left(r^{4}-1\right)$. Étant donné des entiers $p$ et $q$ premiers entre eux, $q>0$, il existe donc un unique réel $r=r(p, q)$ tel que les caractéristiques du tore $\partial\left(r \mathbf{D}^{2}\right) \times \mathbf{S}^{1}$ aient pour classe d'homologie $(p, q)$. On considère alors les plongements $\psi_{p, q}^{ \pm}: \mathrm{W}^{\prime}=(1 / 2) \mathbf{D}^{2} \times \mathbf{S}^{1} \rightarrow \mathbf{R}^{2} \times \mathbf{S}^{1}$ donnés par

$$
\psi_{p, q}^{ \pm}\left(a e^{i s}, t\right)=\left(2 a r(p, q)\left(1 \pm \frac{a}{q} \cos (q s-p t)\right), s+\frac{a}{q} \sin (2(q s-p t)), t\right) .
$$

Les structures de contact induites, $\zeta_{ \pm}=\left(\psi_{p, q}^{ \pm}\right)^{*} \zeta$, sont universellement tendues, transversales à $\{0\} \times \mathbf{S}^{1}$ et impriment une suspension sur chaque tore $\mathrm{T}_{a}=$ $a \mathbf{S}^{1} \times \mathbf{S}^{1}, 0<a<1 / 2$. En outre, les feuilletages $\sigma_{ \pm}=\zeta_{ \pm} \partial \mathrm{W}^{\prime}$ sont tous deux scindés par deux courbes et ont deux cercles de singularités qui sont communs et qu'on note $\mathrm{C}_{0}, \mathrm{C}_{1}$. On observe d'autre part que l'image inverse du tore de rayon $r(p, q)$ par $\psi_{p, q}^{ \pm}$est un anneau $\mathrm{A}_{ \pm}$qui a les propriétés décrites au lemme 2.9. De plus, $\mathrm{A}_{-}$n'est isotope à $\mathrm{A}_{+}$relativement à son bord que si $q=1$.

On suppose maintenant que $(p, q)$ est la classe des cercles singuliers de $\sigma$ et on choisit dans $\mathrm{W} \backslash$ Int $\mathrm{W}^{\prime}$ deux anneaux $\mathrm{B}_{0}, \mathrm{~B}_{1}$ qui sont transversaux aux tores $\mathrm{T}_{a}=a \mathbf{S}^{1} \times \mathbf{S}^{1}, 1 / 2 \leq a \leq 1$, et qui s'appuient d'un côté sur les cercles singuliers de $\sigma$, de l'autre sur $\mathrm{C}_{0}$ et $\mathrm{C}_{1}$. Il existe alors sur $\mathrm{W} \backslash$ Int $\mathrm{W}^{\prime}$ deux structures de contact $\eta_{-}$et $\eta_{+}$satisfaisant aux conditions suivantes (voir [Gi4, lemme 2.3]) :

- $\eta_{ \pm} \partial \mathrm{W}^{\prime}=\sigma_{ \pm}$et $\eta_{ \pm} \partial \mathrm{W}=\sigma$;

- chaque feuilletage $\eta_{ \pm} \mathrm{T}_{a}, 1 / 2 \leq a \leq 1$, est scindé et a deux cercles de singularités, à savoir les cercles $\mathrm{B}_{i} \cap \mathrm{T}_{a}, i=0,1$. 



Figure 1. Pour $q=5$, les images respectives par $\psi_{p, q}^{+}$et $\psi_{p, q}^{-}$des cercles de rayons $1 / 6,1 / 3$ et $1 / 2$ dans $\mathbf{D}^{2} \times\{0\}$.

Les structures de contact $\xi_{ \pm}=\zeta_{ \pm} \cup_{\partial \mathrm{W}^{\prime}} \eta_{ \pm}$sont universellement tendues et ne sont isotopes, relativement au bord, que si les anneaux $\mathrm{A}_{ \pm} \cup \mathrm{B}_{0} \cup \mathrm{B}_{1}$ le sont (i.e. si $q=1)$. En outre, elles sont transversales à $\{0\} \times \mathbf{S}^{1}$, impriment $\sigma$ sur le bord $\partial \mathrm{W}$ et une suspension sur $\mathrm{T}_{a}$ pour tout $\left.a \in\right] 0,1 / 2[$. Sans détruire ces propriétés, une $\mathcal{C}^{\infty}$-petite isotopie convenable - à support dans un voisinage de $\left(\mathrm{B}_{0} \cup \mathrm{B}_{1}\right) \cap \mathrm{Int} \mathrm{W}$ - permet de perturber $\xi_{ \pm}$en une structure de contact qui imprime une suspension sur tous les tores $\mathrm{T}_{a}, 0<a<1$.

\section{G. Mise en position transversale}

On démontre ici la proposition 2.5 lorsque $\mathrm{S}$ n'est pas une sphère. Dans un premier temps, $\xi$ désigne juste une structure de contact d'enroulement strictement négatif sur $\mathrm{V}$. On note $g \geq 1$ le genre de $\mathrm{S}$ et $\mathrm{K}$ un bouquet de $2 g$ cercles sur $\mathrm{S}$ ayant pour complémentaire un disque. Compte tenu du lemme 2.8, on suppose que, audessus d'un voisinage compact régulier $\mathrm{R}$ de $\mathrm{K}$, les fibres de $\pi$ sont legendriennes et ont pour enroulement $\mathrm{e}(\xi)$. On note $\mathrm{D}$ le disque fermé $\mathrm{S} \backslash \mathrm{Int} \mathrm{R}$ et $\mathrm{W}$ le tore plein $\pi^{-1}(\mathrm{D})$ qu'on paramètre par $\mathbf{D}^{2} \times \mathbf{S}^{1}$ de telle sorte que la fibration $\pi \mid \mathrm{w}$ soit la projection sur le premier facteur. Par construction, $\partial \mathrm{W}$ est un tore clairaldien.

Lemme 2.11. La multi-courbe $\Gamma$ qui scinde le feuilletage $\xi \partial \mathrm{W}$ a deux composantes connexes.

Démonstration. Soit $2 n$ le nombre (pair) de composantes connexes de $\Gamma$. D'après le lemme 2.9 , les $2 n$ courbes de singularités de $\xi \partial \mathrm{W}$ bordent $n$ anneaux $\mathrm{A}_{i}$ disjoints et plongés dans $\mathrm{W}$ dont les feuilletages caractéristiques $\xi \mathrm{A}_{i}$ sont formés de cercles parallèles au bord. On indexe les $A_{i}$ de telle sorte que $A_{1}$ soit extérieurissime, c'est-à-dire découpe $\mathrm{W}$ en deux tores pleins dont l'un, noté $\mathrm{W}_{1}$, se rétracte par déformation sur $\mathrm{A}_{1}$ et ne contient aucun $\mathrm{A}_{i}, i>1$. On prend ensuite un voisinage collier $N_{1} \cong \mathrm{A}_{1} \times[0,1]$ de $\mathrm{A}_{1}=\mathrm{A}_{1} \times\{0\}$ dans $\mathrm{Adh}\left(\mathrm{W} \backslash \mathrm{W}_{1}\right)$ dont le bord latéral $\partial \mathrm{A}_{1} \times[0,1]$ est inclus dans $\partial \mathrm{W}$. Pour tout $s \neq 0$ assez petit, les caractéristiques de l'anneau $\mathrm{A}_{1} \times\{s\}$ vont d'un bord à l'autre. On désigne 
alors par $\mathrm{W}^{\prime}$ un tore plein obtenu en arrondissant les angles de

$$
\mathrm{W} \backslash\left(\mathrm { W } _ { 1 } \cup \left(\mathrm{A}_{1} \times[0, s[)), \quad s>0\right.\right. \text { petit. }
$$

Ainsi construit, $\mathrm{W}^{\prime}$ est un tore plein isotope à $\mathrm{W}$ et on note $\psi: \partial \mathrm{W} \rightarrow \mathrm{V}$ un plongement isotope à l'inclusion et dont l'image est le tore $\partial \mathrm{W}^{\prime}$.

Si $n>1$, le feuilletage $\xi \partial \mathrm{W}^{\prime}$ a $2 n-2$ courbes de singularités et aucune feuille régulière fermée. Par suite, $\partial \mathrm{W}^{\prime}$ est convexe et la multi-courbe $\Gamma^{\prime}$ qui scinde $\xi \partial \mathrm{W}^{\prime}$ compte $2 n-2$ composantes connexes, toutes isotopes aux composantes de $\psi(\Gamma)$. En notant L une fibre de $\pi$ dans $\partial \mathrm{W}$, il existe sur $\partial \mathrm{W}^{\prime}$ une courbe $\mathrm{L}^{\prime}$ isotope à $\psi(\mathrm{L})$ et vérifiant

$$
\operatorname{Card}\left(\mathrm{L}^{\prime} \cap \Gamma^{\prime}\right)<2|\mathrm{e}(\xi)|=\operatorname{Card}(\mathrm{L} \cap \Gamma) .
$$

Or le lemme 2.7 fournit une isotopie de plongements $\phi_{s}: \partial \mathrm{W}^{\prime} \rightarrow \mathrm{V}, s \in[0,1]$, ayant les propriétés suivantes :

- chaque feuilletage $\xi \phi_{s}\left(\partial \mathrm{W}^{\prime}\right), s \in[0,1]$, est scindé par $\phi_{s}\left(\Gamma^{\prime}\right)$;

- la courbe $\phi_{1}\left(\mathrm{~L}^{\prime}\right)$ est legendrienne.

Comme dans la preuve du lemme 2.8, l'enroulement de $\xi$ autour de $\phi_{1}\left(\mathrm{~L}^{\prime}\right)$ vaut alors

$$
\mathrm{e}\left(\phi_{1}\left(\mathrm{~L}^{\prime}\right)\right)=-\frac{1}{2} \operatorname{Card}\left(\mathrm{L}^{\prime} \cap \Gamma^{\prime}\right)>\mathrm{e}(\xi),
$$

ce qui contredit la définition de e $(\xi)$.

Le second ingrédient dans la preuve de la proposition 2.5 est le lemme suivant :

Lemme 2.12. Si la structure de contact $\xi$ est universellement tendue, sa restriction au tore plein $\mathrm{W}=\pi^{-1}(\mathrm{D})$ l'est aussi.

Démonstration. Soit $n \geq 1$ un entier quelconque. Comme la surface $\mathrm{S}$ n'est pas une sphère, elle possède un revêtement connexe à $n$ feuillets $\mathrm{S}_{n}$. Le rappel $\mathrm{V}_{n} \rightarrow$ $\mathrm{S}_{n}$ du fibré $\mathrm{V} \rightarrow \mathrm{S}$ au-dessus de $\mathrm{S}_{n}$ a alors pour nombre d'Euler $\chi\left(\mathrm{V}_{n}, \mathrm{~S}_{n}\right)=$ $n \chi(\mathrm{V}, \mathrm{S})$. Par suite, il existe un revêtement fibré à $n$ feuillets $\tilde{\mathrm{V}}_{n} \rightarrow \mathrm{V}_{n}$ qui, audessus de chaque fibre de la projection $\mathrm{V}_{n} \rightarrow \mathrm{S}_{n}$, induit un revêtement cyclique non trivial du cercle. L'image inverse de $\mathrm{W}$ dans $\tilde{\mathrm{V}}_{n}$ est la réunion disjointe de $n$ tores pleins et chacun d'eux se projette sur $\mathrm{W}$ par un revêtement de degré $n$. Comme $\xi$ se relève sur $\tilde{\mathrm{V}}_{n}$ en une structure de contact tendue, sa restriction à $\mathrm{W}$ induit une structure de contact tendue sur tout revêtement fini de $\mathrm{W}$, donc aussi sur le revêtement universel.

On termine maintenant la démonstration de la proposition 2.5. On note donc $\xi$ une structure de contact sur $\mathrm{V}$ qui est orientable le long des fibres, universellement tendue et d'enroulement strictement négatif. En outre, compte tenu des lemmes $2.8,2.11,2.12$ et de la proposition 2.10 , on suppose que $\xi$ satisfait les propriétés suivantes :

- toutes les fibres au-dessus du voisinage $\mathrm{R}$ de $\mathrm{K}$ sont legendriennes; 
- dans $\mathrm{W}=\pi^{-1}(\mathrm{D}) \cong \mathbf{D}^{2} \times \mathbf{S}^{1}$, la structure de contact $\xi$ est transversale à $\{0\} \times \mathbf{S}^{1}$ et imprime une suspension sur chaque tore $\mathbf{T}_{a}=a \mathbf{S}^{1} \times \mathbf{S}^{1}, 0<a<1$.

Comme l'enroulement $\mathrm{e}(\xi)$ est strictement négatif, les feuilletages $\xi \mathrm{T}_{a}, 0<a<$ 1 , n'ont aucune feuille fermée isotope à la fibre et sont par suite tous isotopes à des feuilletages transversaux aux fibres. Quitte à déformer $\xi$ par une isotopie à support dans $\mathrm{W}$, on peut donc supposer que toutes les fibres, au-dessus de Int D, sont transversales à $\xi$. On oriente alors $\xi$ sur un voisinage de $\mathrm{W}$ pour que les dites fibres $\{*\} \times \mathbf{S}^{1} \subset$ Int $\mathrm{W}$ soient des transversales positives. On se donne par ailleurs un champ de vecteurs legendrien $\tau$ sur $\mathrm{V}$ qui est nul sur $\mathrm{W}$ et transversal aux fibres sur $\mathrm{V} \backslash \mathrm{W}$. Si on pousse $\xi$ par le flot de $\tau$ pendant un bref instant, on obtient une structure de contact $\xi^{\prime}$ qui est partout transversale aux fibres sauf le long de $\partial \mathrm{W}$ où elle reste tangente aux fibres. De plus, si on pousse dans la bonne direction, les fibres de part et d'autre de $\partial \mathrm{W}$ sont transversales dans le même sens. Une isotopie $\mathcal{C}^{\infty}$-petite permet alors de rendre $\xi^{\prime}$ transversale à toutes les fibres.

\section{H. Un exemple}

Pour clore cette partie, on montre que l'alternative offerte par le théorème 2.3 est optimale au sens où, lorsque $\xi$ n'est pas isotope à une structure transversale aux fibres, il est parfois indispensable de passer à un revêtement fini de $\mathrm{V}$ pour trouver une courbe legendrienne isotope à la fibre et d'enroulement nul.

Proposition 2.13. Si S est un tore et si $\chi(\mathrm{V}, \mathrm{S})$ est un nombre négatif assez grand, $\mathrm{V}$ porte une structure de contact virtuellement vrillée d'enroulement strictement négatif.

Démonstration. Soit $\zeta$ la structure de contact d'équation $d z-y d x=0$ sur $\mathbf{R}^{3}$. On se donne un nœud $\mathrm{K}$ transversal à $\zeta$ et un voisinage tubulaire $\mathrm{W}$ de $\mathrm{K}$ dans lequel $\zeta$ a pour équation $d t+r^{2} d \theta=0$, où $t$ paramètre $\mathrm{K}$ et $(r, \theta), r \leq \varepsilon$, sont des coordonnées polaires normales. On note $\mathrm{I}(\mathrm{K})$ l'auto-enlacement de $\mathrm{K}$ dans $\left(\mathbf{R}^{3}, \zeta\right)$, enlacement de $\mathrm{K}$ avec $\mathrm{K}+\varepsilon \partial_{y}$, et $\mathrm{K}^{\prime}$ une stabilisation de $\mathrm{K}$ dans $\mathrm{W}$, c'est-à-dire un nœud topologiquement isotope à $\mathrm{K}$ dans $\mathrm{W}$, transversal à $\zeta$ et d'auto-enlacement $l\left(K^{\prime}\right)=1(K)-2$ (voir $\left.[B e]\right)$. Le théorème de Darboux assure alors qu'il existe un isomorphisme $\phi$ de $(\mathrm{W}, \zeta)$ sur un voisinage tubulaire $\left(\mathrm{W}^{\prime}, \zeta\right)$ de $\mathrm{K}^{\prime}$ qu'on peut prendre aussi petit qu'on veut. Comme $\phi$ envoie les méridiens de $\mathrm{W}$ sur des méridiens de $\mathrm{W}^{\prime}$, la variété $\mathrm{V}$ obtenue à partir de $\mathrm{W} \backslash \mathrm{Int} \mathrm{W}^{\prime}$ en identifiant par $\phi$ les deux composantes du bord est fibrée en cercles au-dessus du tore. On note $\xi$ la structure de contact induite par $\zeta$ sur $\mathrm{V}$ et on observe que les propriétés suivantes sont satisfaites :

- le nombre d'Euler de V est négatif, d'autant plus grand que $\mathrm{W}^{\prime}$ est plus petit ; 
- au signe près, la classe d'Euler de $\xi$ est en dualité de Poincaré avec la fibre de V.

Cette seconde propriété montre immédiatement que $\xi$ n'est pas isotope à une structure transversale aux fibres. Par ailleurs, si $(\mathrm{V}, \xi)$ contenait une courbe legendrienne isotope à la fibre et d'enroulement nul, celle-ci se relèverait - peutêtre pas dans $\mathrm{W} \backslash \mathrm{W}^{\prime}$ mais dans $\mathrm{W} \backslash \phi^{n}\left(\mathrm{~W}^{\prime}\right)$ pour $n$ assez grand - en une courbe legendrienne de $(\mathrm{W}, \zeta)$ bordant un disque méridien vrillé. Or la structure $\zeta$ est tendue d'après le théorème de Bennequin.

$\mathrm{Au}$ prix de quelques efforts supplémentaires, l'exemple ci-dessus révèle aussi qu'on ne peut pas se contenter de considérer des revêtements finis du type $\tilde{\mathrm{V}}=\rho^{*} \mathrm{~V}$ où $\rho$ est un revêtement fini de $\mathrm{S}$ : il faut en général déplier les fibres.

\section{Dénombrement des structures de contact transversales}

\section{A. Comment contacter Matsumoto-Ghys}

V désigne toujours une variété connexe orientée fibrée en cercles au-dessus d'une surface close S. On classifie ici les structures de contact directes et transversales aux fibres sur V . Pour ce qui est des feuilletages, aucune classification topologique générale n'est connue ni même attendue (voir [Gh1]). Toutefois, lorsque $\chi(\mathrm{V}, \mathrm{S})$ vaut $\pm \chi(\mathrm{S})$, les travaux de S. Matsumoto et É. Ghys montrent que les feuilletages $\mathcal{C}^{2}$ transversaux aux fibres sur $\mathrm{V}$ sont tous topologiquement conjugués [Ma] et forment, à conjugaison différentiable près, une variété homéomorphe à l'espace de Teichmüller de S [Gh2]. Pour les structures de contact, le théorème qui suit donne, sans autre restriction sur $\chi(\mathrm{V}, \mathrm{S})$ que l'inégalité du théorème 1.1, une classification complète. On rappelle que, d'après les propositions 2.4 et 2.5, une structure de contact sur $\mathrm{V}$ est isotope à une structure transversale aux fibres si et seulement si elle est universellement tendue et d'enroulement strictement négatif entier.

Théorème 3.1. Soit V une variété connexe et orientée, fibrée en cercles audessus d'une surface close et orientable $\mathrm{S}$. On suppose que $\chi(\mathrm{S}) \leq 0$ et que $\chi(\mathrm{V}, \mathrm{S}) \leq-\chi(\mathrm{S})$.

a) Il existe sur $\mathrm{V}$ des structures de contact transversales aux fibres et d'enroulement $-n$ si et seulement si $n=1$ ou si $n \chi(\mathrm{V}, \mathrm{S})=-\chi(\mathrm{S})$ et $n>0$.

b) Si $\chi(\mathrm{V}, \mathrm{S}) \neq-\chi(\mathrm{S})$, les structures de contact universellement tendues et d'enroulement -1 sur $\mathrm{V}$ forment une seule classe d'isotopie.

c) Si $n \chi(\mathrm{V}, \mathrm{S})=-\chi(\mathrm{S}), n>0$, les structures de contact universellement tendues et d'enroulement $-n$ forment un nombre fini de classes de conjugaison égal au nombre de diviseurs de $n$. De plus, chaque classe de conjugaison contient une infinité de classes d'isotopie.

Avant de démontrer ce théorème dans les sections $\mathrm{C}-\mathrm{F}$, on observe que la partie c) présente peu d'intérêt lorsque $\chi(\mathrm{S})=0$. En effet, comme $n \chi(\mathrm{V}, \mathrm{S})=$ 
- $\chi(\mathrm{S})$, la variété $\mathrm{V}$ est un tore et l'enroulement n'est pas invariant par conjugaison. Dans ce cas, le bon invariant de conjugaison est la torsion [Gi4]. D'autre part, le cas laissé de côté par le théorème est en fait beaucoup plus simple :

Proposition 3.2. Soit V une variété connexe et orientée, fibrée en cercles audessus d'une sphère $\mathrm{S}$. On suppose que $\chi(\mathrm{V}, \mathrm{S})<0$. $\dot{A}$ isotopie près, il y a sur $\mathrm{V}$ une seule structure de contact directe et transversale aux fibres. Son enroulement vaut -2 si $\mathrm{V} \simeq \mathbf{S}^{3}$ et -1 sinon.

Démonstration. Ce résultat est inclus dans le théorème 1.1 de [Gi4] qui classifie les structures de contact tendues sur les espaces lenticulaires. Comme il n'en constitue qu'une toute petite partie, on indique brièvement sa preuve. Soit $\xi_{0}$ et $\xi_{1}$ deux structures de contact sur $\mathrm{V}$ qui sont directes et transversales aux fibres. Quitte à déformer l'une d'elles par une isotopie qui la laisse transversale aux fibres, on peut supposer que $\xi_{0}$ et $\xi_{1}$ coïncident au-dessus de la réunion disjointe $Q$ de deux petits disques dans $\mathrm{S}$. On paramètre alors $\mathrm{V} \backslash \pi^{-1}(Q)$ par $\mathbf{T}^{2} \times[0,1]$ de telle sorte que $\pi$ soit la projection $\mathbf{T}^{2} \times[0,1] \rightarrow \mathbf{S}^{1} \times[0,1]$, $(x, y, t) \mapsto(y, t)$. Chaque structure $\xi_{i}$ imprime ainsi sur chaque tore $\mathbf{T}^{2} \times\{t\}$ un feuilletage caractéristique dont toutes les feuilles coupent transversalement les fibres de $\pi$. Dans la terminologie de [Gi4], $\xi_{0}$ et $\xi_{1}$ sont des structures rotatives sur $\mathbf{T}^{2} \times[0,1]$ qui coïncident près du bord et ont la même amplitude (non nulle). Le théorème 3.3 de [Gi4] montre qu'elles sont alors isotopes relativement au bord.

\section{B. Fibrations legendriennes et revêtements fibrés}

On étudie ici les structures de contact sur $\mathrm{V}$ qui sont tangentes aux fibres, i.e. pour lesquelles la projection $\pi: \mathrm{V} \rightarrow \mathrm{S}$ est une fibration legendrienne. Comme la proposition 1.4 le laisse entrevoir, ces structures jouent un rôle important dans l'étude des structures de contact transversales aux fibres. On note cependant qu'une structure de contact tangente aux fibres n'est pas nécessairement orientable le long des fibres. L'exemple type est la structure de contact canonique $\xi_{\mathrm{S}}$ sur le fibré $\mathbf{P}(\mathrm{TS})$ des droites non orientées tangentes à $\mathrm{S}$.

Proposition 3.3. Soit V une variété connexe et orientée, fibrée en cercles audessus d'une surface quelconque $\mathrm{S}$. L'application qui, à tout revêtement fibré $\rho: \mathrm{V} \rightarrow \mathbf{P}(\mathrm{TS})$, associe la structure de contact $\xi=\rho^{*} \xi_{\mathrm{S}}$ est une bijection de l'espace des revêtements fibrés et orientés $\mathrm{V} \rightarrow \mathbf{P}(\mathrm{TS})$ dans l'espace des structures de contact tangentes aux fibres sur $\mathrm{V}$. En outre, l'enroulement de $\rho^{*} \xi_{\mathrm{S}}$ autour des fibres vaut $-d / 2$ où $d$ est le degré du revêtement $\rho$.

Démonstration. Si $\rho: \mathrm{V} \rightarrow \mathbf{P}(\mathrm{TS})$ est un revêtement fibré orienté, la structure de contact $\xi=\rho^{*} \xi_{\mathrm{S}}$ est tangente aux fibres de $\mathrm{V}$ (et directe). En outre, comme l'enroulement de $\xi_{\mathrm{S}}$ autour des fibres vaut $-1 / 2$, celui de $\xi$ vaut $-d / 2$ où $d$ 
est le degré de $\rho$. D'autre part, tout champ de plans $\xi$ tangent aux fibres de $\mathrm{V}$ définit une application fibrée $\mathrm{V} \rightarrow \mathbf{P}(\mathrm{TS})$ : l'image d'un point $p$ est simplement la projection sur $\mathrm{S}$ du plan $\xi(p)$. Cette application est un revêtement (orienté) si et seulement si le champ $\xi$ est une structure de contact (directe) et cette structure est, par construction, le rappel de $\xi_{\mathrm{S}}$.

Corollaire 3.4. Soit V une variété connexe et orientée fibrée en cercles audessus d'une surface close $\mathrm{S}$. Pour que $\mathrm{V}$ porte une structure de contact tangente aux fibres (et directe), il faut et il suffit qu'il existe un entier $d>0$ tel que $d \chi(\mathrm{V}, \mathrm{S})=-2 \chi(\mathrm{S})$.

Démonstration. Le nombre d'Euler du fibré $\mathbf{P}(\mathrm{TS}) \rightarrow \mathrm{S}$, pour l'orientation induite par la structure de contact $\xi_{\mathrm{S}}$, est $-2 \chi(\mathrm{S})$. Ainsi, la relation $d \chi(\mathrm{V}, \mathrm{S})=$ $-2 \chi(\mathrm{S})$ traduit simplement l'existence d'un revêtement fibré et orienté de $\mathrm{V}$ sur $\mathbf{P}(\mathrm{TS})$ à $d$ feuillets.

Remarque. Lorsque la surface S est close, l'existence sur V d'un simple champ de plans tangent aux fibres exige en fait déjà que le rapport $-2 \chi(\mathrm{S}) / \chi(\mathrm{V}, \mathrm{S})$ soit un entier, éventuellement négatif ou nul. En effet, ce champ de plans définit une application fibrée $\mathrm{V} \rightarrow \mathbf{P}(\mathrm{TS})$ dont la restriction à chaque fibre est de degré constant $d$. D'autre part, un feuilletage tangent aux fibres ne peut être que l'image inverse d'un feuilletage de $\mathrm{S}$ et n'existe donc que si $\chi(\mathrm{S})=0$.

Corollaire 3.5. Soit V une variété connexe et orientée, fibrée en cercles audessus d'une surface compacte $\mathrm{S}$ ayant un bord connexe non vide. On note $\hat{\mathrm{S}} \subset \mathrm{V}$ l'image d'une section, $\mathrm{L} \subset \partial \mathrm{V}$ une fibre et on oriente $\partial \hat{\mathrm{S}}$ et $\mathrm{L}$ de telle sorte que leur intersection soit positive sur $\partial \mathrm{V}$.

Pour toute structure de contact $\xi$ tangente aux fibres de $\mathrm{V}$, la multi-courbe des singularités de $\xi \partial \mathrm{V}$ a pour classe d'homologie

$$
\pm 2(\mathrm{e}(\xi)[\partial \hat{\mathrm{S}}]+\chi(\mathrm{S})[\mathrm{L}]) \in H_{1}(\partial \mathrm{V} ; \mathbf{Z})
$$

du moins si toutes ses composantes sont orientées dans le même sens.

Démonstration. Si $\mathrm{V}=\mathbf{P}(\mathrm{TS})$ et si $\xi$ est la structure de contact canonique, la courbe des singularités de $\xi \partial \mathrm{V}$ n'est autre que le relèvement legendrien de $\partial \mathrm{S}$ dans $\mathrm{V}$. Comme $\mathrm{e}(\xi)=-1 / 2$, la formule dit simplement que, par rapport à n'importe quel champ de droites défini sur $\mathrm{S}$, la tangente au bord $\partial \mathrm{S}$ fait $-2 \chi(\mathrm{S})$ tours sur la fibre. ${ }^{3}$ Le cas général s'obtient en passant à un revêtement fibré de degré $-2 \mathrm{e}(\xi)$.

Pour terminer cette section, on vérifie que l'enroulement d'une structure de contact tangente aux fibres est bien ce qu'on attend :

3 Pour trouver le bon signe, noter que l'orientation de contact sur $\mathbf{P}(\mathrm{TS})$ en un point $(q, \delta)$, $\delta \subset \mathrm{T}_{q} \mathrm{~S}$, n'est pas la juxtaposition d'une orientation de $\mathrm{T}_{q} \mathrm{~S}$ et de l'orientation induite sur la droite $\mathbf{P}\left(\mathrm{T}_{q} \mathrm{~S}\right)$, mais l'inverse. 
Lemme 3.6. Soit V une variété connexe et orientée, fibrée en cercles au-dessus d'une surface quelconque $\mathrm{S}$, et soit $\xi$ une structure de contact tangente aux fibres. L'enroulement de $\xi$ est égal à son enroulement autour des fibres.

Démonstration. Quitte à se placer au-dessus du revêtement universel de $\mathrm{S}$, on suppose que $S$ est un plan ou une sphère. $S i S \simeq \mathbf{S}^{2}$, il suffit de traiter le cas où $\mathrm{V} \simeq \mathbf{S}^{3}$. Dans ce cas, l'enroulement de $\xi$ autour des fibres est égal à -2 . Par ailleurs, comme on l'a noté au début de la section 2.B, l'enroulement de $\xi$ autour de toute courbe legendrienne $\mathrm{L}$ isotope à la fibre dans $\mathbf{S}^{3}$ - donc non nouée - vaut $\operatorname{tb}(\mathrm{L})-1$. L'identité voulue résulte donc de l'inégalité de Bennequin $\mathrm{tb}(\mathrm{L}) \leq-1$.

Si $\mathrm{S} \simeq \mathbf{R}^{2}$, il existe un difféomorphisme $\mathrm{V} \rightarrow \mathbf{R}^{2} \times \mathbf{S}^{1}$ qui conjugue la fibration $\pi$ à la projection sur $\mathbf{R}^{2}$ et envoie $\xi$ sur la structure de contact d'équation

$$
\cos (n \theta) d x-\sin (n \theta) d y=0, \quad(x, y, \theta) \in \mathbf{R}^{2} \times \mathbf{S}^{1},
$$

où $n$ est l'enroulement de $\xi$ autour des fibres. Dans $\mathbf{R}^{3}$ muni de sa structure de contact ordinaire, on considère alors une courbe legendrienne non nouée L dont l'invariant de Thurston-Bennequin vaut -1 . Un avatar du théorème de Darboux donne un plongement de $(\mathrm{V}, \xi)$ dans $\mathbf{R}^{3}$ qui envoie sur $\mathrm{L}$ une fibre de $\pi$. Toute courbe legendrienne dans $(\mathrm{V}, \xi)$ isotope à la fibre et d'enroulement $-m>-n$ a alors pour image une courbe legendrienne non nouée dont l'invariant de ThurstonBennequin vaut $n-m-1$. L'inégalité de Bennequin permet à nouveau de conclure.

\section{Mise en position tangentielle}

On démontre ici la partie a) du théorème 3.1. On observe d'abord que la structure de contact canonique sur la variété $\mathbf{S}(\mathrm{TS})$ des droites orientées tangentes à $\mathrm{S}$ a pour enroulement -1 (lemme 3.6). Par suite, comme $\chi(\mathrm{V}, \mathrm{S}) \leq-\chi(\mathrm{S})=$ $\chi(\mathbf{S}(\mathrm{TS}), \mathrm{S})$, la chirurgie décrite dans le lemme 1.3 permet de produire sur $\mathrm{V}$ une structure de contact transversale aux fibres et d'enroulement -1 . D'autre part, lorsque $n \chi(\mathrm{V}, \mathrm{S})=-\chi(\mathrm{S})$ pour un certain entier $n$, la variété $\mathrm{V}$ admet un revêtement fibré à $n$ feuillets au-dessus de $\mathbf{S}(\mathrm{TS})$. Ainsi, $\mathrm{V}$ porte une structure de contact tangente aux fibres, d'enroulement $-n$ en vertu du lemme 3.6, que la proposition 1.4 permet de rendre transversale aux fibres. Il reste donc à démontrer la proposition suivante :

Proposition 3.7. Soit V une variété connexe et orientée, fibrée en cercles audessus d'une surface close orientable $\mathrm{S}$, et soit $\xi$ une structure de contact transversale aux fibres et d'enroulement $\mathrm{e}(\xi)=-n, n \in \mathbf{N}$. Si $n \chi(\mathrm{V}, \mathrm{S})=-\chi(\mathrm{S})$, alors $\xi$ est isotope à une structure de contact tangente aux fibres. Sinon, $n$ vaut 1 .

Démonstration. On note $\mathrm{D} \subset \mathrm{S}$ un disque fermé, $\mathrm{R}$ la surface $\mathrm{S} \backslash \operatorname{Int} \mathrm{D}$ et $\mathrm{W}$ le tore plein $\pi^{-1}(\mathrm{D})$. On paramètre $\mathrm{W}$ par $\mathbf{D}^{2} \times \mathbf{S}^{1}$ de telle sorte que la fibration 
$\left.\pi\right|_{\text {W }}$ soit la projection sur $\mathbf{D}^{2}$. En vertu de la proposition 2.4 et du lemme 2.8, $\xi$ est isotope à une structure de contact $\xi^{\prime}$ ayant les propriétés suivantes :

- au-dessus de $\mathrm{R}$, les fibres sont tangentes à $\xi^{\prime}$ et ont pour enroulement $\mathrm{e}\left(\xi^{\prime}\right)=\mathrm{e}(\xi)$;

- au-dessus de Int D, les fibres sont transversales à $\xi^{\prime}$.

D'après le lemme 2.11, les singularités du feuilletage $\xi^{\prime} \partial \mathrm{W}$ forment deux cercles. De plus, vu la définition du nombre d'Euler $\chi(\mathrm{V}, \mathrm{S})$, le corollaire 3.5 montre que la pente de ces cercles sur $\partial \mathrm{W}=\partial \mathbf{D}^{2} \times \mathbf{S}^{1}-$ ou de leurs classes d'homologie dans $H_{1}(\partial \mathrm{W} ; \mathbf{R})=\mathbf{R}^{2}-$ vaut

$$
\mu=\frac{1}{n}(n \chi(\mathrm{V}, \mathrm{S})+\chi(\mathrm{S})-1) .
$$

Assertion. Si $n>1$, alors $n \chi(\mathrm{V}, \mathrm{S})=-\chi(\mathrm{S})$.

Preuve. Soit $\mathrm{T}_{a}, 0<a<1$, le tore $a \mathbf{S}^{1} \times \mathbf{S}^{1} \subset \mathrm{W}=\mathbf{D}^{2} \times \mathbf{S}^{1}$. Le feuilletage caractéristique $\xi^{\prime} \mathrm{T}_{a}$ de chaque tore $\mathrm{T}_{a}$ est transversal aux fibres et est donc décrit par l'application $\phi_{a}$ de premier retour sur une fibre. Quand $a$ varie de 0 à 1 , le nombre de translation $\mu_{a}$ de $\phi_{a}$ décroît continûment de 0 à $\mu$ (proposition 1.2) et n'est nul que pour $a=0$. En particulier, $\mu$ est strictement négatif. Par suite, comme $n=-\mathrm{e}(\xi)>0$ (proposition 2.4), l'entier $n \chi(\mathrm{V}, \mathrm{S})+\chi(\mathrm{S})$ est négatif ou nul.

Si $n>1$ et $n \chi(\mathrm{V}, \mathrm{S})+\chi(\mathrm{S})<0$, la pente $\mu$ est strictement majorée par $-1 /(n-1)$. D'autre part, une petite perturbation de $\xi^{\prime}$ dans $\mathrm{W}$ permet d'imposer à la famille $\phi_{a}$ n'importe quelle propriété générique. On peut ainsi supposer que, pour une valeur $a$, le difféomorphisme itéré $\phi_{a}^{n-1}$ a pour nombre de translation $(n-1) \mu_{a}=-1$ et ne possède que deux points fixes, lesquels sont hyperboliques. Le tore $\mathrm{T}_{a}$ correspondant est alors convexe dans $\left(\mathrm{V}, \xi^{\prime}\right)$ et on note $\Gamma \subset \mathrm{T}_{a}$ une bi-courbe transversale aux fibres qui scinde le feuilletage $\xi^{\prime} \mathrm{T}_{a}$. On choisit ensuite sur $\mathrm{T}_{a}$ un feuilletage singulier $\sigma$ scindé par $\Gamma$ et pour lequel chaque fibre $\mathrm{L}$ de $\left.\pi\right|_{\mathrm{T}_{a}}$ est saturée (voir la démonstration du lemme 2.8). Le lemme 2.7 fournit un plongement $\phi: \mathrm{T}_{a} \rightarrow \mathrm{W}$ isotope à l'inclusion et dont l'image $\mathrm{T}=\phi\left(\mathrm{T}_{a}\right)$ a pour feuilletage caractéristique $\xi^{\prime} \mathrm{T}=\phi_{*} \sigma$. Ainsi, chaque courbe $\phi(\mathrm{L})$ est isotope à la fibre et legendrienne. De plus, comme $\Gamma$ a deux composantes, l'enroulement de $\phi(\mathrm{L})$ vaut $1-n>-n=\mathrm{e}(\xi)$, ce qui est absurde.

On suppose désormais que $n \chi(\mathrm{V}, \mathrm{S})=-\chi(\mathrm{S})$ de sorte que $\mu=-1 / n$. Pour finir la démonstration de la proposition 3.7, il reste à montrer que $\xi$ - ou $\xi^{\prime}$ - est isotope à une structure de contact partout tangente aux fibres. Pour cela, on note d'abord que, d'après le lemme 2.12, la restriction de $\xi^{\prime}$ à W est universellement tendue. Dès lors, le théorème 1.6 de [Gi4] assure que toute structure de contact universellement tendue sur $\mathrm{W}$ qui imprime le même feuilletage que $\xi^{\prime}$ sur $\partial \mathrm{W}$ est isotope à $\xi^{\prime}$ relativement au bord. En effet, la condition $\mu=-1 / n$ garantit qu'il existe dans $\mathrm{W}$, à isotopie relative au bord près, un seul anneau s'appuyant sur les cercles de singularités de $\xi^{\prime} \partial \mathrm{W}$ (voir le début de la démonstration de la 
proposition 2.10). Or la structure d'équation

$$
\cos (n \theta) d x-\sin (n \theta) d y=0, \quad(x, y, \theta) \in \mathbf{D}^{2} \times \mathbf{S}^{1},
$$

définit sur $\mathrm{W}=\mathbf{D}^{2} \times \mathbf{S}^{1}$ une structure de contact universellement tendue, tangente à toutes les fibres et d'enroulement $-n$ autour de chacune.

\section{Structures d'enroulement lâche}

On démontre ici la partie $\mathrm{b})$ du théorème 3.1. On suppose donc que $\chi(\mathrm{V}, \mathrm{S}) \neq$ $-\chi(\mathrm{S})$ et on considère sur $\mathrm{V}$ deux structures de contact $\xi_{0}$ et $\xi_{1}$ universellement tendues et d'enroulement -1 . On note $\mathrm{D}$ un disque fermé dans $\mathrm{S}$ et on regarde la surface $\mathrm{R}=\mathrm{S} \backslash$ Int $\mathrm{D}$ comme le voisinage régulier d'un bouquet de cercles $\mathrm{K}=\mathrm{V}_{i=1}^{2 g} \mathrm{~K}_{i}$ où $g$ est le genre de $\mathrm{S}$. On paramètre en outre le tore plein $\mathrm{W}=\pi^{-1}(\mathrm{D})$ par $\mathbf{D}^{2} \times \mathbf{S}^{1}$ de telle sorte que la fibration $\left.\pi\right|_{\mathrm{W}}$ soit la projection sur $\mathbf{D}^{2}$.

Compte tenu des lemmes $2.8,2.12$ et de la proposition 2.10, on suppose que chaque structure de contact $\xi_{i}, i \in\{0,1\}$, satisfait aux conditions suivantes :

- au-dessus de $\mathrm{R}$, les fibres sont tangentes à $\xi_{i}$ et d'enroulement -1 ;

- au-dessus de Int D, les fibres sont transversales à $\xi_{i}$.

Les singularités du feuilletage $\xi_{i} \partial \mathrm{W}$ forment alors deux cercles dont la classe d'homologie vaut $\pm(1, \chi(\mathrm{V}, \mathrm{S})+\chi(\mathrm{S})-1)$, où $\chi(\mathrm{V}, \mathrm{S})+\chi(\mathrm{S})-1 \leq-2$.

Soit $Q \subset \mathrm{R}$ un voisinage compact du sommet de $\mathrm{K}$ dont l'intersection avec le bouquet $\mathrm{K}$ est connexe. On lisse chaque lacet $\mathrm{K}_{i}$ dans $Q$ en une courbe $\mathrm{K}_{i}^{\prime}$ et on paramètre chaque tore $\mathrm{F}_{i}=\pi^{-1}\left(\mathrm{~K}_{i}^{\prime}\right)$ par $\mathbf{T}^{2}$ de telle sorte que les fibres soient les cercles $\{*\} \times \mathbf{S}^{1}$ et que les (deux) courbes de singularités du feuilletage $\xi_{0} \mathrm{~F}_{i}$ aient pour classe d'homologie $\pm(1,0)$. Les courbes de singularités du feuilletage $\xi_{1} \mathrm{~F}_{i}$ ont alors une classe du type $\pm\left(1, n_{i}\right), n_{i} \in \mathbf{Z}$, et, quitte à composer le paramétrage de $\mathrm{F}_{i}$ par la transformation $\left(x_{1}, x_{2}\right) \in \mathbf{T}^{2} \mapsto\left(-x_{1}, x_{2}\right)$, on prend $n_{i} \geq 0$.

Lemme 3.8. Il existe une structure de contact isotope à $\xi_{1}$ qui coïncide avec $\xi_{0}$ au-dessus de $\mathrm{R}$.

Démonstration. Si les entiers $n_{i}$ sont tous nuls, une isotopie fibrée amène $\xi_{1}$ à coïncider avec $\xi_{0}$ au-dessus de $\mathrm{R}$. On suppose ci-dessous $n_{i} \neq 0$ et on déforme $\xi_{1}$, par une isotopie relative à $\bigvee_{j \neq i} \mathrm{~F}_{j}$, en une structure de contact $\xi_{2}$ dans laquelle $\mathrm{F}_{i}$ est convexe et possède un feuilletage caractéristique $\xi_{2} \mathrm{~F}_{i}$ scindé par deux courbes, de classe $\pm\left(1, n_{i}-1\right)$. Comme dans la démonstration $\mathrm{du}$ lemme 2.8, les lemmes sur les surfaces convexes permettent ensuite de modifier $\xi_{2}$, par une isotopie toujours relative à $\bigvee_{j \neq i} \mathrm{~F}_{j}$ au cours de laquelle $\mathrm{F}_{i}$ reste convexe, en une structure de contact $\xi_{3}$ tangente aux fibres de $\pi$ sur un voisinage de $\mathrm{F}_{i}$. En renouvelant l'opération, on annule au fur et à mesure les coefficients $n_{i}$. 
Soit $\mathrm{J}_{s}, s \in[0,1]$, une famille lisse d'arcs plongés dans $\mathrm{S} \backslash\left(\operatorname{Int} Q \cup \bigvee_{j \neq i} \mathrm{~K}_{j}\right)$ et ayant les propriétés suivantes :

- $\mathrm{J}_{0}=\mathrm{K}_{i} \backslash \operatorname{Int} Q$ et $\mathrm{J}_{s}$, pour tout $s \in[0,1]$, a un contact d'ordre infini avec $\mathrm{J}_{0}$ en ses extrémités;

- les $\operatorname{arcs} \mathrm{J}_{s}, s \in[0,1]$, sont d'intérieurs disjoints, leur union couvre un voisinage de $\mathrm{D}$ et la trace de chacun d'eux sur Int $\mathrm{D}$ est connexe;

- les anneaux $\mathrm{B}_{s}=\pi^{-1}\left(\mathrm{~J}_{s}\right)$, pour $s>0$, sont du côté positif de $\mathrm{F}_{i}$ - la coorientation provient du paramétrage $\mathbf{T}^{2} \rightarrow \mathrm{F}_{i}$.

Pour tout $s \in[0,1]$, on pose $\mathrm{F}_{i, s}=\left(\mathrm{F}_{i} \backslash \mathrm{B}_{0}\right) \cup \mathrm{B}_{s}$. Le tore $\mathrm{F}_{i, 1}$ est contenu dans $\pi^{-1}(\mathrm{R})$ et les cercles de singularités du feuilletage $\xi_{1} \mathrm{~F}_{i, 1}$ ont pour classe $\pm\left(1, n_{i}+\chi(\mathrm{V}, \mathrm{S})+\chi(\mathrm{S})\right)$. En fait, chaque feuilletage $\xi_{1} \mathrm{~F}_{i, s}$ est tangent aux fibres au-dessus de $J_{s} \backslash \mathrm{D}$ (avec deux singularités par fibre) et transversal aux fibres au-dessus de $\mathrm{J}_{s} \cap \operatorname{Int} \mathrm{D}$. Les singularités de $\xi_{1} \mathrm{~F}_{i, s}$ forment donc deux courbes $\mathrm{C}_{s}^{ \pm}$qui sont fermées si $\mathrm{J}_{s}$ évite Int $\mathrm{D}$ (par exemple pour $s$ proche de 0 et 1 ) mais sont des arcs sinon. Sauf pour un nombre fini de valeurs de $s$ (instants de bifurcation), le tore $\mathrm{F}_{i, s}$ est convexe et les extrémités de l'arc $\mathrm{C}_{s}^{+}$sont reliées par une feuille régulière. La courbe fermée $\overline{\mathrm{C}}_{s}^{+}$, union de cette feuille et de $\mathrm{C}_{s}^{+}$, est parallèle aux courbes qui scindent $\xi_{1} \mathrm{~F}_{i, s}$; sa classe d'homologie, lorsque $s$ varie de 0 à 1 , prend successivement les valeurs $\pm\left(1, n_{i}\right), \pm\left(1, n_{i}-1\right), \ldots$, $\pm\left(1, n_{i}+\chi(\mathrm{V}, \mathrm{S})+\chi(\mathrm{S})\right) \quad$ (la condition de contact impose la décroissance de la pente, comme dans la proposition 1.2).

On choisit désormais pour $s$ un instant où la classe de $\overline{\mathrm{C}}_{s}^{+}$vaut $\pm\left(1, n_{i}-1\right)$. Le feuilletage $\xi_{1} \mathrm{~F}_{i, s}$ est clairement scindé par le bord d'un voisinage annulaire de $\overline{\mathrm{C}}_{s}^{+}$donc $\mathrm{F}_{i, s}$ est convexe. Soit $\phi_{t}, t \in[0, s]$, une isotopie de $\mathrm{V}$ qui prolonge l'isotopie $\mathrm{F}_{i, t}$ sans bouger les points de $\pi^{-1}(Q) \cup \bigvee_{j \neq i} \mathrm{~F}_{j}$. Les structures de contact $\phi_{t}^{*} \xi_{1}$ donnent la déformation voulue entre $\xi_{1}$ et $\xi_{2}=\phi_{s}^{*} \xi_{1}$.

Fort du lemme 3.8, on suppose dorénavant que $\xi_{1}$ coïncide avec $\xi_{0}$ au-dessus de $\mathrm{R}$ et on pose $\sigma=\xi_{i} \partial \mathrm{W}$. On note que, d'après le lemme 2.12, les restrictions de $\xi_{0}$ et $\xi_{1}$ à $\mathrm{W}$ sont universellement tendues. Or, d'après le théorème 1.6 de [Gi4], les structures de contact universellement tendues sur W qui impriment $\sigma$ sur $\partial \mathrm{W}$ forment deux classes d'isotopie relative au bord. Mieux, $\left.\xi_{0}\right|_{\mathrm{w}}$ et $\left.\xi_{1}\right|_{\mathrm{w}}$ sont dans la même classe si et seulement si les anneaux respectifs $\mathrm{A}_{0}$ et $\mathrm{A}_{1}$ que leur attribue le lemme 2.9 - anneaux qui s'appuient sur les cercles de singularités de $\sigma$ - sont isotopes relativement à leur bord (cf. démonstration de la proposition 2.10). Si $A_{1}$ n'est pas isotope à $A_{0}$ relativement à son bord, on amène $A_{1}$ sur $A_{0}$ par une isotopie fibrée de $\mathrm{W}$ qui permute les cercles de singularités de $\sigma$. On prolonge ci-dessous cette isotopie en une isotopie fibrée $\phi_{t}$ de $\mathrm{V}$ dont le stade final $\phi_{1}$ préserve $\left.\xi_{1}\right|_{\pi^{-1}(\mathrm{R})}$. Du coup, $\xi_{0}$ est isotope à $\left(\phi_{1}\right)_{*} \xi_{1}$ relativement à $\pi^{-1}(\mathrm{R})$, donc $\xi_{0}$ et $\xi_{1}$ sont isotopes.

Comme $\mathrm{R}$ est orientable, un avatar de la proposition 3.3 donne un difféomorphisme fibré de $\pi^{-1}(\mathrm{R})$ sur $\mathbf{S}(\mathrm{TR})$ qui envoie $\xi_{1}$ sur la structure de contact canonique $\xi_{\mathrm{R}}$. Moyennant le choix d'une structure conforme sur $\mathrm{R}$, on note $\psi_{t}$ l'isotopie fibrée de $\mathbf{S}(\mathrm{TR})$ qui tourne les droites d'un angle $\pi t, t \in[0,1]$. 
Le difféomorphisme $\psi_{1}$ préserve $\xi_{\mathrm{R}}$ et permute les courbes de singularités du feuilletage $\xi_{\mathrm{R}} \partial \mathbf{S}(\mathrm{TR})$. Par suite, l'isotopie $\psi_{t}$, transportée sur $\pi^{-1}(\mathrm{R})$, fournit le prolongement voulu.

\section{E. Structures d'enroulement serré}

On démontre ici la partie c) du théorème 3.1 , au calcul près du nombre exact des classes de conjugaison qu'on effectue dans la proposition 3.10. On suppose donc que $n \chi(\mathrm{V}, \mathrm{S})=-\chi(\mathrm{S})$, où $n>0$ est entier, et on s'intéresse sur $\mathrm{V}$ aux structures de contact universellement tendues et d'enroulement $-n$. Toute structure de ce type est orientable le long des fibres (car $n$ est entier) et est isotope, d'après la proposition 3.7, à une structure de contact tangente aux fibres. D'autre part, l'avatar orienté de la proposition 3.3 assure que toute structure de contact $\xi$ tangente aux fibres et orientable le long des fibres s'écrit $\rho^{*} \xi_{\mathrm{S}}$, où $\rho$ est un revêtement fibré $\mathrm{V} \rightarrow \mathbf{S}(\mathrm{TS})$ et $\xi_{\mathrm{S}}$ la structure de contact canonique sur $\mathbf{S}(\mathrm{TS})$.

On dira que deux revêtements fibrés $\rho_{0}, \rho_{1}: \mathrm{V} \rightarrow \mathbf{S}(\mathrm{TS})$ sont isomorphes audessus de $\mathrm{S}$ s'il existe des difféomorphismes $\phi$ de $\mathrm{V}$ et $\bar{\phi}$ de $\mathbf{S}(\mathrm{TS})$, fibrés au-dessus d'un difféomorphisme de $\mathrm{S}$, qui rendent commutatif le diagramme

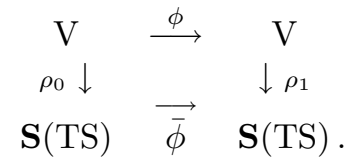

Comme dans la section $\mathrm{D}$, on prend sur $\mathrm{S}$ un bouquet de cercles $\mathrm{K}=\bigvee_{i=1}^{2 g} \mathrm{~K}_{i}$ ayant pour complémentaire un disque et on lisse les lacets $K_{i}$ en des courbes $K_{i}^{\prime}$. On paramètre de nouveau chaque tore $\mathrm{F}_{i}=\pi^{-1}\left(\mathrm{~K}_{i}^{\prime}\right)$ par $\mathbf{T}^{2}$ de telle sorte que les fibres soient les cercles $\{*\} \times \mathbf{S}^{1}$. Pour toute structure de contact $\xi$ sur V tangente aux fibres et d'enroulement $-n$, la courbe des singularités du feuilletage $\xi \mathrm{F}_{i}$ - toutes composantes orientées dans le même sens - a alors une classe d'homologie qui s'écrit

$$
\pm 2\left(n, m_{i}(\xi)\right) \in H_{1}\left(\mathrm{~F}_{i} ; \mathbf{Z}\right) \cong \mathbf{Z}^{2} .
$$

En outre, étant donné des entiers $m_{1}, \ldots, m_{2 g} \in \mathbf{Z}$, on construit sans peine une structure de contact $\xi$ tangente aux fibres et d'enroulement $-n$ pour laquelle $m_{i}=m_{i}(\xi), \quad 1 \leq i \leq 2 g$. Lorsque $\chi(\mathrm{S})<0$, la partie c) du théorème 3.1 découle donc du lemme suivant et de la proposition 3.10 :

Lemme 3.9. Soit $\xi_{0}$ et $\xi_{1}$ deux structures de contact tangentes aux fibres et d'enroulement $-n$.

a) Les structures $\xi_{0}$ et $\xi_{1}$ sont isotopes si et seulement si les entiers $m_{i}\left(\xi_{0}\right)$ et $m_{i}\left(\xi_{1}\right)$ sont égaux pour $1 \leq i \leq 2 g$. 
b) Lorsque $\chi(\mathrm{S})<0$, les structures $\xi_{0}$ et $\xi_{1}$ sont conjuguées si et seulement si les revêtements fibrés associés, de $\mathrm{V}$ sur $\mathbf{S}(\mathrm{TS})$, sont isomorphes au-dessus de $\mathrm{S}$.

Démonstration.

a) Si $m_{i}\left(\xi_{0}\right)=m_{i}\left(\xi_{1}\right)$ pour $1 \leq i \leq 2 g$, une isotopie fibrée permet clairement d'amener $\xi_{0}$ sur $\xi_{1}$. Par ailleurs, si on note $q$ le sommet du bouquet $\mathrm{K}$, chaque lacet $\mathrm{K}_{i}$ engendre dans $\pi_{1}(\mathrm{~S}, q)$ un groupe cyclique infini. Il lui est ainsi associé un revêtement $\rho_{i}: \tilde{\mathrm{S}}_{i} \rightarrow \mathrm{S}$ et on paramètre $\tilde{\mathrm{S}}_{i}$ par $\mathbf{R} \times \mathbf{S}^{1}$ de telle sorte que $\rho\left(\{0\} \times \mathbf{S}^{1}\right)$ soit la courbe $\mathbf{K}_{i}^{\prime}$. Les relèvements respectifs $\tilde{\xi}_{0}$ et $\tilde{\xi}_{1}$ de $\xi_{0}$ et $\xi_{1}$ sur $\tilde{\mathrm{V}}=\rho^{*} \mathrm{~V}$ sont des structures de contact tangentes aux fibres de la fibration $\tilde{\mathrm{V}} \rightarrow \tilde{\mathrm{S}}$. De plus, on peut paramétrer $\tilde{\mathrm{V}}$ par $\mathbf{R} \times \mathbf{T}^{2}$ de telle sorte que les conditions suivantes soient remplies :

- le plongement composé $\mathbf{T}^{2}=\{0\} \times \mathbf{T}^{2} \rightarrow \tilde{\mathrm{V}} \rightarrow \mathrm{V}$ a pour image $\mathrm{F}_{i}$ et coïncide avec le paramétrage donné de $\mathrm{F}_{i}$;

- la fibration $\tilde{\mathrm{V}} \rightarrow \tilde{\mathrm{S}}$ est la projection sur $\mathbf{R} \times \mathbf{S}^{1}$.

Du coup, tous les tores $\mathrm{T}_{a}=\{a\} \times \mathbf{T}_{\tilde{\varepsilon}}^{2}, a \in \mathbf{R}$, sont convexes et la courbe qui scinde leur feuilletage $\tilde{\xi}_{0} \mathrm{~T}_{a}$ (resp. $\left.\quad \tilde{\xi}_{1} \mathrm{~T}_{a}\right)$ a pour classe $\pm\left(1, m_{i}\left(\xi_{0}\right)\right.$ ) (resp. $\left.\pm\left(1, m_{i}\left(\xi_{1}\right)\right)\right)$. Si $m_{i}\left(\xi_{0}\right)$ est différent de $m_{i}\left(\xi_{1}\right)$, le lemme 4.7 de [Gi4] (qui est un cas particulier de l'inégalité de Bennequin semi-locale, cf. proposition 4.10) montre que $\tilde{\xi}_{0}$ n'est pas isotope à $\tilde{\xi}_{1}$. Par suite, $\xi_{0}$ et $\xi_{1}$ ne sont pas isotopes.

b) Soit $\rho_{0}$ et $\rho_{1}$ les revêtements fibrés $\mathrm{V} \rightarrow \mathbf{S}(\mathrm{TS})$ associés respectivement à $\xi_{0}$ et $\xi_{1}$. Si $\rho_{0}$ et $\rho_{1}$ sont isomorphes au-dessus de $\mathrm{S}$, tout difféomorphisme fibré de $\mathrm{V}$ qui les conjugue envoie en même temps $\xi_{0}$ sur $\xi_{1}$. On suppose donc maintenant que $\xi_{0}$ et $\xi_{1}$ sont conjuguées par un difféomorphisme $\phi_{0}$ de $\mathrm{V}$. D'après [Wa], $\phi_{0}$ est isotope à un difféomorphisme fibré $\phi$. La structure de contact $\phi^{*} \xi_{1}$ est alors tangente aux fibres et isotope à $\xi_{0}$. Or il ressort immédiatement du a) que, si deux structures de contact tangentes aux fibres et de même enroulement sont isotopes, elles le sont par une isotopie fibrée. À l'instant final, cette isotopie conjugue $\rho_{0}$ à $\phi^{*} \rho_{1}$, donc $\rho_{0}$ et $\rho_{1}$ sont isomorphes au-dessus de S.

Il reste à regarder le cas (peu intéressant) où $\chi(\mathrm{S})=0$. La relation $n \chi(\mathrm{V}, \mathrm{S})=$ $-\chi(\mathrm{S})$ force alors $\mathrm{V}$ à être un tore. D'après [Gi4] (voir aussi [Ka]), toute structure de contact (universellement) tendue sur $\mathbf{T}^{3}$ est conjuguée à une structure de contact $\zeta_{m}$ d'équation

$$
\cos (m \theta) d x_{1}-\sin (m \theta) d x_{2}=0, \quad m>0, \quad\left(x_{1}, x_{2}, \theta\right) \in \mathbf{T}^{3} .
$$

Pour la projection $\left(x_{1}, x_{2}, \theta\right) \mapsto\left(x_{1}, x_{2}\right)$, la structure $\zeta_{m}$ est tangente aux fibres et d'enroulement $-m$. Mais, pour chaque entier $d>0$, on peut aussi trouver une fibration $\mathbf{T}^{3} \rightarrow \mathbf{T}^{2}$ pour laquelle $\zeta_{m}$ est d'enroulement $-d m$. 


\section{F. Dénombrement des revêtements fibrés}

On classifie ici les revêtements fibrés de $\mathbf{S}(\mathrm{TS})$ à isomorphisme près au-dessus de $\mathrm{S}$, ce qui complète la démonstration du théorème 3.1-c.

Proposition 3.10. Soit S une surface close orientable, de caractéristique d'Euler négative ou nulle et soit $n$ un entier positif.

a) $\grave{A}$ isomorphisme près au-dessus de $\mathrm{S}$, les revêtements fibrés de $\mathbf{S}(\mathrm{TS}) \grave{a}$ $n$ feuillets sont classés par le quotient $H^{1}(\mathrm{~S} ; \mathbf{Z} / n \mathbf{Z}) / \operatorname{Aut} \pi_{1}(\mathrm{~S})$.

b) À composition près par les automorphismes de $\pi_{1}(\mathrm{~S})$, les morphismes de $\pi_{1}(\mathrm{~S})$ dans $\mathbf{Z} / n \mathbf{Z}$ sont classés par leur image. Le quotient $H^{1}(\mathrm{~S} ; \mathbf{Z} / n \mathbf{Z}) /$ Aut $\pi_{1}(\mathrm{~S})$ a donc un cardinal égal au nombre de diviseurs de $n$.

Démonstration.

a) On pose $V_{0}=\mathbf{S}(\mathrm{TS})$ et on choisit dans $\mathrm{V}_{0}$ un point de référence. Comme $\chi(\mathrm{S}) \leq 0$, le groupe fondamental $\pi_{1}\left(\mathrm{~V}_{0}\right)$ est une extension centrale de $\pi_{1}(\mathrm{~S})$ par $\mathbf{Z} \cong \pi_{1}\left(\mathbf{S}^{1}\right)$. La suite exacte courte d'homotopie $\mathbf{Z} \rightarrow \pi_{1}\left(\mathrm{~V}_{0}\right) \rightarrow \pi_{1}(\mathrm{~S})$ induit ainsi une suite exacte courte d'homologie $\mathbf{Z} / \chi(\mathrm{S}) \mathbf{Z} \rightarrow H_{1}\left(\mathrm{~V}_{0} ; \mathbf{Z}\right) \rightarrow H_{1}(\mathrm{~S})$.

D'autre part, la bijection entre les (classes d'équivalence de) revêtements de $V_{0}$ et les (classes de conjugaison de) sous-groupes de $\pi_{1}\left(\mathrm{~V}_{0}\right)$ associe aux revêtements fibrés de degré $n$ les sous-groupes d'indice $n$ dont la trace sur le sous-groupe central $\mathbf{Z}$ est $n \mathbf{Z}$. Ces sous-groupes sont normaux et sont donc les noyaux des morphismes $\pi_{1}\left(\mathrm{~V}_{0}\right) \rightarrow \mathbf{Z} / n \mathbf{Z}$ dont la restriction à $\mathbf{Z}$ est la projection canonique. Comme de tels morphismes transitent par $H_{1}\left(\mathrm{~V}_{0} ; \mathbf{Z}\right)$, ils existent dès que $n$ divise $\chi(\mathrm{S})$ et forment alors un espace principal homogène du groupe $H^{1}(\mathrm{~S} ; \mathbf{Z} / n \mathbf{Z})$. En outre, l'action des difféomorphismes fibrés de $\mathrm{V}_{0}$ se réduit sur $H^{1}(\mathrm{~S} ; \mathbf{Z} / n \mathbf{Z})$ à l'action des difféomorphismes de $\mathrm{S}$, i.e. des automorphismes (extérieurs) de $\pi_{1}(\mathrm{~S})$.

b) Soit $f_{0}$ et $f_{1}$ des morphismes de $\pi_{1}(\mathrm{~S})$ dans $\mathbf{Z} / n \mathbf{Z}$. Comme $\pi_{1}(\mathrm{~S})$ est sans torsion, $f_{0}$ et $f_{1}$ se relèvent en des morphismes $\tilde{f}_{0}$ et $\tilde{f}_{1}$ de $\pi_{1}(\mathrm{~S})$ dans $\mathbf{Z}$. Ceux-ci sont en dualité de Poincaré avec des éléments $m_{0} u_{0}$ et $m_{1} u_{1}$ de $H_{1}(\mathrm{~S} ; \mathbf{Z})$, où $m_{0}, m_{1}$ sont des entiers et $u_{0}, u_{1}$ des classes primitives. On se donne alors des courbes fermées simples $\mathrm{A}_{0}$ et $\mathrm{A}_{1}$ dont les classes d'homologie respectives sont $u_{0}$ et $u_{1}$. Comme il existe un difféomorphisme $\phi$ de $\mathrm{S}$ qui envoie $\mathrm{A}_{0}$ sur $\mathrm{A}_{1}$, on peut supposer, quitte à composer $f_{1}$ par $\phi_{*}$, que $\mathrm{A}_{0}=\mathrm{A}_{1}=\mathrm{A}$. Le morphisme $f_{i}$ associe alors à la classe de chaque courbe $\mathrm{C}$ le nombre $m_{i}[\mathrm{~A}] \cdot[\mathrm{C}](\bmod n)$. Par suite, $f_{0}$ et $f_{1}$ ont même image dans $\mathbf{Z} / n \mathbf{Z}$ si et seulement si $m_{0} \mathbf{Z}+n \mathbf{Z}=$ $m_{1} \mathbf{Z}+n \mathbf{Z}$, c'est-à-dire si et seulement si $\operatorname{pgcd}\left(m_{0}, n\right)=\operatorname{pgcd}\left(m_{1}, n\right)$. On suppose que c'est le cas, on note $d$ ce plus grand commun diviseur et on pose $m_{i}=d m_{i}^{\prime}$, $n=d n^{\prime}$. On choisit dans $\mathrm{S}$ une sous-surface compacte $\mathrm{R}$ contenant $\mathrm{A}$ et difféomorphe à un tore troué. On note $\mathrm{B} \subset \mathrm{R}$ une courbe fermée simple qui, avec A, forme une base de $H_{1}(\mathrm{R} ; \mathbf{Z})$. Modulo $n$, l'intersection de $m_{i}[\mathrm{~A}]$ avec la classe d'une courbe quelconque $\mathrm{C}$ de $\mathrm{S}$ est égale à celle de $m_{i}[\mathrm{~A}]+n[\mathrm{~B}]$ avec $[\mathrm{C}]$. Or 
$m_{i}[\mathrm{~A}]+n[\mathrm{~B}]=d\left(m_{i}^{\prime}[\mathrm{A}]+n^{\prime}[\mathrm{B}]\right)$ et, comme $\operatorname{pgcd}\left(m_{0}^{\prime}, n^{\prime}\right)=\operatorname{pgcd}\left(m_{1}^{\prime}, n^{\prime}\right)=1$, il existe un difféomorphisme $\psi$ de $\mathrm{S}$, à support dans $\mathrm{R}$, qui envoie $m_{0}^{\prime}[\mathrm{A}]+n^{\prime}[\mathrm{B}]$ sur $m_{1}^{\prime}[\mathrm{A}]+n^{\prime}[\mathrm{B}]$. Ainsi, $f_{0}=f_{1} \circ \psi_{*}$.

\section{4. Étude des structures de contact non transversales}

\section{A. Structures invariantes}

On considère ici une variété $\mathrm{V}$ close, connexe et orientée, munie d'une action libre du cercle $\mathbf{S}^{1}$ et ainsi fibrée au-dessus de la surface quotient $\mathrm{S}=\mathrm{V} / \mathbf{S}^{1}$. On s'intéresse sur $\mathrm{V}$ aux structures de contact invariantes par l'action. Pour une telle structure $\xi$, on note $\Gamma(\xi)$ l'ensemble des orbites $q \in \mathrm{S}$ qui sont tangentes à $\xi$ (i.e. legendriennes). Il est facile de voir que $\Gamma(\xi)$ est une multi-courbe lisse sur $\mathrm{S}$ et R. Lutz montre dans [Lu] que deux structures invariantes $\xi_{0}$ et $\xi_{1}$ sont conjuguées par un difféomorphisme équivariant de $\mathrm{V}$ si et seulement s'il existe un difféomorphisme de $\mathrm{S}$ qui envoie $\Gamma\left(\xi_{0}\right)$ sur $\Gamma\left(\xi_{1}\right)$. Avant d'expliquer comment affranchir ces résultats des conditions d'invariance et d'équivariance, on établit une caractérisation des structures invariantes (universellement) tendues.

Proposition 4.1. Soit $\xi$ une structure de contact orientable et invariante sur V . a) Si $\xi$ est tendue et si une composante connexe de $\mathrm{S} \backslash \Gamma(\xi)$ est un disque, $\Gamma(\xi)$ est connexe et $\chi(\mathrm{V}, \mathrm{S})$ vérifie l'inégalité

$$
\left\{\begin{array}{l}
\chi(\mathrm{V}, \mathrm{S})>0 \quad \text { si } \mathrm{S} \neq \mathrm{S}^{2} \\
\chi(\mathrm{V}, \mathrm{S}) \geq 0 \quad \text { si } \mathrm{S}=\mathbf{S}^{2}
\end{array}\right.
$$

b) Pour que $\xi$ soit universellement tendue, il faut et il suffit que l'une des conditions suivantes soit remplie:

- $\mathbf{S} \nsucceq \mathbf{S}^{2}$ et aucune composante connexe de $\mathbf{S} \backslash \Gamma(\xi)$ n'est un disque;

- $\quad \mathbf{S} \simeq \mathbf{S}^{2}, \quad \chi(\mathrm{V}, \mathrm{S})<0$ et $\Gamma(\xi)$ est vide;

- $\quad \mathrm{S} \simeq \mathrm{S}^{2}, \quad \chi(\mathrm{V}, \mathrm{S}) \geq 0$ et $\Gamma(\xi)$ est connexe mais pas vide.

Démonstration.

a) Soit $\mathrm{D}$ une composante de $\mathrm{S} \backslash \Gamma(\xi)$ qui est un disque et $\mathrm{E}$ la composante voisine. Si $\Gamma(\xi)$ n'est pas connexe, la surface compacte $\mathrm{R}=\operatorname{Adh}(\mathrm{D} \cup \mathrm{E})$ diffère de $\mathrm{S}$ et la fibration $\pi: \mathrm{V} \rightarrow \mathrm{S}$ admet une section $\hat{\mathrm{R}}$ au-dessus de $\mathrm{R}$ ayant, pour un choix convenable d'orientations, les propriétés suivantes :

1) la courbe $\partial \hat{\mathrm{R}}$ est positivement transversale à $\xi$;

2) la surface $\hat{R}$ a un seul point de contact négatif avec $\xi$ - situé dans $D$ - et ce point est une singularité d'indice 1 du feuilletage $\xi \hat{\mathrm{R}}$.

Or l'inégalité de Bennequin [El2] interdit l'existence d'une telle surface $\hat{\mathrm{R}}$ si $\xi$ est tendue.

On établit maintenant l'inégalité sur le nombre d'Euler. Si $\chi(\mathrm{V}, \mathrm{S})<0$ et si $Q \subset \mathrm{S} \backslash \mathrm{D}$ est un disque assez petit, la fibration $\pi$ admet sur $\mathrm{R}=\mathrm{S} \backslash \operatorname{Int} Q$ une 
section $\hat{\mathrm{R}}$ ayant elle aussi, pour un choix convenable d'orientations, les propriétés 1 ) et 2) ci-dessus qui sont illusoires si $\xi$ est tendue. Enfin, si $\chi(V, S)=0$ et si $\mathrm{S} \neq \mathbf{S}^{2}$, le lemme 2.6 de [Gi2] (qui sert à établir l'inégalité de Bennequin relative aux surfaces closes) montre encore que $\xi$ est vrillée.

b) Pour voir que l'une des conditions énumérées est remplie quand $\xi$ est universellement tendue, il suffit d'appliquer a) et d'observer que, si $\mathbf{S} \nsucceq \mathbf{S}^{2}$ et si une composante connexe de $\mathrm{S} \backslash \Gamma(\xi)$ est un disque, l'image inverse de $\Gamma(\xi)$ par n'importe quel revêtement non trivial $\rho: \tilde{\mathrm{S}} \rightarrow \mathrm{S}$ est non connexe. Or cette multicourbe n'est autre que $\Gamma(\tilde{\xi})$ où $\tilde{\xi}$ désigne le rappel de $\xi$ sur $\tilde{V}=\rho^{*} \mathrm{~V}$. Ainsi, $\tilde{\xi}$ est vrillée et $\xi$ l'est virtuellement.

On explique maintenant pourquoi $\xi$ est universellement tendue lorsque $\Gamma(\xi)$ satisfait l'une des conditions requises.

Si $\mathbf{S}=\mathbf{S}^{2}$, la classification de Lutz montre que le revêtement universel $(\tilde{\mathrm{V}}, \tilde{\xi})$ de $(\mathrm{V}, \xi)$ est fait comme suit, à un isomorphisme près :

- si $\chi(\mathrm{V}, \mathrm{S})=0$, alors $\tilde{\mathrm{V}}=\mathbf{S}^{2} \times \mathbf{R}=\mathbf{R}^{3} \backslash\{0\}$ et $\tilde{\xi}$ est la structure usuelle, d'équation $d z+x d y-y d x=0$, qui est invariante par l'action du flot $(x, y, z) \mapsto\left(e^{t} x, e^{t} y, e^{2 t} z\right)$;

- si $\chi(\mathrm{V}, \mathrm{S})=\mp 1$, alors $\tilde{\mathrm{V}}=\mathbf{S}^{3}$ est la sphère unité de $\mathbf{C}^{2}$ et $\tilde{\xi}$ est la structure usuelle, d'équation $\operatorname{Im}(\bar{z} d z+\bar{w} d w)=0$, qui est invariante par l'action du flot $(z, w) \mapsto\left(e^{i \theta} z, e^{ \pm i \theta} w\right)$.

Dans tous ces cas, le théorème de Bennequin assure que $\tilde{\xi}$ est tendue.

Si $\mathbf{S} \neq \mathbf{S}^{2}$, le revêtement universel de $\mathbf{S}$ est $\mathbf{R}^{2}$ et il suffit de voir que la structure $\tilde{\xi}$ induite par $\xi$ sur $\tilde{\mathrm{V}}=\mathbf{R}^{2} \times \mathbf{S}^{1}$ est tendue. Comme toutes les composantes de $\Gamma(\xi)$ sont essentielles sur S (i.e. non contractiles), celles de $\Gamma(\tilde{\xi})$ sont des droites proprement plongées dans $\mathbf{R}^{2}$. On remplit $\mathbf{R}^{2}$ avec une suite exhaustive de disques fermés $\mathrm{D}_{n}$ dont les bords sont transversaux à $\Gamma(\tilde{\xi})$. On va montrer que $\tilde{\xi}$ est tendue en plongeant chaque domaine $\left(\mathrm{D}_{n} \times \mathbf{S}^{1}, \tilde{\xi}\right)$ dans $\left(\mathbf{S}^{2} \times \mathbf{S}^{1}, \eta\right)$ où $\eta$ est une structure de contact invariante ayant une courbe $\Gamma(\eta)$ connexe.

On se donne des équations invariantes de $\tilde{\xi}$ et $\eta$ qu'on écrit respectivement $\beta+u d t=0$ et $\lambda+v d t=0$, où $t \in \mathbf{S}^{1}$ et où $\beta, u$ (resp. $\lambda, v$ ) sont une 1 -forme et une fonction sur $\mathbf{R}^{2}$ (resp. sur $\mathbf{S}^{2}$ ). Les ensembles $\Gamma(\tilde{\xi})$ et $\Gamma(\eta)$ ont donc pour équations respectives $u=0$ et $v=0$. On choisit, pour tout $n \geq 0$, un plongement $\phi_{n}: \mathrm{D}_{n} \rightarrow \mathbf{S}^{2}$ qui envoie $\Gamma(\tilde{\xi}) \cap \mathrm{D}_{n}$ sur $\Gamma(\eta) \cap \phi_{n}\left(\mathrm{D}_{n}\right)$ en respectant les coorientations induites par $u$ et $v$. Il existe ainsi une fonction $\left.h_{n}: \mathrm{D}_{n} \rightarrow\right] 0, \infty\left[\right.$ telle que $v \circ \phi_{n}=h_{n} u$ et on pose $\beta_{n}=h_{n} \beta$. Le lemme 4.2 ci-dessous garantit alors que la forme $\left(\phi_{n}\right)_{*} \beta_{n}$ se prolonge à $\mathbf{S}^{2}$ en une forme $\lambda_{n}$ vérifiant l'inégalité $v d \lambda_{n}+\lambda_{n} \wedge d v>0$, laquelle assure que l'équation $\lambda_{n}+v d t=0$ définit une structure de contact $\eta_{n}$ invariante sur $\mathbf{S}^{2} \times \mathbf{S}^{1}$. Or, par construction,

$$
\phi_{n} \times \mathrm{id}:\left(\mathrm{D}_{n} \times \mathbf{S}^{1}, \tilde{\xi}\right) \longrightarrow\left(\mathbf{S}^{2} \times \mathbf{S}^{1}, \eta_{n}\right)
$$

est un plongement de contact et, comme $\Gamma\left(\eta_{n}\right)=\{v=0\}=\Gamma(\eta)$, la structure $\eta_{n}$ est isotope à $\eta$. Par conséquent, la structure $\tilde{\xi}$ est tendue et $\xi$ l'est 
universellement.

Lemme 4.2. Soit $\mathrm{S}$ une surface compacte orientée, $\mathrm{R}$ une sous-surface compacte et $v: \mathrm{S} \rightarrow \mathbf{R}$ une fonction qui admet 0 pour valeur régulière, de même que $\left.v\right|_{\partial \mathrm{S}}$ et $\left.v\right|_{\partial \mathrm{R}}$. Si $v$ s'annule dans chaque composante de $\mathrm{S} \backslash \mathrm{R}$, toute 1 -forme $\lambda$ sur $\mathrm{R}$ qui satisfait à l'inégalité

$$
v d \lambda+\lambda \wedge d v>0
$$

se prolonge à $\mathrm{S}$ en une 1 -forme vérifiant partout l'inégalité (4.2).

Démonstration. En un point de $\Gamma=\{v=0\}$, l'inégalité (4.2) dit simplement que $\lambda$ est transversale à $d v$. On prolonge donc sans peine $\lambda$ à un voisinage $U$ de $\Gamma$. D'autre part, en tout point de $\mathrm{S} \backslash \Gamma$,

$$
v d \lambda+\lambda \wedge d v=v^{2} d(\lambda / v) .
$$

On observe alors que, par hypothèse, chaque composante $\mathrm{D}$ de $\mathrm{S} \backslash(\mathrm{R} \cup \Gamma)$ contient au moins un arc $\mathrm{J}$ de $\Gamma$ dans sa frontière. Par suite, l'intégrale de $\lambda / v$ sur le bord de $\mathrm{D}$ est infinie. Quitte à diminuer le voisinage $U$, on peut donc prolonger $\lambda / v$ à $\mathrm{D}$ en une 1 -forme dont la différentielle extérieure soit partout positive.

\section{B. Comment revisiter Lutz}

On donne ici une description de toutes les structures de contact universellement tendues sur une variété fibrée en cercles au-dessus d'une surface.

Définition 4.3. Soit V une variété orientée fibrée en cercles au-dessus d'une surface compacte $\mathrm{S}$. Une multi-courbe sur $\mathrm{S}$ est ici une union disjointe d'un nombre fini de courbes fermées simples et d'arcs proprement plongés dans S. Par ailleurs, une multi-courbe est essentielle si aucune de ses composantes n'est nulle en homotopie - relative au bord s'il s'agit d'un arc.

On dira qu'une structure de contact $\xi$ sur $\mathrm{V}$ est cloisonnée par une multicourbe $\Gamma \subset \mathrm{S}$ si les conditions suivantes sont remplies:

- $\operatorname{sur} \mathrm{V} \backslash \pi^{-1}(\Gamma)$, la structure $\xi$ est transversale aux fibres;

- la surface $\pi^{-1}(\Gamma)$ est transversale à $\xi$ et ses caractéristiques sont des fibres.

Exemple. Toute variété orientée $\mathrm{V}$ fibrée en cercles au-dessus d'une surface $\mathrm{S}$ (orientable) peut être munie d'une action libre du cercle qui définit la fibration. Quand S est compacte, R. Lutz construit dans [Lu], pour toute multi-courbe non vide $\Gamma$ dans $\mathrm{S}$, une structure de contact invariante $\xi$ sur V telle que $\Gamma(\xi)$ soit égal à $\Gamma$. Cette structure est alors cloisonnée par $\Gamma$. En outre, elle est orientable si et seulement si la classe de $\Gamma$ dans $H_{1}(\mathrm{~S} ; \mathbf{Z} / 2 \mathbf{Z})$ est nulle. 
Un théorème de recollement dû à V. Colin [Co] assure que toute structure de contact cloisonnée par une multi-courbe essentielle est universellement tendue. ${ }^{4}$ Réciproquement :

Théorème 4.4. Soit V une variété connexe et orientée, fibrée en cercles audessus d'une surface close $\mathrm{S}$ de caractéristique d'Euler négative ou nulle.

a) Toute structure de contact orientable et universellement tendue est isotope à une structure cloisonnée par une multi-courbe essentielle.

b) Soit $\xi_{0}$ et $\xi_{1}$ des structures de contact cloisonnées par des multi-courbes essentielles non vides, respectivement notés $\Gamma_{0}$ et $\Gamma_{1}$. Les structures $\xi_{0}$ et $\xi_{1}$ sont isotopes si et seulement si les multi-courbes $\Gamma_{0}$ et $\Gamma_{1}$ le sont.

Avec les théorèmes 3.1 et 2.3 - ce dernier montrant en particulier qu'une structure transversale aux fibres ne peut être isotope à une structure cloisonnée par une multi-courbe non vide -, le théorème ci-dessus établit une classification des structures de contact universellement tendues sur $\mathrm{V}$ lorsque la caractéristique d'Euler de S est négative ou nulle. Pour les variétés fibrées en cercles au-dessus de la sphère, qui sont des espaces lenticulaires, le théorème 1.1 de [Gi4] donne une classification de toutes les structures de contact tendues.

D'autre part, la partie a) du théorème 4.4 prouve que toute structure de contact universellement tendue et d'enroulement positif ou nul est isotope à une structure invariante (par une quelconque action libre du cercle qui définit la fibration). La partie b) classifie donc en fait les structures de contact $\mathbf{S}^{1}$-invariantes. Sa démonstration s'adapte alors, sans surprise, aux structures de contact $\mathbf{R}$ invariantes sur le produit d'une surface par $\mathbf{R}$. On obtient ainsi, compte tenu de l'abondance des surfaces convexes (cf. section 2.D), une classification " générique » des structures de contact tendues au voisinage des surfaces :

Théorème 4.5. Soit $(\mathrm{M}, \xi)$ une variété de contact de dimension $3, \mathrm{~F} \subset(\mathrm{M}, \xi)$ une surface convexe close, $U=\mathrm{F} \times \mathbf{R}$ un voisinage homogène de $\mathrm{F}$ et $\Gamma$ la multi-courbe qui scinde $\xi \mathrm{F}$.

a) La restriction de $\xi$ à $U$ est tendue si et seulement si l'une des conditions suivantes est remplie:

- $\mathrm{F} \nsucceq \mathbf{S}^{2}$ et aucune composante de $\mathrm{F} \backslash \Gamma$ n'est un disque ;

- $\mathrm{F} \simeq \mathbf{S}^{2}$ et $\Gamma$ est connexe mais pas vide.

b) On suppose que $\xi$ est tendue. Une surface convexe $\mathrm{F}^{\prime} \subset(\mathrm{M}, \xi)$ possède un voisinage homogène isomorphe à $(U, \xi)$ si et seulement s'il existe un difféomorphisme de $\mathrm{F}$ dans $\mathrm{F}^{\prime}$ qui envoie $\Gamma$ sur une multi-courbe qui scinde le feuilletage caractéristique $\xi \mathrm{F}^{\prime}$.

La partie a) de ce théorème est un corollaire immédiat de la proposition 4.1. En effet, la restriction de $\xi$ à $U=\mathrm{F} \times \mathbf{R}$ est tendue si et seulement si la structure de contact $\bar{\xi}$ induite par $\xi$ sur $\mathrm{F} \times \mathbf{R} / n \mathbf{Z}$ est tendue pour tout entier $n>0$.

\footnotetext{
4 Une autre démonstration de ce fait s'ensuit de la proposition 4.1 et de la partie b) du théorème 4.4 .
} 
En outre, la multi-courbe $\Gamma_{U}$ (cf. définition 2.6) qui scinde le feuilletage $\xi \mathrm{F}$ n'est autre que $\Gamma(\bar{\xi})$. La partie b) sera démontrée dans la section D.

\section{Existence d'un cloisonnement}

On démontre ici la partie a) du théorème 4.4. La surface $\mathrm{S}$ est donc de caractéristique d'Euler négative ou nulle et les structures de contact qu'on considère sont orientables.

Lemme 4.6. Soit $\xi$ une structure de contact tendue sur $\mathrm{V}$ et $\mathrm{R} \subset \mathrm{S}$ une surface compacte, connexe et à bord non vide. Si l'enroulement $\mathrm{e}(\xi)$ de $\xi$ est positif ou nul, $\xi$ est isotope à une structure qui, au-dessus de $\mathrm{R}$, est cloisonnée par un système d'arcs.

Démonstration. On adapte la démonstration du lemme 2.8. Puisque l'enroulement $\mathrm{e}(\xi)$ est positif ou nul, il existe dans $\mathrm{V}$ une courbe legendrienne isotope à la fibre et d'enroulement nul (proposition 2.4). On regarde alors $\mathrm{R}$ comme un voisinage régulier d'un bouquet de cercles $\mathrm{K}$ de sommet $q$. Quitte à faire une première isotopie, on peut trouver des coordonnées $(x, y, t) \in \mathbf{D}^{2} \times \mathbf{S}^{1}$, au-dessus d'un voisinage compact $Q$ de $q$, dans lesquelles $\pi$ est la projection sur le disque, $\xi$ a pour équation $d y+x d t=0$ et $q=(0,0)$. Sur $N=\pi^{-1}(Q)$, la structure $\xi$ est cloisonnée par l'arc $\mathrm{J}=\{x=0\}$.

On modifie maintenant $K$, par des mouvements de Whitehead à support dans $Q$, en un graphe $\mathrm{K}^{\prime}$ constitué de cercles lisses $\mathrm{K}_{i}^{\prime}, 1 \leq i \leq k$, et d'un arbre $\mathrm{K}_{0}^{\prime}$ inclus dans $Q$. On met en outre tous les sommets de $\mathrm{K}^{\prime}$ sur l'arc
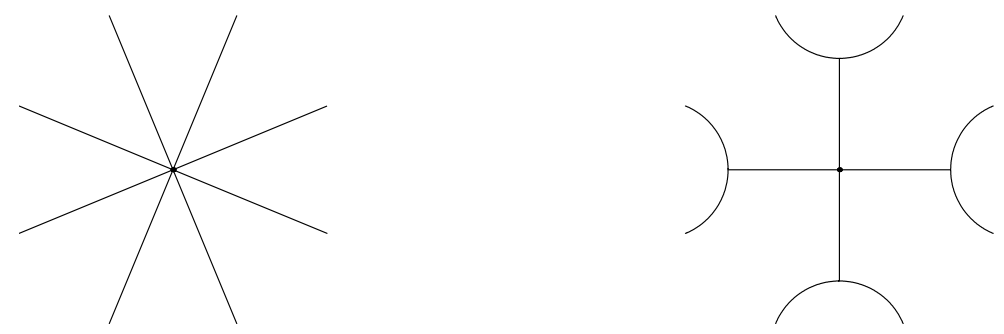

Figure 2. Modification dans $Q$ du bouquet $\mathrm{K}$ en un graphe $\mathrm{K}^{\prime}$ constitué de cercles lisses et d'un petit arbre.

$\mathrm{J} \subset Q$ et on rend les arêtes transversales d'une part à $\mathrm{J}$, d'autre part au vecteur $\partial_{y}$ en tout point d'intersection avec $\mathrm{J}$. On déforme ensuite $\xi$, par une petite isotopie relative à $N$, pour rendre convexe la surface $\pi^{-1}\left(\mathrm{~K}^{\prime} \backslash \operatorname{Int} Q\right)$, qui est une union d'anneaux. Chaque tore $\mathrm{F}_{i}=\pi^{-1}\left(\mathrm{~K}_{i}^{\prime}\right), 1 \leq i \leq k$, est ainsi convexe et la multi-courbe qui scinde son feuilletage caractéristique $\xi \mathrm{F}_{i}$ est verticale. Elle ne 
peut en effet intersecter les fibres au-dessus de $\mathrm{K}_{i}^{\prime} \cap \mathrm{J}$ qui sont des feuilles fermées de $\xi \mathrm{F}_{i}$. On considère alors sur $\mathrm{F}_{i}$ un feuilletage singulier $\sigma_{i}$ ayant les propriétés suivantes :

- $\sigma_{i}$ est scindé par la même multi-courbe que $\xi \mathrm{F}_{i}$;

- $\sigma_{i}$ coïncide avec $\xi \mathrm{F}_{i}$ dans $\mathrm{F}_{i} \cap \pi^{-1}(Q)$;

- $\sigma_{i}$ est non singulier et est transversal aux fibres en dehors de ses feuilles fermées, lesquelles sont des fibres.

Le lemme 2.7 fournit, pour $1 \leq i \leq k$, un plongement $\phi_{i}$ de $\mathrm{F}_{i}$ dans $\mathrm{V}$ - isotope à l'inclusion - dont l'image a pour feuilletage caractéristique $\left(\phi_{i}\right)_{*} \sigma_{i}$ et a même intersection que $\mathrm{F}_{i}$ avec le compact

$$
\mathrm{P}_{i}=\pi^{-1}\left(\mathrm{~J} \cup \mathrm{K}^{\prime \prime}\right) \cup \phi_{1}\left(\mathrm{~F}_{1}\right) \cup \cdots \cup \phi_{i-1}\left(\mathrm{~F}_{i-1}\right) \cup \mathrm{F}_{i+1} \cup \cdots \cup \mathrm{F}_{k} .
$$

Si $\phi$ est un difféomorphisme de $\mathrm{V}$ isotope à l'identité qui prolonge les divers plongements $\phi_{i}$, la structure de contact $\xi^{\prime}=\phi^{*} \xi$ trace sur chaque tore $\mathrm{F}_{i}$ le feuilletage $\sigma_{i}$.

Pour compléter la preuve, on paramètre un voisinage tubulaire de $\mathrm{K}_{i}^{\prime}$ par $\mathrm{K}_{i}^{\prime} \times[-1,1]$, où $\mathrm{K}_{i}^{\prime}=\mathrm{K}_{i}^{\prime} \times\{0\}$. Les tores $\mathrm{F}_{i, s}=\pi^{-1}\left(\mathrm{~K}_{i}^{\prime} \times\{s\}\right)$ ont, pour $s$ petit, un feuilletage $\xi^{\prime} \mathrm{F}_{i, s}$ conjugué à $\xi^{\prime} \mathrm{F}_{i}$ car ce feuilletage est topologiquement stable. On peut donc redresser $\xi^{\prime}$, par une petite isotopie stationnaire sur $\bigcup \mathrm{F}_{i} \cup \pi^{-1}(Q)$, en une structure $\xi^{\prime \prime}$ pour laquelle $\xi^{\prime \prime} \mathrm{F}_{i, s}$ est transversal aux fibres en dehors de ses feuilles fermées, lesquelles sont des fibres. Les projections sur $\mathrm{S}$ des feuilles fermées de tous les feuilletages $\xi^{\prime \prime} \mathrm{F}_{i, s}$, avec $s$ petit, forment alors un système d'arcs qui, avec J, cloisonne $\xi^{\prime \prime}$ sur un voisinage de $\mathrm{K}^{\prime}$. Comme $\mathrm{R}$ se rétracte par isotopie sur un voisinage arbitrairement petit de $\mathrm{K}^{\prime}$, le lemme est démontré.

Désormais, $\xi$ désigne une structure de contact universellement tendue sur $\mathrm{V}$. On note $\mathrm{A}$ un anneau non séparant dans $\mathrm{S}$ et on pose $\mathrm{R}=\mathrm{S} \backslash$ Int $\mathrm{A}$. Compte tenu du lemme ci-dessus, on suppose que $\xi$ est cloisonnée, au-dessus de $\mathrm{R}$, par un système d'arcs $\Gamma_{R} \subset R$. On paramètre $A$ par $\mathbf{S}^{1} \times[0,1]$ et $W=\pi^{-1}(A)$ par $\mathbf{T}^{2} \times[0,1]$ de telle sorte que la fibration $\left.\pi\right|_{\mathrm{W}}$ soit la projection. Pour tout $a \in[0,1]$, on pose encore $\mathrm{T}_{a}=\mathbf{T}^{2} \times\{a\}$. L'argument utilisé au lemme 2.12 montre ici que la restriction de $\xi$ à $\mathrm{W}$ est universellement tendue. D'après les propositions $3.15,3.22$ et 3.29 de [Gi4], la structure $\xi \mid w$ est alors isotope, relativement au bord de $\mathrm{W}$, à une structure de contact $\eta$ pour laquelle il existe dans $\mathrm{A} \simeq \mathbf{S}^{1} \times[0,1]$ une multi-courbe $\Gamma_{\mathrm{A}}$ ayant les propriétés suivantes :

- si $\mathrm{T}_{a} \cap \pi^{-1}\left(\Gamma_{\mathrm{A}}\right) \neq \varnothing$, cette intersection est l'union des feuilles fermées et des singularités de $\eta \mathrm{T}_{a}$ (ces singularités formant donc des courbes);

- si $\mathrm{T}_{a} \cap \pi^{-1}\left(\Gamma_{\mathrm{A}}\right)=\varnothing$, le feuilletage $\eta \mathrm{T}_{a}$ est une suspension dont aucune feuille fermée n'est isotope à la fibre.

À partir de là, on déforme facilement $\eta$, par une isotopie relative à $\partial \mathrm{W} \cup \pi^{-1}\left(\Gamma_{\mathrm{A}}\right)$, en une structure de contact $\eta^{\prime}$ cloisonnée par $\Gamma_{\mathrm{A}}$. En recollant $\eta^{\prime}$ avec la restriction de $\xi$ à $\pi^{-1}(\mathrm{R})$, on obtient une structure de contact $\xi^{\prime}$ cloisonnée par $\Gamma=\Gamma_{\mathrm{R}} \cup \Gamma_{\mathrm{A}}$. Il reste à montrer que les courbes de $\Gamma$ sont toutes essentielles, fait qui résulte de la proposition 4.1 et du lemme suivant : 
Lemme 4.7. Deux structures de contact cloisonnées par une même multi-courbe non vide sont isotopes.

Démonstration. Soit $\xi_{0}$ et $\xi_{1}$ les structures de contact, $\Gamma$ la multi-courbe qui les cloisonne et $u: \mathrm{S} \rightarrow \mathbf{R}$ une fonction dont le niveau $\{u=0\}$ est régulier et égal à $\Gamma$. D'après [Lu] (voir aussi [Gi1]), il existe sur $\mathrm{S}$ une 1 -forme $\beta$ pour laquelle la 2 -forme $u d \beta+\beta \wedge d u$ est une forme d'aire sur $\mathrm{S}$.

On munit maintenant $\mathrm{V}$ d'une action libre du cercle définissant la fibration et d'une forme de connexion $\tau$. Comme chacune des structures $\xi_{i}, i \in\{0,1\}$, est cloisonnée par $\Gamma$, elle admet une équation du type $\beta_{i}+\pi^{*} u \tau=0$ où $\beta_{i}$ est une 1 -forme sur $\mathrm{V}$ nulle sur les vecteurs tangents aux fibres. Un calcul direct montre alors que, si $s$ est un réel positif pris assez grand, les équations de Pfaff

$$
(1-t) \beta_{i}+t s \pi^{*} \beta+\pi^{*} u \tau=0, \quad i \in\{0,1\},
$$

définissent des structures de contact pour tout $t \in[0,1]$. Le théorème de Gray assure dès lors que $\xi_{0}$ et $\xi_{1}$ sont isotopes.

\section{Inégalité de Bennequin semi-locale}

On démontre ici la partie b) du théorème 4.5. S'il existe un difféomorphisme de $\mathrm{F}$ dans $\mathrm{F}^{\prime}$ envoyant $\Gamma$ sur une multi-courbe qui scinde $\xi \mathrm{F}^{\prime}$, les résultats de $[\mathrm{Lu}]$ assurent que tout voisinage homogène de $\mathrm{F}^{\prime}$ est isomorphe à $(U, \xi)$. Pour établir la réciproque, on utilise la notion d'intersection géométrique.

Définition 4.8. Sur une surface close, on considère une courbe fermée simple C et une multi-courbe $\Gamma$. L'intersection géométrique $\mathrm{i}(\Gamma, \mathrm{C})$ est le nombre minimal de points d'intersection entre $\Gamma$ et une courbe quelconque isotope à $\mathrm{C}$.

Un des intérêts de cette notion réside dans la proposition suivante, qui est à la base des travaux de W. Thurston sur les surfaces [Th3] (voir aussi [FLP, exposé 4 p. 59]) :

Proposition 4.9. Soit $\Gamma_{0}$ et $\Gamma_{1}$ deux multi-courbes essentielles sur une surface close. Si $\mathrm{i}\left(\Gamma_{0}, \mathrm{C}\right)=\mathrm{i}\left(\Gamma_{1}, \mathrm{C}\right)$ pour toute courbe fermée simple $\mathrm{C}$, alors $\Gamma_{0}$ et $\Gamma_{1}$ sont isotopes.

D'après un théorème de J. Stallings, tout difféomorphisme de $F \times \mathbf{R}$ dans $\mathrm{F}^{\prime} \times \mathbf{R}$ est isotope à un difféomorphisme produit. La partie b) du théorème 4.5 découle alors directement de la proposition ci-dessus et de l'inégalité de Bennequin semi-locale que voici :

Proposition 4.10. Soit $\xi$ une structure de contact $\mathbf{R}$-invariante et tendue sur le produit $U=\mathrm{F} \times \mathbf{R}$, où $\mathrm{F}$ est une surface close orientée de genre non nul. Soit $\mathrm{C}$ une courbe fermée simple sur $\mathrm{F}=\mathrm{F} \times\{0\}$ et $\Gamma$ une multi-courbe qui scinde $\xi \mathrm{F}$. Pour toute isotopie $\phi_{t}$ de $U$ qui amène $\mathrm{C}$ sur une courbe legendrienne $\phi_{1}(\mathrm{C})$, 
le nombre de tours que fait $\xi$ par rapport au plan tangent à $\phi_{1}(\mathrm{~F})$ le long de $\phi_{1}(\mathrm{C})$ vérifie l'inégalité

$$
\operatorname{deg}\left(\xi, \phi_{1}(\mathrm{~F}) ; \phi_{1}(\mathrm{C})\right) \leq-\frac{1}{2} \mathrm{i}(\Gamma, \mathrm{C}) .
$$

De plus, il existe une isotopie $\phi_{t}$ qui réalise l'égalité.

Démonstration. Quitte à déplacer $\mathrm{C}$ sur $\mathrm{F}$ par une isotopie, on suppose que $\mathrm{C}$ rencontre $\Gamma$ en $i(\Gamma, \mathrm{C})$ points et transversalement. On prend en outre $\xi$ orientable - ce qui revient à passer éventuellement à un revêtement double - et, comme la classe de $\Gamma$ dans $H_{1}(\mathrm{~F} ; \mathbf{Z} / 2 \mathbf{Z})$ est alors nulle, on pose $2 n=\mathrm{i}(\Gamma, \mathrm{C})$.

On observe tout d'abord que l'égalité est atteinte. En effet, on construit facilement un feuilletage $\sigma$ de $\mathrm{F}$ scindé par $\Gamma$ et dans lequel $\mathrm{C}$ est une union de singularités et de feuilles. De plus, le lemme 2.7 fournit une isotopie $\phi_{t}$ de plongements de $\mathrm{F}$ dans $U$ qui amène $\mathrm{F}$ sur une surface $\phi_{1}(\mathrm{~F})$ ayant pour feuilletage caractéristique $\left(\phi_{1}\right)_{*} \sigma$. La courbe $\phi_{1}(\mathrm{C})$ est alors une courbe legendrienne le long de laquelle $\xi$ fait $-\frac{1}{2} \operatorname{Card}(\Gamma \cap \mathrm{C})$ tours par rapport au plan tangent à $\phi_{1}(\mathrm{~F})$.

Soit $\rho: \tilde{\mathrm{F}} \rightarrow \mathrm{F}$ le revêtement associé à $\mathrm{C}$ et $\tilde{\mathrm{C}}$ un relèvement compact de $\mathrm{C}$ dans $\tilde{\mathrm{F}}$. On note $\tilde{\xi}$ le rappel de $\xi$ sur $\tilde{U}=\tilde{\mathrm{F}} \times \mathbf{R}$ et $\tilde{\phi}_{t}, t \in[0,1]$, le relèvement de l'isotopie $\phi_{t}$ à $\tilde{U}$. Le nombre de tours que fait $\xi$ par rapport à $\phi_{1}(\mathrm{~F})$ le long de $\phi_{1}(\mathrm{C})$ est clairement égal au nombre de tours que fait $\tilde{\xi}$ par rapport à $\tilde{\phi}_{1}(\tilde{\mathrm{F}})$ le long de $\tilde{\phi}_{1}(\tilde{\mathrm{C}})$. En outre, les courbes $\tilde{\phi}_{t}(\tilde{\mathrm{C}})$ restent dans un compact de $\tilde{U}$. On paramètre alors $\tilde{\mathrm{F}}$ par $\mathbf{S}^{1} \times \mathbf{R}$ de telle sorte que $\tilde{\mathrm{C}}$ soit la courbe $\mathbf{S}^{1} \times\{0\}$ et on se donne un réel $a>0$ assez grand pour que toutes les courbes $\tilde{\phi}_{t}(\tilde{\mathrm{C}})$ soient contenues dans le domaine $\tilde{U}_{a}=\tilde{\mathrm{F}}_{a} \times \mathbf{R}$, où $\tilde{\mathrm{F}}_{a}=\mathbf{S}^{1} \times[-a, a]$. On établit cidessous l'inégalité voulue en plusieurs étapes. On plonge d'abord $\left(\tilde{U}_{a}, \tilde{\xi}\right)$ dans un modèle abstrait, puis on réalise ce modèle dans la sphère $\mathbf{S}^{3}$ munie de sa structure de contact ordinaire et on conclut à l'aide de l'inégalité de Bennequin classique.

Assertion. Aucune courbe de $\tilde{\Gamma}=\rho^{-1}(\Gamma)$ ne coupe $\tilde{\mathrm{C}}$ en plus d'un point.

Preuve. On suppose qu'une courbe de $\tilde{\Gamma}$ coupe $\tilde{\mathrm{C}}$ en deux points et on note $\mathrm{J}$ un arc de cette courbe joignant deux points d'intersection consécutifs. La composante connexe bornée de $\tilde{\mathrm{F}} \backslash(\tilde{\mathrm{C}} \cup \mathrm{J})$ est un disque $\mathrm{D}$ et $\mathrm{D} \backslash \rho^{-1}(\mathrm{C})$ a au moins une composante connexe $\mathrm{D}_{0}$ dont le bord est l'union de deux arcs, l'un contenu dans $\mathrm{J}$ et l'autre dans $\rho^{-1}(\mathrm{C})$. La restriction de $\rho$ à $\mathrm{D}_{0}$ est alors injective et, en déplaçant $\mathrm{C}$ par isotopie le long de $\rho\left(\mathrm{D}_{0}\right)$, on élimine deux points d'intersection avec $\Gamma$. Ceci est absurde puisque $\operatorname{Card}(\Gamma \cap \mathrm{C})=i(\Gamma, \mathrm{C})$.

L'assertion ci-dessus assure que les composantes de $\tilde{\Gamma}$ qui vont d'un bout à l'autre de $\tilde{\mathrm{F}}$ sont exactement celles qui rencontrent $\tilde{\mathrm{C}}$ et leur nombre est donc égal à $2 n=\mathrm{i}(\Gamma, \mathrm{C})$. Selon que $n$ est nul ou non, il existe alors un plongement incompressible $\psi_{a, n}$ de $\tilde{\mathrm{F}}_{a}$ dans $\mathbf{T}^{2}$ ou dans $\mathbf{S}^{1} \times \mathbf{R}$ tel que

$$
\psi_{a, n}\left(\tilde{\Gamma} \cap \tilde{\mathrm{F}}_{a}\right)=\psi_{a, n}\left(\tilde{\mathrm{F}}_{a}\right) \cap \Gamma_{n},
$$


où

$$
\begin{aligned}
& \Gamma_{0}=\left\{(x, y) \in \mathbf{R}^{2} / \mathbf{Z}^{2} \mid y= \pm 1 / 4\right\} \quad \text { et } \\
& \Gamma_{n}=\{(x, y) \in \mathbf{R} / \mathbf{Z} \times \mathbf{R} \mid n x=0\}, \text { pour } n>0
\end{aligned}
$$

Par suite, si $n=0$ (resp. si $n>0$ ), le lemme 4.2 permet, comme dans la démonstration de la proposition 4.1 , de plonger incompressiblement $\left(\tilde{U}_{a}, \tilde{\xi}\right)$ dans $\left(\mathbf{T}^{2} \times \mathbf{R}, \xi_{0}\right) \quad$ (resp. dans $\left.\left(\mathbf{S}^{1} \times \mathbf{R}^{2}, \xi_{n}\right)\right)$ où $\xi_{n}$ est n'importe quelle structure de contact $\mathbf{R}$-invariante qui imprime sur $\mathbf{T}^{2} \times\{0\}$ (resp. sur $\mathbf{S}^{1} \times \mathbf{R} \times\{0\}$ ) un feuilletage caractéristique scindé par $\Gamma_{n}$.

Soit maintenant $\zeta$ la structure de contact ordinaire sur la sphère $\mathbf{S}^{3} \subset \mathbf{C}^{2}$ et soit $L_{0}$ la courbe legendrienne (non nouée) $\mathbf{S}^{3} \cap \mathbf{R}^{2}$ - dont l'invariant de Thurston-Bennequin $\mathrm{tb}\left(\mathrm{L}_{0}\right)$ vaut -1 .

Le théorème de Darboux permet de paramétrer un voisinage $\mathrm{W}$ de $\mathrm{L}_{0}$ par $\mathbf{S}^{1} \times \mathbf{R}^{2}$ de telle sorte que $\mathrm{L}_{0}$ soit la courbe $\mathbf{S}^{1} \times\{0\}$ et que $\zeta$ ait pour équation $d z+p d \theta=0,(\theta, p, z) \in \mathbf{S}^{1} \times \mathbf{R}^{2}$. Le tore

$$
\mathrm{T}=\left\{(\theta, p, z) \in \mathbf{S}^{1} \times\left.\mathbf{R}^{2}|| p\right|^{2}+|z|^{2}=1\right\}
$$

est convexe car son feuilletage caractéristique $\zeta \mathrm{T}$ est scindé par les deux cercles $\{p= \pm 1\}$. Par suite, $\mathrm{T}$ possède un voisinage homogène isomorphe à $\left(\mathbf{T}^{2} \times \underset{\sim}{\mathbf{R}}, \xi_{0}\right)$. Si $i(\Gamma, \mathrm{C})=0$, on peut donc plonger $\left(\tilde{U}_{a}, \tilde{\xi}\right)$ dans $\left(\mathbf{S}^{3}, \zeta\right)$ en envoyant $\tilde{\phi}_{1}(\tilde{\mathrm{C}})$ sur une courbe legendrienne non nouée $\mathrm{L}$ dont l'invariant de Thurston-Bennequin vaut

$$
\operatorname{tb}(\mathrm{L})=\operatorname{deg}\left(\tilde{\xi}, \phi_{1}(\tilde{\mathrm{F}}) ; \tilde{\phi}_{1}(\tilde{\mathrm{C}})\right)-1
$$

L’inégalité de Bennequin assure alors que le degré est négatif ou nul.

Pour finir, on considère sur $\mathbf{S}^{1} \times \mathbf{R}^{2}$ la structure de contact $\xi_{n}$ d'équation

$$
\cos (2 n \pi x) d y-\sin (2 n \pi x) d t=0
$$

Un calcul direct montre que le plongement de $\mathbf{S}^{1} \times \mathbf{R}^{2}$ dans $\mathbf{S}^{3}$ donné par

$$
(x, y, t) \longmapsto\left\{\begin{array}{l}
\theta=2 \pi x \\
z=\cos (2 n \pi x) y-\sin (2 n \pi x) t \\
p / n=\sin (2 n \pi x) y+\cos (2 n \pi x) t
\end{array}\right.
$$

envoie $\xi_{n}$ sur $\zeta$. Si $\mathrm{i}(\Gamma, \mathrm{C})=2 n>0$, on peut donc plonger, par composition, $\left(\tilde{U}_{a}, \tilde{\xi}\right)$ dans $\left(\mathbf{S}^{3}, \zeta\right)$ en envoyant $\tilde{\phi}_{1}(\tilde{\mathbf{C}})$ sur une courbe legendrienne non nouée L dont l'invariant de Thurston-Bennequin vaut

$$
\operatorname{tb}(\mathrm{L})=\operatorname{deg}\left(\tilde{\xi}, \tilde{\phi}_{1}(\tilde{\mathrm{F}}) ; \tilde{\phi}_{1}(\tilde{\mathrm{C}})\right)+n-1
$$

L’inégalité de Bennequin assure alors que le degré vaut au plus $-n$. 


\section{E. Unicité du cloisonnement}

On démontre ici la partie b) du théorème 4.4. Compte tenu du lemme 4.7, il suffit de prouver que, si les structures de contact $\xi_{0}$ et $\xi_{1}$ sont isotopes, les multicourbes qui les cloisonnent le sont aussi. L'argument est une variante de celui qui conduit à l'inégalité de Bennequin semi-locale. Il repose sur une interprétation appropriée de l'intersection géométrique. Par commodité, on appelle dans la suite indice d'un tore convexe dans une variété de contact tendue le nombre de composantes connexes de toute multi-courbe qui scinde son feuilletage caractéristique.

Lemme 4.11. Soit $\xi$ une structure de contact sur $\mathrm{V}$ cloisonnée par une multicourbe essentielle $\Gamma$ et soit $\mathrm{C}$ une courbe fermée simple de $\mathrm{S}$ dont l'intersection géométrique avec $\Gamma$ n'est pas nulle. L'indice minimal des tores convexes isotopes $\grave{a} \pi^{-1}(\mathrm{C})$ est égal à $\mathrm{i}(\Gamma, \mathrm{C})$ et leur feuilletage caractéristique est scindé par des courbes isotopes aux fibres.

Démonstration. On suppose que $\mathrm{C}$ intersecte $\Gamma$ en $i(\Gamma, \mathrm{C})$ points et on note $\mathrm{F}_{0}$ le tore $\pi^{-1}(\mathrm{C})$. Au-dessus de $\mathrm{C} \backslash \Gamma$ (resp. de $\Gamma \cap \mathrm{C}$ ), le feuilletage $\xi \mathrm{F}_{0}$ est transversal (resp. tangent) aux fibres. En particulier, chaque fibre $\pi^{-1}(q)$, $q \in \Gamma \cap \mathrm{C}$, est une feuille fermée ou une courbe de singularités de $\xi \mathrm{F}_{0}$. Comme $i(\Gamma, C) \neq 0$, le tore $F_{0}$ est convexe et son indice est égal à $i(\Gamma, C)$.

Dans la suite, on désigne par $\rho: \tilde{\mathrm{S}} \rightarrow \mathrm{S}$ le revêtement associé à la courbe $\mathrm{C}$, $\operatorname{par} \tilde{\pi}: \tilde{\mathrm{V}}=\rho^{*} \mathrm{~V} \rightarrow \tilde{\mathrm{S}}$ la fibration induite et par $\tilde{\xi}$ le rappel de $\xi$ sur $\tilde{\mathrm{V}}$. On pose $\tilde{\Gamma}=\rho^{-1}(\Gamma)$ et $\tilde{\mathrm{F}}_{0}=\tilde{\pi}^{-1}(\tilde{\mathrm{C}})$ où $\tilde{\mathrm{C}}$ est un relèvement compact de $\mathrm{C}$ dans $\tilde{\mathrm{S}}$. En outre, on paramètre $\tilde{\mathrm{S}}$ par $\mathbf{R} \times \mathbf{S}^{1}$ de telle sorte que $\tilde{\mathrm{C}}$ soit $\{0\} \times \mathbf{S}^{1}$ et $\tilde{\mathrm{V}}$ par $\mathbf{R} \times \mathbf{T}^{2}$ de telle sorte que $\tilde{\pi}$ soit la projection.

Soit maintenant $\mathrm{F}$ un tore convexe isotope à $\pi^{-1}(\mathrm{C})$ et $\tilde{\mathrm{F}}$ le relèvement compact de $\mathrm{F}$ dans $\tilde{\mathrm{V}}$ obtenu en relevant depuis $\tilde{\mathrm{F}}_{0}$ une isotopie entre $\mathrm{F}_{0}$ et $\mathrm{F}$. Soit encore $a$ et $\varepsilon$ des réels positifs satisfaisant aux conditions suivantes :

- les cercles $\{s\} \times \mathbf{S}^{1}, s \in[-\varepsilon, \varepsilon]$, sont tous transversaux à $\tilde{\Gamma}$ - et coupent donc $\tilde{\Gamma}$ en $\mathrm{i}(\Gamma, \mathrm{C})$ points;

- les cercles $\{ \pm a\} \times \mathbf{S}^{1}$ sont transversaux à $\tilde{\Gamma}$ et le domaine $[-a, a] \times \mathbf{T}^{2} \subset \tilde{\mathrm{V}}$ contient $\tilde{\mathrm{F}}$.

Comme dans la démonstration de la proposition 4.10, les courbes de $\tilde{\Gamma}$ qui vont d'un bord à l'autre de l'anneau $[-a, a] \times \mathbf{S}^{1}$ sont celles qui intersectent $\tilde{\mathrm{C}}$ et sont en nombre $i(\Gamma, C)$. Par suite, il existe un plongement

$$
\psi:[-a, a] \times \mathbf{S}^{1} \longrightarrow[-\varepsilon, \varepsilon] \times \mathbf{S}^{1}
$$

qui est l'identité sur $\{0\} \times \mathbf{S}^{1}$ et vérifie

$$
\psi\left(\tilde{\Gamma} \cap\left([-a, a] \times \mathbf{S}^{1}\right)\right)=\tilde{\Gamma} \cap \psi\left([-a, a] \times \mathbf{S}^{1}\right) .
$$

Le lemme 4.2 fournit alors, comme dans la proposition 4.1, un plongement de contact

$$
\phi:\left([-a, a] \times \mathbf{T}^{2}, \tilde{\xi}\right) \longrightarrow\left([-\varepsilon, \varepsilon] \times \mathbf{T}^{2}, \tilde{\xi}\right)
$$


qui induit l'identité sur $\tilde{\mathrm{F}}_{0}=\{0\} \times \mathbf{T}^{2}$. En outre, l'inégalité de Bennequin semilocale (proposition 4.10) montre que le feuilletage caractéristique d'un tore convexe isotope à $\{0\} \times \mathbf{T}^{2}$ dans $\left([-\varepsilon, \varepsilon] \times \mathbf{T}^{2}, \tilde{\xi}\right)$ est scindé par au moins $i(\Gamma, C)$ courbes qui sont toutes isotopes aux fibres. En particulier, l'indice de $\mathrm{F}$ - qui est égal à celui de $\tilde{\mathrm{F}}$ donc à celui de $\phi(\tilde{\mathrm{F}})$ - vaut au moins $i(\Gamma, \mathrm{C})$.

On complète à présent la démonstration du théorème 4.4-b. Soit $\mathrm{C}$ une courbe fermée simple sur $\mathrm{S}$. Compte tenu de la proposition 4.9 et du lemme 4.11, il suffit de montrer que, si $i\left(\Gamma_{0}, \mathrm{C}\right)$ est nul, $i\left(\Gamma_{1}, \mathrm{C}\right)$ l'est aussi. On suppose donc que $i\left(\Gamma_{0}, C\right)$ vaut 0 et on considère un tore $F_{0}=\pi^{-1}\left(C_{0}\right)$ où $C_{0}$ est une courbe isotope à $\mathrm{C}$ et disjointe de $\Gamma_{0}$. Par construction, le feuilletage $\xi_{0} \mathrm{~F}_{0}$ est transversal aux fibres. Tout tore convexe $\mathrm{F}$ qui s'obtient par une déformation assez petite de $\mathrm{F}_{0}$ a donc un feuilletage caractéristique $\xi_{0} \mathrm{~F}$ scindé par des courbes non isotopes aux fibres. Il résulte alors du lemme 4.11 que, si $i\left(\Gamma_{1}, C\right)$ n'est pas nul, les structures de contact $\xi_{0}$ et $\xi_{1}$ ne sont pas isotopes.

\section{F. Structures virtuellement vrillées}

On termine cet exposé par un résultat de finitude pour les structures de contact virtuellement vrillées.

Théorème 4.12. Soit V une variété connexe et orientée, fibrée en cercles audessus d'une surface close $\mathrm{S}$. Les structures de contact orientables et virtuellement vrillées sur $\mathrm{V}$ forment un nombre fini de classes d'isotopie borné par

$$
\left\{\begin{aligned}
\sup \{0,-\chi(\mathrm{S})-\chi(\mathrm{V}, \mathrm{S})-1\} & \text { si } \chi(\mathrm{V}, \mathrm{S}) \leq 0, \\
1+\sup \{0,-\chi(\mathrm{S})-\chi(\mathrm{V}, \mathrm{S})-1\} & \text { si } \chi(\mathrm{V}, \mathrm{S})>0 .
\end{aligned}\right.
$$

En fait, avec les formes normales dégagées dans [Gi4], on obtient une description précise de tous les exemples potentiels de (classes d'isotopie de) structures de contact virtuellement vrillées sur V. Il est par ailleurs probable que toutes les structures de contact ainsi décrites sont effectivement tendues - même holomorphiquement remplissables - et que les techniques de chirurgie développées par R. Gompf dans [Go] permettraient de le prouver. Du reste, la proposition 2.13 montre, par des astuces de revêtements, l'existence de structures de contact virtuellement vrillées sur les variétés fibrées en cercles au-dessus du tore et dont le nombre d'Euler est inférieur ou égal à -2 .

Lemme 4.13. Si V porte une structure de contact virtuellement vrillée et d'enroulement positif ou nul, celle-ci est isotope à une structure cloisonnée par une courbe connexe contractile et $\chi(\mathrm{V}, \mathrm{S})$ est strictement positif.

Démonstration. Soit $\mathrm{A} \subset \mathrm{S}$ un anneau incompressible, $\mathrm{R}$ la surface $\mathrm{S} \backslash$ Int A et $\mathrm{W}$ le tore épais $\pi^{-1}(\mathrm{~A})$. D'après le lemme 4.6, toute structure de contact tendue d'enroulement positif ou nul est isotope à une structure $\xi$ qui, au-dessus de $\mathrm{R}$, 
est cloisonnée par un système d'arcs $\Gamma_{\mathrm{R}}$. La restriction de $\xi$ à $\mathrm{W} \simeq \mathbf{T}^{2} \times[0,1]$ est ainsi une structure de contact tendue qui trace sur chaque composante de $\partial \mathrm{W}$ un feuilletage ayant des feuilles fermées ou des cercles de singularités parallèles aux fibres. Vu ce comportement au bord, le théorème 1.5 de [Gi4] assure que $\left.\xi\right|_{\mathrm{w}}$ est universellement tendue et est isotope, relativement à $\partial \mathrm{W}$, à une structure de contact $\eta$ cloisonnée par un système d'arcs $\Gamma_{\mathrm{A}}$. Ainsi $\xi$ est isotope à une structure de contact cloisonnée par la multi-courbe $\Gamma=\Gamma_{\mathrm{R}} \cup \Gamma_{\mathrm{A}}$.

Pour conclure, on se donne sur $\mathrm{V}$ une action libre du cercle dont les orbites sont les fibres de $\pi$. D'après le lemme $4.7, \xi$ est isotope à « la $»$ structure invariante cloisonnée par $\Gamma$. Comme $\xi$ est tendue mais pas universellement, la proposition 4.1 montre que $\Gamma$ est une courbe connexe contractile et que $\chi(\mathrm{V}, \mathrm{S})>0$.

Démonstration du théorème 4.12. D'après les lemmes 4.13, 4.7 et la proposition 4.1, les structures de contact virtuellement vrillées et d'enroulement positif ou nul forment au plus une classe d'isotopie, et aucune si $\chi(\mathrm{V}, \mathrm{S}) \leq 0$. On étudie donc désormais les structures de contact virtuellement vrillées et d'enroulement strictement négatif.

Soit $\mathrm{D} \subset \mathrm{S}$ un disque, $\mathrm{R}$ la surface $\mathrm{S} \backslash \operatorname{Int} \mathrm{D}$ et $\mathrm{W}$ le tore plein $\pi^{-1}(\mathrm{D})$. Comme d'habitude, on paramètre $\mathrm{W}$ par $\mathbf{D}^{2} \times \mathbf{S}^{1}$ de telle sorte que $\left.\pi\right|_{\mathrm{W}}$ soit la projection sur $\mathbf{D}^{2}$. D'après le lemme 2.8 , toute structure de contact d'enroulement $-n, n>0$, est isotope à une structure $\xi$ pour laquelle les fibres au-dessus de $\mathrm{R}$ sont legendriennes et d'enroulement $-n$. Dans ces conditions, $\partial \mathrm{W}$ est un tore convexe d'indice 2 (lemme 2.11) et les singularités de son feuilletage caractéristique $\xi \partial \mathrm{W}$ forment des courbes de classe $(n, n \chi(\mathrm{V}, \mathrm{S})+\chi(\mathrm{S})-1)$ dans $H_{1}(\partial \mathrm{W} ; \mathbf{Z}) \cong \mathbf{Z}^{2}$ (corollaire 3.5$)$.

Assertion. Ou bien $n \chi(\mathrm{V}, \mathrm{S})=-\chi(\mathrm{S})$, ou bien $n=1$ et $\chi(\mathrm{V}, \mathrm{S})<-\chi(\mathrm{S})$. Preuve. Pour tout $a \in] 0,1]$, on note $\mathrm{T}_{a}$ le tore $a \mathbf{S}^{1} \times \mathbf{S}^{1}$. D'après la proposition 3.22 de [Gi4], la restriction de $\xi$ à $W$ est isotope, relativement au bord, à une structure de contact $\eta$ transversale à $\{0\} \times \mathbf{S}^{1}$ et dont les feuilletages caractéristiques $\eta \mathrm{T}_{a}$ ont les propriétés suivantes :

- $\eta \mathrm{T}_{a}$ est une suspension sauf pour un nombre fini de valeurs $a_{1}, \ldots, a_{k} \in$ ] $0,1]$;

- $\eta \mathrm{T}_{a_{i}}, 1 \leq i \leq k$, n'a aucune orbite fermée et ses singularités forment deux cercles.

Chaque feuilletage $\eta \mathrm{T}_{a}$ détermine alors une droite $\delta_{a}$ dans $\mathbf{R}^{2} \cong H_{1}\left(\mathrm{~T}_{a}, \mathbf{R}\right)$ qui, pour $a \notin\left\{a_{i}\right\}$, porte les cycles asymptotiques et, pour $a \in\left\{a_{i}\right\}$, contient la classe des cercles de singularités. Cette droite varie continûment avec $a$ et converge vers $\delta_{0}=\mathbf{R} \times\{0\}$ quand $a$ tend vers 0 . Les droites $\delta_{a}, a \in[0,1]$, décrivent donc un connexe $\Delta$ de $\mathbf{P}^{1}(\mathbf{R})$, connexe qui ne contient pas la droite $\{0\} \times \mathbf{R}$ car l'enroulement de $\eta$ est strictement négatif. Ainsi, $\Delta$ est l'intervalle $\left[\delta_{1}, \delta_{0}\right]$ pour l'orientation naturelle de $\mathbf{P}^{1}(\mathbf{R})$ et $n \chi(\mathrm{V}, \mathrm{S})+\chi(\mathrm{S}) \leq 0$ puisque $\delta_{1}$ est dirigée par $(n, n \chi(\mathrm{V}, \mathrm{S})+\chi(\mathrm{S})-1)$. D'autre part, pour toute droite rationnelle $\delta \in\left[\delta_{1}, \delta_{0}[\right.$, on peut trouver un $\left.a \in] 0,1\right]$ tel que $\delta_{a}$ soit égale à $\delta$ et 
que $\mathrm{T}_{a}$ soit un tore convexe d'indice 2 . En fait, pour cela, il faut éventuellement perturber $\eta$ par une petite isotopie qui ne détruit pas les propriétés utiles.

À partir de ces observations, la preuve est identique à celle de l'assertion similaire dans la démonstration de la proposition 3.7.

Pour terminer la démonstration, on utilise la classification des structures de contact sur le tore plein établie dans [Gi4]. Si $n \chi(\mathrm{V}, \mathrm{S})=-\chi(\mathrm{S})$, la droite $\delta_{1}$ est dirigée par le vecteur $(n,-1)$. Avec cette condition au bord, le théorème 1.6 de [Gi4] affirme que la restriction de $\xi$ à W est universellement tendue. On se trouve du coup dans la situation de la section 3.C : $\xi$ est isotope à une structure de contact tangente aux fibres et n'est pas virtuellement vrillée. Si $n=1$ et si $\chi(\mathrm{V}, \mathrm{S})<-\chi(\mathrm{S})$, la droite $\delta_{1}$ est dirigée par le vecteur $(1,-m)$, où $m=1-\chi(\mathrm{V}, \mathrm{S})-\chi(\mathrm{S})>1$. Le théorème 1.6 de [Gi4] dit alors qu'il y a sur $\mathrm{W}$, à isotopie relative au bord près, $m-1$ structures de contact tendues qui coïncident avec $\xi$ sur $\partial \mathrm{W}$, dont une (seule) est universellement tendue. En outre, l'existence sur $\mathrm{V}$ d'une structure de contact universellement tendue et d'enroulement -1 assure que, si $\left.\xi\right|_{\mathrm{W}}$ est universellement tendue, $\xi$ l'est aussi. On obtient ainsi les bornes annoncées.

Remarque. La démonstration ci-dessus fait apparaître que -1 est la seule valeur strictement négative possible pour l'enroulement d'une structure de contact virtuellement vrillée.

Par ailleurs, les résultats de cette partie permettent de compléter quelque peu l'énoncé du théorème 2.3 : si une structure de contact $\xi$ sur $\mathrm{V}$ est d'enroulement positif ou nul, il existe non seulement une courbe legendrienne isotope à la fibre et d'enroulement nul mais tout un tore transversal à $\xi$ et dont les caractéristiques sont isotopes aux fibres. Ce tore est la version de contact de la feuille compacte trouvée par W. Thurston [Th2]. Lorsque $\xi$ est tendue, son existence résulte soit $\mathrm{du}$ théorème 4.4, soit du lemme 4.13. Lorsque $\xi$ est vrillée, un théorème de Y. Eliashberg [El1] assure qu'une modification de Lutz le long d'une fibre produit une structure de contact isotope à $\xi$. Or une telle modification fait clairement apparaître le tore cherché (voir par exemple [Gi2]).

\section{Références}

[Al] S. Altschuler, A geometric heat flow for one-forms on three-dimensional manifolds, Illinois J. Math. 39 (1995), 98-118.

[Be] D. Bennequin, Entrelacements et équations de Pfaff, Astérisque 107-108, Soc. Math. France 1983, 83-161.

[Co] V. Colin, Recollement de variétés de contact tendues, Bull. Soc. Math. France 127 (1999), 43-69.

[EHN] D. Eisenbud, U. Hirsch, W. Neumann, Transverse foliations of Seifert bundles and selfhomeomorphisms of the circle, Comment. Math. Helv. 56 (1981), 638-660.

[El1] Y. Eliashberg, Classification of over-twisted contact structures on 3 -manifolds, Invent. Math. 98 (1989), 623-637.

[El2] Y. Eliashberg, Contact 3 -manifolds, twenty years since J. Martinet's work, Ann. Inst. Fourier 42 (1992), 165-192. 
[El3] Y. Eliashberg, Filling by holomorphic discs and its applications, London Math. Soc. Lect. Note Ser. 151, Cambridge Univ. Press 1991, 45-67.

[ET] Y. Eliashberg, W. Thurston, Confoliations, Univ. Lecture Ser. 13, Amer. Math. Soc. 1998.

[FLP] A. Fathi, F. Laudenbach, V. Poenaru, Travaux de Thurston sur les Surfaces, Astérisque 66-67, Soc. Math. France 1991 (seconde édition).

[Gh1] É. Ghys, Classe d'Euler et minimal exceptionnel, Topology 26 (1987), 93-105.

[Gh2] É. Ghys, Rigidité différentiable des groupes fuchsiens, Inst. Hautes Études Sci., Publ. Math. 78 (1993), 163-185.

[Gi1] E. Giroux, Convexité en topologie de contact, Comment. Math. Helv. 66 (1991), 637-677.

[Gi2] E. Giroux, Topologie de contact en dimension 3 [autour des travaux de Ya. Eliashberg], Astérisque 216, Soc. Math. France 1993, 7-33.

[Gi3] E. Giroux, Une infinité de structures de contact tendues sur une infinité de variétés, Invent. Math. 135 (1999), 789-802.

[Gi4] E. Giroux, Structures de contact en dimension trois et bifurcations des feuilletages de surfaces, Invent. Math. 141 (2000), 615-689.

[Go] R. Gompf, Handlebody construction of Stein surfaces, Ann. of Math. 148 (1998), 619693.

[Ka] Y. Kanda, Classification of tight contact structures on the 3 -torus, Comm. Anal. Geom. 5 (1997), 413-438.

[Le] G. Levitt, Feuilletages des variétés de dimension 3 qui sont des fibrés en cercles, Comment. Math. Helv. 53 (1978), 572-594.

[Lu] R. Lutz, Structures de contact sur les fibrés principaux en cercles de dimension 3, Ann. Inst. Fourier 27 (1977), 1-15.

[Ma] S. Matsumoto, Some remarks on foliated $\mathbf{S}^{1}$-bundles, Invent. Math. 90 (1987), 343-358.

[Mi] J. Milnor, On the existence of a connection with curvature 0, Comment. Math. Helv. 32 (1958), 215-223.

[Ro] R. Roussarie, Plongements dans les variétés feuilletées et classification de feuilletages sans holonomie, Inst. Hautes Études Sci., Publ. Math. 43 (1974), 101-141.

[ST] A. Sato, T. Tsuboi, Contact structures of closed 3 -manifolds fibered by the circle, prépublication (Meiji University), 1994.

[Th1] W. Thurston, Noncobordant foliations of $\mathbf{S}^{3}$, Bull. Amer. Math. Soc. 78 (1972), 511514.

[Th2] W. Thurston, Foliations of 3 -manifolds which are circle-bundles, thèse (UC-Berkeley), 1972.

[Th3] W. Thurston, On the geometry and dynamics of diffeomorphisms of surfaces, Bull. Amer. Math. Soc. 19 (1988), 417-431.

[Th4] W. Thurston, Contact structures and foliations on 3 -manifolds, exposé oral, West Coast Topology Conf. (Stanford), 1992.

[Wa] F. Waldhausen, On irreducible 3 -manifolds which are sufficiently large, Ann. of Math. 87 (1968), 56-88.

[Wo] J. Wood, Bundles with totally disconnected structure group, Comment. Math. Helv. 46 (1971), 257-273.

Emmanuel Giroux

Unité de Mathématiques Pures et Appliquées

École Normale Supérieure de Lyon

46, allée d'Italie

F-69364 Lyon cedex 07

France

e-mail: Emmanuel.Giroux@umpa.ens-lyon.fr

(Received: October 29, 1999) 